\title{
Digital Intimacies
}

\section{Doing Digital Media Differently}

\author{
Helga Sadowski
}

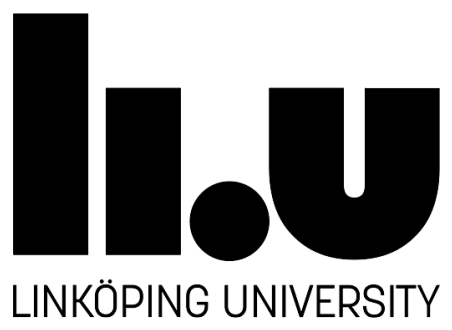

Linköping Studies in Arts and Science No. 691

Faculty of Arts and Sciences

Linköping 2016 


\section{Linköping Studies in Arts and Science • No. 691}

At the Faculty of Arts and Sciences at Linköping University, research and doctoral studies are carried out within broad problem areas. Research is organized in interdisciplinary research environments and doctoral studies mainly in graduate schools. Jointly, they publish the series Linköping Studies in Arts and Science. This thesis comes from Unit of Gender Studies at the Department of Thematic Studies.

Distributed by:

Department of Thematic Studies

Linköping University

58183 Linköping

Helga Sadowski

Digital Intimacies

Doing Digital Media Differently

Edition 1:1

ISBN 978-91-7685-718-2

ISSN 0282-9800

(c) Helga Sadowski

Department of Thematic Studies 2016

Covert art: Helga Sadowski

Artwork chapter title pages: Sarah Hornikel \& Carmen Geduhn

Printed by: LiU-Tryck, Linköping 2016 




\section{Acknowledgements}

The past years as a PhD student have been very insightful, eventful and exciting, and I am very thankful to all the people that affected me, this thesis and its formation process in uncountable, most versatile and intimate ways.

Firstly, my deepest gratitude goes out to my amazing supervisors Cecilia Åsberg and Jenny Sundén. Thank you Cissi for your optimism, for always believing in me and for probably being my biggest cheerleader. That helped a lot. Thank you for helping me to develop my project in this friendly feminist setting of unconventional and interdisciplinary (post-) humanities. Thank you for all the creative input, for the laughs, and for introducing me to so many helpful and mind-bending academic texts (No worries, I will return the pile of books that you lent me soon ;-). Thank you also for occasionally taking me out of my comfort zone, which for example made me find myself networking at the other end of the world. Every time you (gently) pushed me out of that comfort zone, it proved to be incredibly useful for me and the development of my work.

Thank you so much for all your help and support, Jenny. I am super impressed by your ability to somehow know what I want to say when I am still struggling to find the words or to organize my thoughts. Your feedback has been indispensable and always inspiring. Thank you also for not minding (too much I hope) feeling like a broken record, when you had to explain certain things to me multiple times ;-). I always enjoyed visiting you at Södertörn Högskola a lot, or when we met up in one of Stockholm's Cafés. I always felt more hopeful afterwards.

I also would like to thank Jeff Hearn who accompanied me in very early stages of the $\mathrm{PhD}$ process, when my project was very different from what it is now. Thank you for your dedication and kind support, for 'showing me the academic ropes', and for your great humor.

Thanks also to the fantastic five, which are my co-PhD students starting also in September 2011. I am in awe when I see your incredible 
transformations into the brilliant and passionate scholars that you are, Tara Mehrabi, Line Henriksen, Marie-Louise Holm, Marietta Radomska and Desireé Ljungcrantz. Thank you for all your encouraging words, PhD student survival tips, cozy dinners and occasional craziness. You are definitely ready to take over the world.

Warm thanks go also out to all who have participated in my 60\% seminar and final seminar, and offered thoughtful and enlightening comments. Thank you Jette Koefoed for providing much needed advice in how to structure my text at a point when it was still rather messy. Thank you Lissa Holloway-Attaway for engaging with my text in such a thorough manner. Thank you also Anna Lundberg for acting as a discussant for my dissertation back then and now, and for pointing out the appearances of irony in some of my material as well as their entanglement with neoliberal market logics. Thank you also Bodil Axelsson for your insightful comments, for welcoming me so warmly during my brief, but intense, interludes at and with Tema Q und ACSIS, and enabling and facilitating the exchange with the ICS at the University at Western Sydney. Thank you also Karin Fast for your extensive and considerate comments during and after the final seminar, and for the amazing course on mediatization processes. A big thanks also to the PhD student discussants at those seminars, Lisa "Simpson" Lindén and Marietta Radomska, you are walking encyclopedias of awesome knowledges to me.

My gratitude also goes out to all who acted as (more or less) voluntary proofreaders of my text during different stages of messiness. Thank you Carmen Geduhn, Daniel Sadowski, Jami Weinstein, Hanne Fjelde and Reinert Huseby Karlsen for fighting fearlessly through the chaos and offering thoughtful advice on how to get a grip on it. Thanks go also out to Sarah Hornikel and Carmen Geduhn for the artwork on the separation pages (I love it!), and to Liz Sourbut for the English language check and working 'outside the office hours' in order to help me meeting the deadline.

I am grateful for all my wonderful colleagues who made and make Tema Genus such an extraordinary place and for all the things that you taught me over the years. Thank you Alma Persson (also for you help 
with the Swedish abstract), Alp Biricik, Anna Wahl, Anne-Charlott Callerstig, Anne-Li Lindgren, Åsa-Karin Engstrand, Berit Starkman, Björn Pernrud, Dag Balkmar, Edyta Just, Elisabeth Samuelsson, Emma Strollo, Frida Beckman, Jami Weinstein, Justin Makii, Katherine Harrison, Klara Goedecke, Linn Sandberg, Lotta Callerstig, Madina Tlostanova, Magda Górska, Malena Gustavson, Margrit Shildrick, Marianna Szcyzgielska, Monica Obreja, Nina Lykke, Olga Cielemęcka, Pat Treusch, Pia Laskar, Redi Koobak, Roger Klinth, Silje Lundgren, Stina Backman, Tanja Joelsson, Tanya Bureychak, Ulrica Engdahl, Victoria Kawesa, Wera Grahn, Wibke Straube, and others in various degrees of attachment to the Tema $\mathrm{G}$ universe.

Thank you also Ian Dickson, Eva Danielson, Carin Ennergård, Barbro Axelsson, Camilla Jungström Hammar, Micke Brandt, Beatrice Rågard, and Anne-Christine Lindvall for technical and administrative support, and your patience with me.

Big thanks also go out to all involved at the Posthumanities hub for all the though-provoking discussions (Thank you Cissi and all visiting scholars!), and to Iris van der Tuin and all involved in the on 'How Matter Comes to Matter' COST action. In particular, the 'New Materialism Embracing the Creative Arts' work group and Marie-Luise Angerer who facilitated my short research stay at the Academy of Media Arts Cologne which influenced my take on affect and ASMR.

Above this, I would also like to acknowledge some people who constantly have backed me up and reminded me of the pleasurable sides of life during a sometimes bumpy PhD ride: Thank you Annekatrin, Colin, Hanne, Holly, Ida, JM, Josefin and Magnus for unforgettable Midsommar celebrations, wild Thanksgivings, and long-lasting 'Afterworks'. You made me grown attached to Uppsala. Thank you Agnes, Bea, Diana, Marc, Martin and the rest of the crew for the Sarek trek, dinner parties in the company of many quadrupled friends, and for carrying boxes up and down the stairs every time we moved. Thanks also to the Huseby Karlsens for always welcoming me so warmly to their home and for the wonderful cross-country skiing excursions including barbequing in the snow. 
Thank you also Tove, Veronica, Elisabeth, Camilla, and Carin: It still brings back a smile to my face when I think of our Oxford adventures and the puffins. Thank you also to Mina, Måns and Tintin for being such great short-term flatmates.

My deepest gratitude furthermore goes out to my dearest friends

Carm, Tine, Julia, Doro, Katha, Nadine and Stefan: you will always be my role models for life. I admire you for being so strong, independent and groovy at the same time, and I will forever be grateful that I can be a part of your lives. You will be always in my heart. I am sending out big love also to my Amsterdam/Utrecht crowd: Kathrin, Olli, Nina, Robin, Fidel, Marlenchen: Hanging out with you on Nootje or at Roest are my idea of 'a happy place'. I miss you very much!

I thank my parents and brother for unconditional love and support all my life. It means everything to me!

Last but not least I want to thank my partner Reinert. I could not have done this without you. You made the past nine years the best of my life and I am looking so much forward to the adventures to come. Everything is better when you are around.

Linköping, $3^{\text {rd }}$ November 2016 Helga Sadowski 



\section{Table of Contents}

1 Introduction: Digital Intimacies in Everyday Internet

Cultures ................................................................. 15

1.1 Aims and Research Questions ................................................ 18

1.2 Hate and Hostility Online ............................................................23

Hatr: Countering Online Hate ...............................................2 27

1.3 Women in Tech 2.0 ...............................................................28

Women*-Centered Coding Initiatives .....................................30

1.4 The Whispering Women of ASMR ............................................32

On Whispering and Gazing......................................................34

1.5 Digital Inimicalities and Doing Digital Media Differently .......35

Outline of the Book .................................................................... 37

2 Theories of Intimacy .............................................. 41

What's in a Kiss? ................................................................... 41

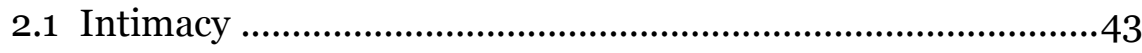

Public Intimacy ....................................................................46

Affect and Intimacy...........................................................49

Remediating Intimacy ....................................................... 52

Intimacy and Being Digital .............................................58

Digital Intimacy...................................................................62

3 Approaching Digital Intimacy: Methods and Materials .............................................................. 67

3.1 Situating the Thesis Epistemologically ..................................67

Departures: How I Encountered my Material ....................... 71 
3.2 Methodological Considerations: Remixing with an Ethnographic Attitude .74

Generate, Play, Borrow, Move, and Interrogate ...................83

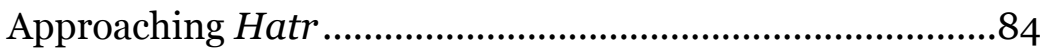

Approaching Women's Coding Initiatives ...........................85

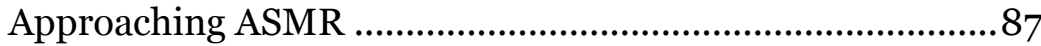

4 Hatr.org: An Initiative against Online Hate..............93

4.1 Background: Feminist Digital Activism as Cultural

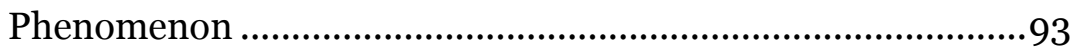

Introducing Hatr ...............................................................98

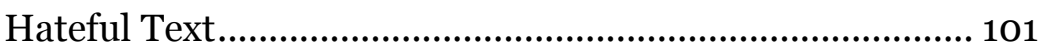

4.2 Affective Economies of Hate..................................................104

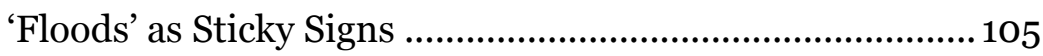

Other Stickers..................................................................... 107

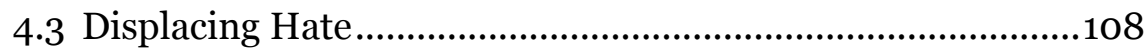

Relaying Expulsion .............................................................109

Reacting to Hate...................................................................112

4.4 Generating a Digital Testimony..............................................112

Monetizing the Hate ...........................................................114

4.5 Discussion: Hatr's Strategies as Feminist Activist Tool..........116

5 Getting Intimate with Code: Coding as an On/Off Love

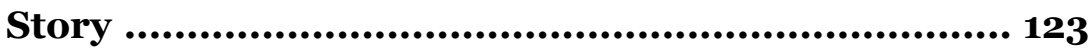

5.1 CodeGirl ............................................................................. 123

Emotionality as Thematic Concept .................................... 124

5.2 An On/Off Love Story with Coding …...................................... 128

Going Back and Forth ........................................................ 134

5.3 Women*-Centered Coding Groups as an Approach to Revive a Love Story 
5.4 Soft Code Skills ....................................................................... 138

Before the Workshop ........................................................140

5.5 The HackerWomen's Workshop .............................................. 142

Work Life Lunch Talk ....................................................... 144

Work as Affective Impasse................................................ 146

5.6 Discussion: Coding, the Soft Way?.......................................... 149

6 Friendly Faces in Digital Places: Exploring the World of ASMR Videos.....................................................157

6.1 What is ASMR? ...................................................................... 157

Meeting Heather Feather...................................................... 163

Watching ASMR ................................................................ 165

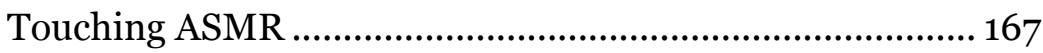

6.2 Twisting Representations in ASMR …................................ 172

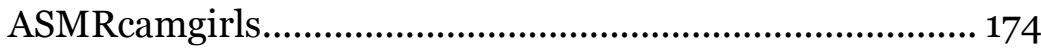

Intimate Complicity .........................................................177

6.3 ASMR and the Science of Sleep …..........................................180

6.4 Discussion: A new Genre of Intimacy? .................................. 184

7 Conclusion: Countering Digital Inimicalities ......... 189

Remixing Digital Intimacies for a Feminist Internet

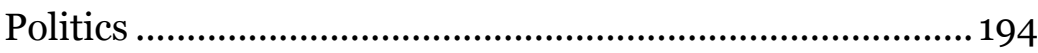

Remediating Older Forms of Feminist Activism ................ 195

Digital Intimacy and the Neoliberal Context - Money Can’t Buy Me Love ....................................................................... 196

Embracing Digital Intimacy ............................................... 199

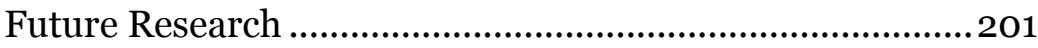

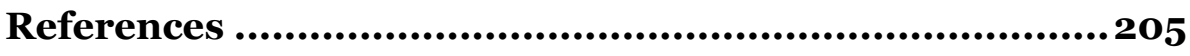


INTRODUCTION:

DIGITAL INTIMACIES

IN EVERYDAY

INTERNET CULTURES 



\title{
1 Introduction: Digital Intimacies in Everyday Internet Cultures
}

\author{
${ }^{66}$ You are all just ugly! \\ (Posted in the comment section of an unknown homepage, \\ and re-posted on the anti-trolling platform hatr.org) ${ }^{1}$
}

${ }^{66}$ Railsbridge is working to make tech more diverse and welcoming by teaching programming, connecting human beings, and listening to people's needs.

(Homepage of an organization that aims to bring more diversity into tech) ${ }^{2}$

${ }^{66}$ And...they are very soft. It feels very nice...against your skin. Do you agree?"

(YouTube starlet VeniVidiVulpes asks the viewer of her ASMR video clip after 'touching' them with a brush)3

${ }^{1}$ This translation from German to English is mine. http://hatr.org/archive/4, last accessed 24/10/2016.

${ }^{2} \mathrm{http}: / /$ railsbridge.org/, last accessed 14/10/2016.

3 https://www.youtube.com/watch?v=m-lHoJzlrJU, last accessed 14/10/2016. 
What it means to be online and to live with digital technologies has changed tremendously over the last couple of decades. Processes of digitalization 4 have crept into even the most mundane arenas of everyday life: From work meetings on Skype, to touch displays on train ticket machines, to mobile phones replacing banking infrastructures in Africa, India and Eastern Europe, most people on earth have been affected by the ever-changing forms of digital communication in one way or another.

Communicating digitally no longer means being tied to stationary computers. Devices such as laptops and mobile phones have become intimate life companions. Laptops are taken to bed, mobile phones are held close to the heart when a right-handed person is swiping through profiles on tinder, sweaty streaks on the tablet testify to states of being really in touch with the touchscreen.

The content and ways of communicating have also become more intimate: "People who have never done so before are telling personal stories through digital forms, storing and exchanging those stories in sites and networks that would not exist without the world wide web", writes media and communication sociologist Nick Couldry (2008, 374). Intimate pictures, (snap-)chats, and videos are shared and passed around in schoolyards to a degree that leads Sherry Turkle, an expert on writing about people's relationships with computers, to conclude that there is a reinvention of intimacy taking place in which the public

4 There are different definitions of the term 'digitalization': Manuel Castells regards it as a key characteristic of modern times and emphasizes how underlying media and communication systems can unlock understanding for many, or even most, characteristics of contemporary life (2010). Other analyses focus on the interplay of digitalization and economic globalization and how this influences conceptions of all aspects of society, be it culture, finance or the social (cf. Sassen 1998). Anne Kaun and Karin Fast see it as part of a broader processes of mediatization, which "encompasses all processes of change that are media induced or that are related to a change in the media landscape over time", a process which "includes changes in the media ecology that are linked to other large-scale social changes" $(2014,12)$. It is precisely this connection between changes in mediated cultures and large-scale social changes that I am referring to when using the term 'digitalization' in the following. 
sharing of experiences can be a deliberate movement towards intimacy (Turkle 2011, 175).

In other words, the internet and digital media have become established as playgrounds for intimate relations, and intimacy is probably much more often and much more systematically created via these media than romantic imaginaries would have us believe - no matter how hard many want to see the notion of 'inner touch' as un-mediated (Meßmer, Schmidbaur, and Villa 2014). Through processes of digitalization, contact zones of the digital and the intimate, or affectspheres, have multiplied and diversified. This does not only mean a shift in the distinction between the public and the private, but also an increase in potential vulnerabilities. This doctoral dissertation explores three different digital cultures and initiatives that can be seen as facets of how the digital is becoming more intimate, and of how intimacy is changing in the context of digitalization.

My first strategic example concerns a topic that has become notorious in recent years; namely, online hate speech. People being attacked at the most intimate levels is a "rising global phenomenon" (Gardiner et al. 2016). I chose to analyze an initiative called hatr.org that seeks to interrupt the circle of hate and how it travels online.

Secondly, I look at women-centered coding initiatives that try to re-brand coding and computing as more approachable, social, and passionate and, in this way, seek to establish an intimacy with coding that is perceived to be missing between women and digital technologies.

Lastly, I analyze aspects of the YouTube subcultures ASMR (Autonomous Sensory Meridian Response), in which intimate situations are recreated for the camera in order to give viewers pleasurable bodily

\footnotetext{
5 Subcultures as defined by Dick Hebdige (1979) initially described a group of people who shared a common ideology or interest, which ususally was in opposition to the parent culture. Subcultures made their identity visible by applying a certain (life)style (e.g. punks). More recent work suggest that subcultures now form primarily based on common taste and interest, and less on resistance and opposition to mass culture (Hartley et al. 2002; Thornton 1995). I see the subculture around ASMR in the second vein.
} 
reactions; for instance, when the performer pretends to touch the viewer through the screen (as in the example above).

While these three strategic examples, chosen as snapshots of contemporary digital cultures, are very different from each other, I argue that they are symptomatic of what I will define as digital intimacy. The diversity of the cases furthermore points out the fact that digital intimacy needs to open up prevailing conceptions of intimacy for the digital context.

What unites the three cases - besides the fact that they use, transform, and extend notions of intimacy in digital contexts - is that, as I will show, they aim to 'do digital media differently' in the sense that they can be seen as trying to counteract particular inimicalities of digital cultures. What I mean by this is that there has been a lot of negative news about what it means "to be a woman online" (Vagianos 2014) in recent years. Questions of visual representation, hate speech, or low levels of participation in tech businesses have been transferred into a web 2.o context, and have affected not only women but also other/intersecting groups, such as feminist activists, members of LGBTQI communities, or people of color, to severe extents.

\subsection{Aims and Research Questions}

In this doctoral dissertation, I attempt to explore how the initiatives briefly quoted above, namely the hatr.org project, diverse coding initiatives that focus on women, and actors within the ASMR subculture, try to counteract the inimicalities that arise in the digital sphere, sometimes in a straightforward manner, sometimes rather playfully.

I also want to investigate whether (digital) intimacy itself can be theorized as a strategic tool for feminist internet politics. My intention is thus to explore such initiatives and discuss how they can be utilized, by critically evaluating their projects, strategies, and phenomena, which might be seen as counteracting digital inimicalities. I believe that 
there is a need for this work because, despite widespread feminist writing and activism online, there are few joint strategies to counteract inimicalities and hostility in post-cyberfeminist times.

I engage in this research because I understand such inimicalities to be devastating, discouraging, and hurtful to women and other targeted and disadvantaged groups. In that sense, this dissertation is also about the 'women and technology' question, ${ }^{6}$ carried forward into the era of digitalization and social media.

By analyzing these three different women-centered/feminist initiatives, which engage with digital media in alternative ways, this thesis contributes to feminist cultural studies of new media by discussing the role of digital intimacies in feminist internet politics. I am looking for constructive solutions to the question of how to face particular problems that, from my point of view, plague contemporary online cultures at the intersections of gender, race, sexuality, ability, and other identity markers.

I thus have two main aims in writing this dissertation: One aim is theoretical, the other more analytical/political. The more theoretical aim, which is a step on the way to being able to contribute to the femi-

${ }^{6}$ The 'technology question in feminism' is an ongoing debate that resonates with and is a continuation of questions of gender and science (Harding 1986). This is because both, science and technology, are domains from which women historically have structurally been excluded. As a consequence, not only science but also technology became men's monopolies and were identified by early feminists as an excluding source of power (Wajcman 2001, 5976). Within a 'technology as culture' approach, feminist scholars such as Judy Wajcman have explored how the relationship of men and masculinities with technology (starting with military and industrial machinery) became naturalized and often rooted in biological determinism, while women's contributions to the history of technology were silenced (Wajcman 2010, 144). Wajcman furthermore ascribes a particular role to the rise of mechanical and civil engineering at the end of the 19th century in the manifestation of the masculinity-technology link. She argues that these professions were constructed as male-white and "characterised by the cultivation of bodily prowess and individual achievement", while "femininity was being reinterpreted as incompatible with technological pursuits" (ibid.). It was this development, she argues, that led to the modern understanding of technology as a masculine domain which still influences technocultures today. 
nist analytical toolbox, is to explore how contemporary everyday internet and digital media practices mediate intimacy in ways that complicate perceptions of 'digital' and 'intimacy' as incompatible or mutually exclusive realms. My interest in intimacy also means underlining the dimension of the body, and by doing so building a counterpart to disembodied, cold, neutral, and impersonal cybernarratives, which tend to "obviate the body at the keyboard" and silence "once again the very question of embodiment" (Gillis 2004, 190).

The analytical/political aim is to better understand the exclusionary and inimical aspects of digital cultures and to provide strategies for a feminist toolbox to counteract those inimicalities. I examine what counter-initiatives I can find in the anti-online harassment initiative hatr.org, in coding courses which are conceptualized 'against the grain', and the quirky subculture of ASMR. I am curious about why digital media are taken up and used in these particular ways here and how theorizing digital intimacy can be used to understand this in more depth. This curiosity has helped me to explore the three different cases.

Following my dual focus on theoretical consideration and analytical/political contributions, my main research questions are:

- How are notions of intimacy transformed and remediated in contemporary digital media cultures?

- Can digitally intimate initiatives and approaches confront and counteract the inimical aspects of everyday communication cultures, and if so, how?

- In what ways can digital intimacy be conceptualized as a tool for a feminist internet politics?

The first question has an overarching theoretical function. It is important to consider how intimacy is changing in digital contexts and what this might mean on a larger societal scale, in order to set the framework for the three different cases. Asking how intimacy is remediated, furthermore, points to the relevance of Jay David Bolter and Richard Grusin's concept of remediation (1999) for my project. It is a 
concept that I believe will prove useful for understanding those processes.

The second question shifts the focus to the three digital cultures that I have chosen to examine and their particular phenomena, and investigates their strategies for working against what I will describe as 'digital inimicalities'; that is, how the internet and its technocultures are sometimes rendered rather exclusionary or hostile domains, particularly for women and minorities.7 Here, the more political dimension of the project becomes visible. What I want to discover is thus whether digital intimacy approaches can actually become tools to counteract hate speech, exclusionary tech cultures, or objectifying visual cultures.

The last question, thus, seeks to determine how digital intimacy can be conceptualized for a feminist internet politics. This question is based on the assumption that a unified feminist positioning vis-à-vis particular digital inimicalities could be politically valuable. This dissertation aims to contribute to the theoretical development of such a positioning.

7 Of course this is a generalization: 'the internet', 'hostile' and 'for women' are all terms open to interpretation. For instance, some see the internet as another layer of the social while others think of material-technological aspects (or both). However, the question of where the internet starts and where it ends is as fashionable as it is open-ended. Also, the term 'women' as a monolithic unit has been taken apart in feminist theory, notably by Judith Butler (1990). Nevertheless, it is predominantly femininity and female-bodied or female-identifying persons and cultures that are affected in the contexts I describe here. I am therefore adopting a certain "strategic essentialism", a term borrowed from Gayatri Chakravorty Spivak, who used it in postcolonial theory (1990), which argues for a particular essentialism when talking about a particular group, that cannot be homogenized, for strategic reasons; for example, to make a political argument. Nevertheless, I aim for an intersectional approach, that is, to remain aware of how multiple sociocultural categories of inequality interfere, and how "historically specific kinds of power differentials and/or constraining normativities, based on discursively, institutionally and/or structurally constructed sociocultural categorizations such as gender, ethnicity, race, class, sexuality, age/generation, dis/ability, nationality, mother tongue and so on, interact, and in so doing produce different kinds of societal inequalities and unjust social relations" (Lykke 2010, $51)$. 
In this vein, I want to contribute to a growing and diversifying field of research. What I consider to be missing is a multi-perspective study of how women's and feminists' digital engagements try to counteract these inimicalities today and what analytical tools can be extracted from this. In a way, these discussions on different forms of digital inimicalities can also be understood as continuations of discussions begun by cyberfeminism. ${ }^{8}$

Or to put it into different words: In a special issue of Feminist Media Studies on Mobile Intimacies, Larissa Hjorth and Sun Sun Lim point out that "[w]hile the movement of intimacy towards the public was happening long before social and mobile media [...], the types of cartographies it takes within social, mobile and locative media requires explanation and elaboration" $(2012,478)$. I want to contribute to this cartography and also illustrate the political dimension of digital intimacy in the context of a digital feminist toolbox.

Next, I introduce the three different cases of digital cultures in more detail and position them in relation to the inimical backgrounds against which they are pitted.

\footnotetext{
${ }^{8}$ As a movement born at the beginning of the 1990 and at the intersections of feminist theory, media art, and online networking, cyberfeminisms were investigating, criticizing and intervening in gendered power structures, relations in digital media and other arenas of digital technologies, in deliberately creative, playful and ironic ways (Paasonen 2011). But cyberfeminism as an umbrella term for different kinds of academic and activist bodies of thought has lost its importance, or often is subsumed into feminist studies of digital media (ibid.). Even though one of the more consistent commonalities between different cyberfeminist thinkers and doers is a devotion to multiplicity and a refusal to define what cyberfeminism is in the first place, a set of critiques or paradigm changes might have contributed to the demise of cyberfeminisms from our screens, at least in this particular incarnation. So are the prefix 'cyber' and its related imaginaries more wedded to 1990 os discourses and seldom related to today's web 2.0 and its immense increase in user-generated content and mobile devices (ibid.). A lack of intersectional sensibilities, which often assumed an "educated, white, upper-middle-class, Englishspeaking, culturally sophisticated readership," and reproduced the "damaging universalism of 'old-style-feminism" was another common critique (Fernandez and Wilding 2002, 21). Alison Adam has called this The Ethical Dimension of Cyberfeminism (2002) and argues that cyberfeminism did not tackle ethical questions sufficiently and that it needed to become more politically engaged.
} 


\subsection{Hate and Hostility Online}

Early academic work on gender issues in CMC (computer-mediated communication), for example the linguistic work of Susan Herring (1996a; 1996b), has analyzed ways in which women online are more often addressed by harsh language and rough communication tones than men. Virtual ethnographic fieldwork in male-dominated online forums, on the other hand, has explored exclusionary tendencies and hierarchies in such communities (cf. Kendall 2002).

Academic work of this kind meant a break with some early discussions that took place during the 1990s, in which it was frequently argued that 'cyberspace' might be a place for disembodiment that lacks any place for sex differences (Wajcman 2010) or other markers of differentiation. The hope was that it might therefore be a liberating and equalizing place in which identity categories could be chosen and put on or off as one pleased ("identity tourism", as Lisa Nakamura phrased it, 2002).

The studies mentioned above pointed out that the internet is not a liberating playground for everyone, an insight that is well established today (cf. Adam 2002; Sundén 2003, 2007; Paasonen 2011b). As Stacy Gillis has summed it up:

The Internet does question the Enlightenment notion of self - as a gendered, raced and psychically sound individual [...]. But the cyber-body retains, for example, characteristics of gender and race because both are a social configuration. The body circulating through cyberspace does not obviate the body at the keyboard. The conditions for the cyber-dissolution of the body remain the gendered and racial body, so although the Internet raises questions about the Enlightenment notion of self by silencing once again the very question of embodiment, it also reifies the paradigms that endorse this selfhood. (Gillis 2004, 190)

The illusion of disembodiment online, just like the notion of 'cyberspace' itself as a particular space outside of everyday life or maybe even 
a "'room of one's own' that offers an escape from the constraints of being a gendered woman" (Daniels 2012, 30), was thus refuted in the early 200os. Since then, I would argue, the faces of online harassment and hostile digital cultures have changed and intensified in the context of the web 2.0 and the rise of social media in many, mainly more economically developed, parts of the world.

Monica Lewinsky has dated the beginning of this change to what happened to her roughly twenty years ago, by calling herself the "patient zero" (2015) of cyberbullying:

There was no Facebook, Twitter or Instagram back then. [...] But there were gossip, news and entertainment websites replete with comment sections and emails which could be forwarded. Of course, it was all done on the excruciatingly slow dial up. Yet around the world this story went. A viral phenomenon that, you could argue, was the first moment of truly 'social media'. (quoted in: Merica 2014, n.p.)

It is indeed interesting to think about how the affair of Lewinsky with the then-president of the USA was discussed and dissected in a public forum of, until then, unimaginable media outreach. It is almost impossible to comprehend what this public crucifixion and slut-shaming must have done to a 22-year-old woman who had "fallen in love with the wrong person" and who will deeply regret that mistake every day for the rest of her life, as she says (Lewinsky 2015). She tried to describe it: "I felt like every layer of my skin and my identity were ripped off of me in '98 and '99 [...] It's a skinning of sorts. You feel incredibly raw and frightened. But I also feel like the shame sticks to you like tar" (quoted in: Ronson 2016, n.p.).

Due to the rapid development of digital and social media, things have intensified in a context where digital media are more wireless, more mobile, and where it is becoming increasingly difficult to be offline. Lewinsky, who today works as an anti-cyberbullying activist, observes that "the landscape has sadly become much more populated 
with instances like mine, whether or not someone actually made a mistake, and now it's for both public and private people" (2015, n.p.). General awareness of the extent of the problem, which disproportionally affects women, racial others, and members of LGBTQI communities (Lewinsky 2015; Gardiner et al. 2016), has increased and the issue is discussed more frequently in both academia and public discourse (cf. Carstensen 2013; Marcotte 2014; Bücker 2014; Friedman 2014; Ganz 2015; Roth 2014; Hess 2014; Gardiner et al. 2016). At least two selfhelp books about how to handle hate speech online have been released, one in a US-American and one in a Swedish context (Blue 2015a; Bohlin 2016).9

The point is that online harassment and threats have become widespread. The social relevance of this problem lies in the danger that these hostile climates might silence female and marginal voices and force them away from participation, or at least make online communications less bearable for them. In any case, it makes living and working online more exhausting, less profitable and more time-consuming for affected people (Hess 2014). It is a situation in which it is common to see feminist activists harassed and their work discredited. As feminist journalist and writer Amanda Hess reports:

Threats of rape, death, and stalking can overpower our emotional bandwidth, take up our time, and cost us money through legal fees, online protection services, and missed wages. I've spent countless hours over the past four years logging the online activity of one particularly committed cyberstalker, just in case. And as the Internet becomes increasingly central to the human experience, the ability of women to live and work freely online will be shaped, and

\footnotetext{
9 Blue's book The Smart Girl's Guide to Privacy: Practical Tips for Staying Safe Online focuses, as the name states, on how to protect one's privacy, by explaining how to delete personal content from websites, set up safe online profiles, and what laws can protect users and how. In the book Tackla hatet, Swedish for Tackle the hate, Bohlin interviewed several journalists, public figures and researchers on how they have been victims of online hate and threats, and how they have handled it. The book goes on to analyze and give advice on how to deal with hate and threats, both online and offline.
} 
too often limited, by the technology companies that host these threats, the constellation of local and federal law enforcement officers who investigate them, and the popular commentators who dismiss them - all arenas that remain dominated by men, many of whom have little personal understanding of what women face online every day. (Hess 2014)

Despite a growing awareness of the problem, ${ }^{10}$ there still have been only a few studies or surveys capturing numbers and experiences of affected people and perpetrators. Within the framework of its ongoing web news reportage series "the web we want", the British newspaper The Guardian ${ }^{11}$ conducted its own research into online harassment and, by analyzing its database of the comments the newspaper has received online, provided

the first quantitative evidence for what female journalists have long suspected: that articles written by women attract more abuse and dismissive trolling than those written by men, regardless of what the article is about. Although the majority of our regular opinion writers are white men, we found that those who experienced the highest levels of abuse and dismissive trolling were not. The 10 regular writers who got the most abuse were eight women (four white and four non-white) and two black men. Two of the women and one of the men were gay. And of the eight women in the 'top 10', one was Muslim and one Jewish. (Gardiner et al. 2016) ${ }^{12}$

\footnotetext{
${ }^{10}$ Hess' article in the Pacific Standard has been considered by many to be an 'eye opener' about the severity and complexity of the issue.

${ }^{11}$ https://www.theguardian.com/technology/series/the-web-we-want, last accessed 01/07/2016. Another quantitative study is the PEW research center report (Duggan 2014).

${ }^{12}$ For an insightful report on their methodology, see: https://www.theguardian.com/technology/2016/apr/12/the-dark-side-of-guardian-comments, last accessed 27/04/2016.
} 
The point is that, even five years ago, online harassment and abuse were not treated as a major issue. Today, instances are more openly talked about and discussed, both in the media and at an academic level, and there is more support for anti-harassment initiatives and the tightening of laws. The different kinds of online harassment and hate speech aim at intimate issues, they aim to intimidate the addressee, to put their most intimate concerns on public display, and the understanding that this is an issue to be taken seriously is growing.

\section{Hatr: Countering Online Hate}

The problem of online hate and harassment is complex and multi-layered, and there are no quick fixes. As mentioned above, the options seem to be oscillating between self-help literature (which is undoubtedly empowering to a certain extent, but at the same time it passes the responsibility into the harassed person's own hands), and legal measures or policies adopted by the social networking service (SNS) operators.

The latter are not always effective and are often only implemented slowly and hesitantly, since many SNS struggle to find a balanced path between censorship and freedom of speech that does not repel users. I would thus like to suggest that what is missing is a critical and theoretical reflection from a feminist perspective that could build the theoretical foundations for a collective feminist call to action.

Interestingly, hatr is proposing an alternative to legal action or leaving the responsibility in the affected person's own hands. hatr is a small, little-known internet initiative that can be understood as an experimental answer to the question of how to handle online harassment differently. It is a platform that collects hateful comments posted by trolls and hate speakers in the comment sections of socially critical blogs, mainly feminist, anti-sexist and/or anti-racist ones, which are participating in the hatr project. These hateful comments are then displaced from their initial location and re-posted out of context on hatr.org. The strategy of collecting the comments in one central place 
makes them visible and creates a digital testimony. The idea is that the comments remain visible online, but do not disrupt the discussion climate of the particular blog and that - in an ironic twist - the hateful comments can be monetized for a good cause.

In my first empirical chapter (chapter four), I investigate this alternative approach and evaluate it as a potential tool for a feminist internet politics. I draw on Sara Ahmed's approach in The Cultural Politics of Emotion (2004), in which she investigates the relationship between bodies, language, and culture to illustrate how hatr.org uses strategies of affective politics and disrupts affective economies.

\subsection{Women in Tech 2.0}

Another approach to digital intimacy and digital inimicalities is to ask about the design processes of social media or social networking sites, and whether some ethical issues are related to how they are built. Amanda Hess, for example, makes a connection between online harassment and abuse and the ways in which online worlds are constructed, and to how a vast majority of the founders of internet companies, computer programmers, and software developers are not sufficiently considering ethical factors during the design process. "Most executives aren't intentionally boxing women out. But the decisions these men make have serious implications for billions of people. The gender imbalance in their companies compromises their ability to understand the lives of half their users" (Hess 2014, n.p.). In other words, the claim is that diversified perspectives could help to raise awareness of potential weaknesses or ethical hindrances that work to the detriment of particular groups.

Facebook, for example, has been called the least safe place for women and LGBTQI persons in a report by the US-American National Network to End Domestic Violence (2014). ${ }^{13}$ The social network's 'real

\footnotetext{
${ }_{13}$ "It is unsurprising that nearly every program reported Facebook as the main social media abusers use to harass victims. [...] Facebook is the hardest for survivors to shut down or avoid because they use it to keep in contact
} 
name policy', which Facebook has been following more and more rigidly since approximately 2012, supposedly to enable "a safer community for everyone" (quoted in: Blue 2015b), was implemented on the assumption that violations would become fewer if user accounts were connected to what Facebook called 'authentic names'.

This means that users with 'non-authentic names' can be reported, locked out of their accounts, have their profile names changed without consent or have to provide forms of identity legitimation - a practice that affects people who use different names than on their birth certificates, for example, gender-fluid people, survivors of stalking or harassment, or simply people who do not want to come out to their friends or family by displaying their social connections. As a consequence of the 'real name policy', some users have lost their profiles with all of their connections or have been forced to use names they are not comfortable with.

Other issues discussed in recent years have been the handling of hate and violence-promoting groups within social networks (cf. Gross 2013), or the fact that haters on Twitter can be blocked, but are able to create new accounts within minutes and can thus bypass the blockage (cf. Tung 2016).

The idea is often that more diversified groups of software developers and creators might offer different solutions or other insights when discussing these questions of social media ethics. The assumption is that a relatively homogenous group of developers has not taken the needs of women and racial others sufficiently into account because of unfamiliarity with the problems. Or, as social media theorist Nathan Jurgenson puts it, "Silicon Valley has the power to shape society to conform to its values, which prioritize openness and connectivity [...]. But why are engineers in California getting to decide what constitutes harassment for people all around the world?” (quoted in: Hess 2014, n.p.).

with other friends and family. [...] Although we often hear suggestions that survivors shouldn't use social media, we don't agree that this is a solution." (quoted in: Blue 2015b, n.p.) 


\section{Women*-Centered Coding Initiatives}

One attempt to change this situation of a gender imbalance in tech industries is to promote girls, women ${ }^{* 14}$ and other marginalized groups through setting up tailor-made coding groups, networks, and workshops. For example, Heather Payne, founder of the coding network $\mathrm{La}$ dies Learning Code said in an interview that software created by a small proportion of the population, "typically white dudes in their twenties", could hardly reflect the needs of a diverse society. Therefore, she argues that

women and [...] racial minorities, and older people, and younger people, have different needs and different things that they need technology to do, and by all of us participating in building it, whether it is open source projects or personal projects [...], that's how we're gonna create technology to serve a much broader population. ${ }^{15}$

In recent years, a few volunteer-run or NGO-based organizations like hers have popped up, aiming to bring women* together in small groups and workshops to stimulate their interest in digital technologies and the IT industry and to change normative attitudes towards coding, hacking, or other aspects of 'the inside of the machine'.

Examples include: the US-based organization Girl Develop It, which exists "to provide affordable and judgment-free opportunities for women interested in learning web and software development", ${ }^{16}$ the Canadian non-profit organization Ladies Learning Code who are

${ }^{14}$ The asterisk here symbolizes that not all of the introduced initiatives offer their courses only to women. Many explain in their mission statements that, for example, the groups are open to biological men if there are free spots, or if he brings a girl or woman along, that the groups are open to trans or queer people, to elderly people, and so on, and seek to generally increase diversity. However, as will become clearer in the course of the thesis, most of these groups appeal mainly to women and girls and to normative concepts of femininity.

${ }^{15} \mathrm{https}$ // $\mathrm{www} . y o u t u b e . c o m / w a t c h ? \mathrm{v}=\mathrm{QX} 8$ fxtt1rss, last accessed 16/10/2016, 1:05 min.

${ }^{16}$ www.girldevelopit.com/, last accessed 17/2/2014. 
"working to empower everyone to feel comfortable learning beginnerfriendly technical skills in a social, collaborative way", ${ }^{17}$ the originally Finnish and now worldwide acting volunteer network of the Railsgirls, which aims to "give tools and a community for women to understand technology and to build their ideas", ${ }^{18}$ and the Swedish Tjejhack ('Girlhack'), "working to provide girls and women with tools and support for computer science and creative digital work". ${ }^{19}$

These programming courses for women* are public spaces designed to confer a more intimate handling of contemporary technologies. Analyzing this emphasis on connectivity and intimate spheres might give an indication of what a 'doing digital media differently' approach could look like offline.

In my second empirical chapter, I frame the emergence of diverse coding initiatives and their cultural embedding as a societal trend. It is striking in this context how these initiatives discursively connect with intimate notions such as sociality, intimacy, and passion. I start out my analysis with exploring how the connection between intimacy and digital technology has changed historically through the example of women and coding. I analyze how notions of intimacy in relation to technology have changed - such as the idea of being intimate with the machine and what (digital) intimacy means, then and now. Part of this chapter draws on fieldwork conducted at a women*-centered programming course, as a way of framing the discussion of intimacy in and with the digital in a neoliberal context.

While the first two cases have a clear activist connotation (hatr was founded as a queerfeminist project countering hate speech online, and the women*-centered initiatives that I have introduced are interested in contesting the gender gap in digital technologies), my last case is rather different because it is centered around visual digital cultures and the role of the body in digital media.

\footnotetext{
17 http://ladieslearningcode.com/, last accessed 15/2/2014.

$18 \mathrm{http}: / /$ railsgirls.com/, last accessed 17/2/2014.

19 http://tjejhack.se/en/, last accessed 13/1/2016.
} 


\subsection{The Whispering Women of ASMR}

ASMR videos on YouTube are a rather quirky example of very explicit attempts to create intimacy and physical closeness online. ASMR stands for Autonomous Sensory Meridian Response and is a 'new' or 'newly discovered' human affect, that for some users can cause a "perceptual phenomenon characterized as a distinct, pleasurable tingling sensation in the head, scalp, back or peripheral regions of the body in response to visual, auditory, olfactory, and/or cognitive stimuli." ${ }^{20} \mathrm{On}$ one of the bigger community bulletin boards on reddit.com, ${ }^{21}$ affected people name and share stimuli that cause ASMR:

\section{COMMON TRIGGERS}

Slow speech patterns, accents, soft-speaking voices and whispers.

Lip sounds/smacking/eating

Clicking sounds, brushing sounds, white noise, etc.

Painting/drawing

Instructional videos

Watching other people performing simple tasks

Getting close, personal attention from someone (eyeexam, make-over, etc.)

Haircuts, people playing with your hair

Bob Ross

Hippie painter Bob Ross' instructional videos, which famously spawned a cult following, are often used as an example: The sound of his calming voice in combination with the brush strokes or scrapings with different tools on the canvas in his The Joy of Painting TV show can give 'braingasms' to some ASMRers. Since many of his videos are easily available online, ${ }^{22}$ many viewers discussed them online and

\footnotetext{
20 http://en.wikipedia.org/wiki/Autonomous_sensory_meridian_response, last accessed 30/10/2013.

${ }^{21} \mathrm{http}: / /$ www.reddit.com/r/asmr/, last accessed 25/11/2013.

${ }^{22}$ For example, you can find an 'ASMR playlist' of Bob Ross videos here:

http://www.youtube.com/watch?v=ugZgMIC-

nkew\&list=PL974B9758BoCF743D, last accessed 7/03/2014.
} 
thereby learned to define and express 'the feeling that did not have a name' (Stafford 2013) for the first time.

Anyhow, the phenomenon is not limited to brushes. Stars of the genre are usually conventionally beautiful young women, who offer the inclined user a multitude of ways to 'get the frizzles': Users can get their face cleansed by Jade ASMR Garden. ${ }^{23}$ They can watch and listen to The Waterwhispers scratching a carpet for more than 20 minutes. ${ }^{24}$ TheOneLilium can act as a loving friend who takes away users' worries and spoils them with a nice cup of tea. ${ }^{25}$ GentleWhispering offers a relaxing eye examination, which has been viewed more than a million times. ${ }^{26}$ One can cuddle and get ready for sleep with WhisperingRose $A S M R^{27}$, ASMR Massage Psychetruth whispers a goodnight story, ${ }^{28}$ and Heather Feather delivers a one-hour virtual haircut, a treatment that has been watched/experienced almost one million times. ${ }^{29}$

The practices/performances presented in these videos, made for (and often by) people who experience ASMR, are affective and build intimate entanglements of digital/non-digital worlds and embodiments. The performers, almost always self-identified women, give ASMR users 'tickles in the head' by carrying out certain tasks like whispering, lip smacking, brushing hair, or by giving more advanced roleplay performances which include a lot of performances of personal care. On YouTube, some of these performers, who are sometimes also called whispering women in news reportage (since whispering seems

$23 \mathrm{https}$ //www.youtube.com/watch?v=dSPW8iCycRE, last accessed 16/10/2016.

24 https://www.youtube.com/watch?v=HEmOwJid8NE, last accessed 16/10/2016.

25 https://www.youtube.com/watch?v=xBkGaMA9jTo, last accessed 16/10/2016.

26 https://www.youtube.com/watch?v=JcPoXFVV44c, last accessed 16/10/2016.

27 https://www.youtube.com/watch?v=_yyLTisC-so, last accessed 16/10/2016.

28 https://www.youtube.com/watch?v=7igsTFnZopo, last accessed 16/10/2016.

29 https://www.youtube.com/watch?v=4FW_s6VDWc, last accessed 16/10/2016. 
to be an essential part of the experience for many users), are downright starlets of digital intimacy.

\section{On Whispering and Gazing}

In my final empirical chapter (chapter six), I analyze the ASMR subculture as it is presented through and potentially amplified by digital media, particularly the video-distributing platform YouTube. The digital inimicality that builds the backdrop for this chapter is a classical feminist question of representation in the public sphere. That is, it reverberates second-wave discussions about the way in which women perceive themselves and are perceived by society, and how these views are created and perpetuated within dominant ideologies of gender difference and feminine and masculine ideals, and the role that, for example, the media plays therein (Pilcher and Whelehan 2004, 135). These questions here are transferred into the context of web 2.0 digital and social media and visual cultures.

The point is that much of social media is increasingly based on image production such as photos, videos, or avatars, and therefore "corporate to a large degree" (Paasonen 2011b, 348), and subsequently fully involved in the "contemporary struggle to define the self as both embodied and mediated by the body" (Bolter and Grusin 1999, 240).

In order to approach the ASMR subcultures and answer the questions above, I provide thick descriptions (Geertz 1973) of some ASMR videos as everyday internet culture and discuss them in relation to "haptic visuality" (Marks 2002) and their potential to refashion the male gaze (Mulvey 1989). I also discuss what such haptic visuality might mean in a broader societal setting when intimacy becomes digital intimacy in this way. 


\subsection{Digital Inimicalities and Doing Digital Media Differently}

In this dissertation, I take snapshots of three different digital spheres and draw connections between them. The digital cultures that I have selected are all engaged with changing understandings of intimacy. All of them are digital spaces where questions of femininity and women's participation in changing digital spheres are negotiated and re-negotiated. At the same time, they each represent initiatives or phenomena that seek to change the status quo, be it online harassment, the gender gap in computing, or questions of representation and haptic modalities in visual cultures. But how can they be seen as a more unified case against something?

In a recent publication on sexism, Sara Ahmed wrote in the editorial that sexism indeed is "a problem with a name",30 but that even though it "seems like some tangible thing, knowable in and from its constancy, something we come up against, repeatedly, it is remarkably difficult to pin down" (Ahmed 2015, 5). It is important to make it tangible, in order not to forget that it exists: "To give a problem a name can change not only how we register an event but whether we register an event" (2015, 8, author's emphasis). Furthermore, naming a problem makes it an explicit object of academic enquiry, which aims to generate new knowledge and understanding $(2015,5)$.

In the same way, the intention of this thesis is to make the different outgrowths of hostile climates in the digital sphere tangible, as well as to evaluate possible resistance strategies for feminist internet politics and actions. But even though sexism is part of the problems described, I do not believe that it would be sufficient to think only in terms of sexism when it comes to these symptoms of hostile online cultures. Even though sexism is one of the most toxic weapons used in such things as online harassment, I do not see digital inimicalities

3o Referring to Betty Friedan's descriptions of the "problem without a name" (Friedan [1963] 2001). 
solely as sexism. It is rather a complex meshwork of interlocking oppressions. In order to name the problems and their interconnection as an explicit object of academic enquiry and to generate new knowledge, I talk of digital inimicality.

To be inimical means to have "the disposition or temper of an enemy; unfriendly, unfavorable", to be opposed "in tendency, influence, or effects; antagonistic; inconsistent; incompatible; adverse; repugnant." ${ }_{11}$ Furthermore, according to dictionaries, inimicality, in contrast to hostility, is more frequently used in the context of private enmity (while hostility is more often used in the context of the public).

As an antonym of amicable, I think that inimical is a useful term because it allows relating to several inimical facets of digital cultures: objectification, exclusion, hostility, hate speech, and other oppressions at the intersection of gender, race, class, citizenship, sexuality, and other identity markers. In this way, inimicality is also about hierarchies: being inimical towards something means wishing to degrade the other and seeing oneself as incompatible with the other. In this way, inimicality acts as a counterpart to intimacy, and inimicality is often aimed at that which is regarded as 'different' from a normative standpoint. But what does 'differently' mean in the context of digital initiatives that do things 'differently'?

Thinking about difference, as feminist philosopher Rosi Braidotti has shown, has been a cornerstone of European history and philosophy, whilst "being different from" usually marks asymmetrical power relations. Someone or something is different to the "built-in standard of reference that constitutes the assumed norm" (2002, 158). For Braidotti, the aim of feminist practice is thus "to overthrow the negative, oppressive connotations that are built into the notion of difference and the dialectics of Self and Other" (Braidotti 2002, 159). In this vein, she embraces the fact that, in the context of postmodern thought, the 'other' has returned: "woman, the sexual other of man; the ethnic or

${ }^{31}$ From the GNU version of the Collaborative International Dictionary of English, https://www.wordnik.com/words/inimical, last accessed 03/05/2016. 
native other of the Eurocentric subject; and the natural or earth other of technoculture emerge as counter-subjectivities" (Braidotti 2002, 166).

In this way, doing digital media differently also seeks counter-subjectivities and new forms of difference that disentangle difference from the normative hierarchy, from a plain good/bad dualism, so that difference becomes another kind of difference. I see the approach of doing digital media differently as a response to what Braidotti calls "negative differentiation" in the following quote:

It is therefore crucial to expose the logic of economic exploitation that equates nomadic flux with the profitminded circulation of commodities. Given that technologies are so intrinsic to the social and discursive structures of post-industrial societies, they deserve special attention. Access to and participation in the new high-tech world is unevenly distributed worldwide, with gender, age and ethnicity acting as major axes of negative differentiation. (Braidotti 2002, 168)

Digital media as part of such a high-tech, post-industrial world thus also becomes an arena for negative differentiation, not only through access to hardware (the "digital divide", cf. Norris 2001) but also through negative differentiation along the lines of harassment and exclusion, which limit access to contextual participation. In other words, my three cases - in very different, sometimes tentative, experimental, or only theoretical ways - try to work against forms of negative differentiation.

\section{Outline of the Book}

Following this introduction, chapter two is my theoretical chapter. Here, I approach my main theoretical concepts, analytical tools and lay out my conceptualization of digital intimacy. After this, in chapter 
three, I lay out my methodological approaches: I situate the thesis epistemologically, describe the points of departure for approaching my three strategic examples, and explain my overarching methodological framework and the methods that will be applied in each empirical chapter.

Chapter four is my first case study, on online hate speech and harassment. Here, I first locate the problem in a context of feminist digital activisms and their responses to such problems more generally, and then move on to hatr.org as a specific contemporary example. I analyze hatr's strategies in the framework of affective economies (Ahmed 2004) to finally discuss those strategies as potential tools for a feminist internet politics.

Chapter five is my second empirical chapter, which firstly locates women*-centered coding initiatives as broader societal trend. I then briefly trace the history of women and coding by discussing selected historical examples from the relationship of women and coding, analyze the use of intimate notions and their promises in women*-centered initiatives' promotional material, and finally move on to describe and discuss my own participation in the workshop of such an initiative.

My last empirical chapter, chapter six, features thick descriptions (Geertz 1973) of selected ASMR video clips, contextualizes them with feminist film theory and its successors, and ends with a discussion of ASMR's reverberations in a broader societal context of neoliberalism.

Chapter seven is the conclusion, in which I tie together the findings of all previous chapters and point out possible directions for future research. 


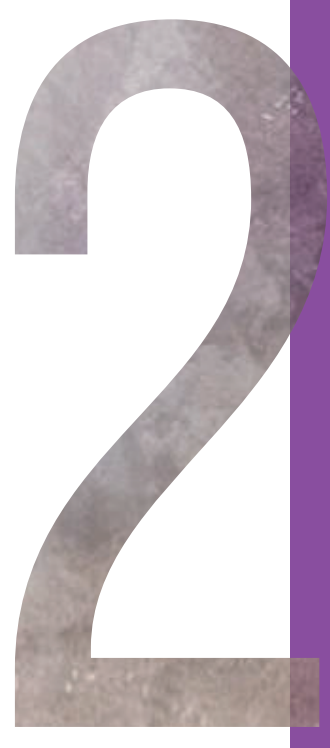

THEORIES OF INTIMACY 



\section{Theories of Intimacy}

In this chapter, I map out the theories that inform this thesis. I begin with the ways in which intimacy can be approached from a general perspective, tracing it from traditional to more recent understandings. I then turn to more theoretical discussions of intimacy, and lay out how intimacy has been discussed academically and the roles that it plays in mediated contexts. I also elaborate on its relation to digitality and explain how I see affect theory in relation to intimacy in general, and my research endeavor in particular. Finally, I define my own theoretical take on 'digital intimacy'.

\section{What's in a Kiss?}

'Intimacy' is a concept that is hard to pin down. This is why I start my exploration of what digital intimacy might entail, or what it might become, by describing a small, mundane gesture that most people would call an intimate one: a kiss. A kiss is an embodied contact and, at the same time, a culturally constructed, semiotically readable act. My key example of this is the socialist fraternal kiss between Erich Honecker (German Democratic Republic) and Leonid Brezhnev (Soviet Union), which happened in October 1979. It was an exchange of microscopically small drops of salvia and skin cells; a sign of affection and passion; as here, when performed between white heterosexual men, a symbolic action used in the Russian Orthodox Church. Furthermore, it was a physical contact uniting bodies; a stimulation of uncountable nerve cells; a symbolic act to signify intimacy between two countries, and thus a performative act (cf. Austin 1975) intended to create and sustain abstract concepts such as fraternity, equality, solidarity, and peace between the countries.

Some argue that the socialist fraternal kiss is not worth investigating or discussing, a ridiculous topic, or that it basically does not exist, 
that it is not a 'thing'. This is what Claudia Schimmel was informed when she wanted to write a political-historical paper on the subject, and asked a spokesman of the present-day Party of Democratic Socialism and the former chief of protocol of the German Democratic Republic at the end of the $1990 \mathrm{~s}(1998,81)$. The socialist fraternal kiss becomes peculiar because it does not follow the logic of a homosexual act, in the sense that this kiss between two men is not supposed to be read as such. It is an intimate gesture and one that is staged and performed at the same time, just like putting a signature on a document.

Additionally, this kiss became a historic symbol, captured by Régis Bossu's camera; it was re-published in the news of the time in various media, and subsequently in innumerable history books. Later, it was remixed into art, and painted onto the Berlin Wall, where it became a symbol of the downfall of the Soviet Union; thus, its initial message of a strong, political union was turned upside down.

Today, the painting has become a tourist attraction, and is often read in the vein of pop art or street art. Painted originally onto the Berlin Wall in spring 1990, the artwork was removed in 2009 because of renovation work on the wall, and then re-painted by the artist himself (Dmitri Wladimirowitsch Wrubel). On 29th April 2014, a photograph was taken of the artist cleaning his artwork in front of spectators..$^{32} \mathrm{On}$ this picture, the words "Homophobia in Russia" painted onto Brezhnev's right temple (presumably by an unknown street artist) are visible - once again, the intended message of this kiss has been reversed, remixed, and politicized, this time explicitly relating to homosexuality. Today, both the photograph and the painting are frequently re-printed onto countless coffee mugs, T-shirts, and other memorabilia of what in today's Germany is simply called 'die Wende' ('the turn' that led to the downfall of the socialist regime and paved the way for parliamentary democracy and ultimately led to the fall of the Wall).

$32 \mathrm{http://www.scmp.com/photos/recent/all/1499355,} \mathrm{last} \mathrm{accessed}$ 25/01/2016. 
The point for me is that the socialist fraternal kiss between Honecker and Brezhnev remains an intimate gesture, even though it happened in public, even though it had manifold different meanings besides the connotation of a lovers' gesture, and even though it has been replicated and commodified right up to the present day as a symbol of times gone by. All of these artifacts and reprints still reverberate long after the two pairs of lips touched. Following this, I would like to suggest that this kiss can be seen as a 'both/and' occurrence, an act that is more than the sum of its parts, that it is different from only being a kiss in a traditional, romantic sense.

\subsection{Intimacy}

In a similar way, intimacy, intimate issues, and violations of intimacy in a context of digitalization are open to constant transformation. Just as the fraternal kiss is a multitude of things, ranging from drops of salvia to a performative political act to a digital thumbnail image in a browser search, intimacy in the context of digitality has many different faces, such as intimate relationships with digital devices, the display of intimate affairs and affronts in online public forums, or the intimate visual affectivity of videos, gifs, and memes.

Intimate moments are often volatile, subjective, and unique and therefore the term can have manifold and at times even contradictory meanings. 'Intimacy' can describe a multitude of fuzzy feelings, wellplanned or unexpected encounters, and relationships with others or with things. It is personally experienced but frequently also a topic of public interest. Often, intimacy is understood in relation to sexuality and kinship - 'intimates' is a word that could describe your best friends or your underwear. In any case, it is often something that many people do not want to share with everybody.

Intimacy is culturally specific; its display can be considered provocation, or grounds for vulnerability, shame, political arguments, or protest. Intimacy has often been used to establish distinctions between public/private or local/global, where "the intimate stands apart from 
the state and the market, often contrasting the authenticity of local life with external impositions; that is, modern, Western, or capitalist forces invade intimate life" (Wilson 2016, 249).

In an intimate relationship, you know more about the other than an 'outsider' does; you know the other through the body or maybe even by heart. An intimate situation always depends on this other; even when being intimate with oneself, the situation depends on an imagined other of whom one is aware and whose (non-)presence has an impact on the situation ("What would they think if they were here?"). Solitary intimacy depends on what is considered, or what one considers to be, 'presentable'. It is thus defined in the absence of the other and depending on this absent other.

The Cambridge Dictionary of Sociology (2006) states that intimacy is a relatively new term in sociological theory; earlier, as author Carol Smart explains (2006, 308), it was often subsumed into discussions of private and public spheres, and in relation to family, romantic love, and marriage, usually from a heteronormative perspective (cf. Luhmann 1986). However, Smart draws attention to feminist research into these domains, which criticized the oppressive potential of institutionalized close contact relationships and brought discussions of queer relationships into the academic sphere, thereby triggering an increased interest in discussing intimacy from different and non-normative angles. Nevertheless, Smart stresses Anthony Giddens' The Transformation of Intimacy (1992) as being responsible for the eventual re-appropriation of the term.

Giddens also picks up on women's new demands for relationships and intimacy that have been uncovered by emancipation projects, such as second-wave feminism, and discusses examples like same-sex marriage. The changing roles of friendships, casual encounters in times of high divorce rates, declining childbirth rates, and the increasing number of single-person households might be other reasons for an awakened interest in intimacy as a concept.

And indeed, in feminist and queer scholarship, and in gender studies, intimacy has been a familiar analytical category, because questions of sexuality, social relations, and kinship were often their point of 
departure, their main topic, and/or inseparable from feminist epistemologies and methodologies. From the foundational declaration that 'the personal is the political' to feminist standpoint epistemologies (Harding 2004) and situated knowledges, to intimate forms of doing fieldwork (Dahl 2010), to the cyborg as a way to understand intimate engagements with technology (Haraway 1991) and to scholarship that invites to think through the skin (Ahmed and Stacey 2001): Feminist thinking and writing has intimacy written all over it. This is also a response to the fact that passions, emotions, or feelings historically have often been represented "as 'beneath' the faculties of thought and reason" (Ahmed 2004, 3). As a response, handling intimate issues in intimate ways has been turned into a strength in feminist theorizing.

Generally, the word intimacy describes close relations. It is usually used as a 'synonym of a concept' for things happening at the local, micro level, in private and on embodied levels, and on levels that involve the psyche in one way or another (Wilson 2016, 249). If something is too intimate, like a personal question, one wishes to keep it to oneself, within or close to one's own body, as something that is embodied. A personal opinion or condition that makes oneself attackable is intimate; stories of illness, close relationships, sexuality, or stories considered to be embarrassing are intimate.

In short, situations where one is 'not put together' but spilling out and leaking (Shildrick 1997) are intimate. Getting intimate with someone or something means crossing a boundary and connecting with the other, and being at risk of losing oneself to some degree. Intimacy is thus also about transgressing the boundaries of the embodied self and the boundaries of discursive norms. Intimacy can be experienced in the context of oceanic feelings like love or it can be a brief temporal attachment, like watching an erotic movie or caressing a neighbor's dog. Intimacy requires a particular knowledge about the other, a kind of access to the other. Intimacy depends on mutuality: it can never be a one-way street, even when the other might not be geographically close. 


\section{Public Intimacy}

Since intimacy is a term that can mean so many different things to different individuals and in diverse social, cultural, spatial, temporal, historical, academic, and other intersecting contexts, Lauren Berlant, professor of English at the University of Chicago and often the first port of call when intimacy is discussed, is sure that in the end "virtually no one knows how to do intimacy", even though "everyone feels expert about it" $(1998,282)$.

Her point is that, in spite of nobody knowing how to do intimacy, it is done everywhere, and it is done publicly: discussed in talk shows, self-help literature, and group therapy sessions. From her point of view, the only thing that seems to be certain about intimacy is that its oscillation between desire and therapy has become a mass-mediated event (ibid.). Berlant was writing at a time prior to the immense expansion of digital and social media that are the focus of this particular text, and it can be said that forms of performing intimacy publicly have multiplied and diversified immensely over the last two decades, due to processes of mediatization and digitalization.

The concept of a kiss has been transformed from a set of narrow categories - from an embodied gesture frozen in a particular temporal and spatial setting to, in the case of Honecker/Brezhnev's oral conjunction, a historically relevant and frequently replicated artwork. This latter is an artwork which, following Walter Benjamin, is at risk of losing its aura, its "here and now of the work of art - its unique existence at the place at which it is to be found" (Benjamin [1936] 2010, 13) through technological reproducibility. In a similar fashion, intimacy's mediation as described by Berlant has been extended, multiplied, calibrated, enhanced, and accelerated in an age of digital reproducibility. But does this mean that intimacy is losing its 'aura', its special, magical something? Where is the 'here and now' of intimacy when it is digitalized?

As a lowest common denominator in this quest to find a working notion of intimacy, I would like to suggest that, in the broadest sense of the term, intimacy describes a context that is relational, and that this 
relation affects one's body and embodied self. In this broad definition, intimacy thus becomes a close, affective relation. In her understanding of intimacy, Berlant tries to see it as something much broader than "within the purview of institutions, the state, and an ideal of publicness" (Berlant 1998, 284), and she asks:

What if we saw it emerge from much more mobile processes of attachment? While the fantasies associated with intimacy usually end up occupying the space of convention, in practice the drive toward it is a kind of wild thing that is not necessarily organized that way, or any way. It can be portable, unattached to a concrete space: a drive that creates spaces around it through practices. The kinds of connections that impact on people, and on which they depend for living (if not 'a life'), do not always respect the predictable forms: nations and citizens, churches and the faithful, workers at work, writers and readers, memorizers of songs, people who walk dogs or swim at the same time each day, fetishists and their objects, teachers and students, serial lovers, sports lovers, listeners to voices who explain things manageably (on the radio, at conferences, on television screens, on line, in therapy), fans and celebrities - I (or you) could go on. These spaces are produced relationally; people and/in institutions can return repeatedly to them and produce something, though frequently not history in its ordinary, memorable, or valorized sense, and not always 'something' of positive value. Intimacy seen in this spreading way does generate an aesthetic, an aesthetic of attachment, but no inevitable forms or feelings are attached to it. (Berlant 2011, 284-85)

What Berlant is doing here is extending the idea of intimacy. Detached from its institutionalized forms, she sees it as a much more mobile form of attachment, a 'wild thing' that might be unpredictable and unattached to concrete spaces. A mobile attachment - which sounds a bit like a contradiction - describes the aesthetics of attachment, rather than its definitive execution. Things 'come together' in a particular way 
and relate to each other. These attachments are much less predictable than the bourgeois construction of intimacy leads us to believe - a perception that ideologically obscures how political the historically and socially constructed separation between public and private is and how othered groups have been systematically excluded from it (Meßmer, Schmidbaur, and Villa 2014, 3). What becomes a constant value, on the other hand, is the aesthetic principle that intimacies are created relationally and that they produce something, 'valuable' or not. This also means, according to Berlant, that what is produced is not necessarily related to feelings or emotions. If intimacy is understood in this way, it allows us to see romantic or family relationships, sexuality, or institutionalized forms of intimacy as optional and mobile attachments, which move within a non-predefined matrix of bodies, people, objects, or technologies. In this way, intimacy becomes a rubric that allows for an "object pluralism that is able to attend to an array of entities (e.g., animals, things) in a range of relations (nonheterosexual, nonfamilial)" (Wilson 2016, 252). Therefore, as feminist scholar Ara Wilson argues, intimacy also becomes a 'placeholder':

The hope is that the colloquial term intimacy might avoid reproducing pitfalls now associated with concepts like sexuality or gender [...] - such as reifying female identity, relying on a heterosexuality/homosexuality binary, or assuming that true selves lie beyond power - while still allowing scholars to study recognizably erotic and gendered realms, including reflection about their categorization in the same analytical frame. The desire to resist forms of knowledge that perpetuate or rationalize global inequality (e.g., ideological reifications of family, sexuality, community) motivates the use of intimacy as a rubric. (Wilson 2012).

For the purpose of this dissertation, I am adapting this broadened understanding of intimacy, in the form of mobile attachments, following Berlant. This means that the intimate does not always come in predict- 
able forms and does not always create something 'of value'; it also becomes a 'placeholder', as Wilson has it - as an approach to avoid binaries such as public/private, hetero-/homosexuality, and so on. It becomes a flexible reference that describes relational lives and spaces beyond public/private distinctions and institutionalized forms of intimacy dictated by states and markets. However, thinking intimacy as a "provisional reference that emphasizes linkages across what are understood to be distinct realms (scales or entities)" (Wilson 2012) invites a conversation with affect theory, to which I will turn in the next section.

\section{Affect and Intimacy}

Similar to Berlant's list of uncontrollable situations in which intimacy appears, film scholar Marco Abel has described how responding to the outside world can never be a response without affect:

we never read a novel, poem, or essay, view a film or an advertising billboard, listen to a song or a cat's purr, sense a pinprick on our skin or the deep bass waves emitted by a high-end subwoofer in the trunk of a car passing by, or engage another person outside of the realm of affect. (Abel 2008, n.p.)

So what is the understanding of affect here, and how does it relate to intimacy? The affective turn in cultural studies, which has been going on roughly since the mid-9os, is concerned with the subject's discontinuity with itself. Affect is most often understood as a pre-personal, non-subjective, autonomous potentiality, intensity, or force. In other words, an affect could be a "gut reaction" or another sensation that does not fit into any fixed definition of emotion (Hillis, Paasonen, and Petit 2015, 1).33

33 There have been different directions taken by the affective turn: Clough names approaches that focus "on the circuit of affect and emotion" (Clough 2010, 207) and understand affect and emotions as opposite ends of a spectrum (Ahmed 2004; Sedgwick and Frank 2003). In the psychological school, 
Much of the theory on affect is grounded in Baruch Spinoza's use of the term (affectus). In his anti-Cartesian philosophy, mind and body are the same and they are both saturated with the sense of a direct bodily awareness. In Ethics ([1677] 1996), Spinoza was concerned with how bodies are influenced, molded, and changed during encounters with other bodies and how the experienced intensities influence one's lifeforces in different ways. Spinozian bodies, and thus minds, as Genevieve Lloyd concludes, make "our awareness of our bodies something very different from a detached, factual understanding of clearly circumscribed material entities. It becomes, rather, a confused awareness of other bodies grasped together with our own" (Lloyd 1996, 53). And it is this grasping together of other bodies with our own that offers a more intimate conception of how we come to know the world.

While Spinoza's affect was relatively similar (but not equivalent) to feelings and emotions, more recently the concept has been elaborated by thinkers such as Henri Bergson, Gilles Deleuze, Félix Guattari, Rosi Braidotti, Elizabeth Grosz, and Brian Massumi, in what is sometimes called the new materialist school of thinking, which is unified by an emphasis on bodily matter and matter generally. Deleuze developed an account of affect that, to a greater extent than Spinoza, disentangles emotion and affect.

This is part of his larger project, which aims to "understand, and comprehend, and express all of the incredible, wondrous, tragic, painful, and destructive configurations of things and bodies as temporally mediated, continuous events" (Coleman 2010, 11). A prerequisite for understanding bodies as this kind of temporally mediated, continuous events are two important and simultaneous qualities of the body, as defined by Deleuze through his readings of Spinoza.

The first is that a body, "however small it may be, is composed of an infinite number of particles; it is the relations of motion and rest, of

for example for Silvan Tomkins, the affective system is a biological system of input and output and the word affect describes emotional-corporeal and psychological reactions and states of being, such as euphoria. 
speeds and slowness between particles that define a body, the individuality of the body" (Deleuze 1988, 123). This kinetic proposition defines the body as a "complex relation between differential velocities, between deceleration and acceleration of particles" (ibid.). The second proposition is that "a body affects other bodies, or is affected by other bodies; it is this capacity for affecting and being affected that also defines a body in its individuality" (ibid.). To him, both capacities - the relation between speed and slowness, as well as the capacity to affect or to be affected - change the understanding of bodies from substances or subjects to modes.

This shift in perspective enables to "define an animal, or a human being, not by its form, its organs, and its functions, and not as a subject either [...] but by the affects of which it is capable" (Deleuze 1988, 12324). Consequently, in Deleuze's thinking, bodies that are affected and affecting are not solely human bodies. A body can be "an animal, a body of sounds, a mind or an idea; it can be a linguistic corpus, a social body, a collectivity" (Deleuze 1988, 127).

Coming back to the question of intimacy, it is becoming clear that intimacy is always affective. A collision of bodies (which might also be bodies of thought, technological objects, or collectivities, as Deleuze explained), is an intimate encounter in which bodies are modified through their encounter with the other.

Berlant's intimate attachments are mobile, and thus rely on bodies' velocities and bringing bodies into the moment of transition from one state to another or into the interval between transformations. Nevertheless, intimacy is not only an affect. Intimacy can be seen as what Deleuze described as affection: "the additive processes, forces, powers, and expressions of change - the mix of affects that produce the modification or change in the affected body" (Coleman 2010, 11). Affects are thus a prerequisite for a situation to become intimate. Affective intimacy can furthermore be seen as an intimacy that is not necessarily related to feelings and emotions (although they might emerge in the mix of affects, which is affection).

In the following, I will think intimacy through affect and as affective, and will use the terms attachment and affect interchangeably. 
From my point of view, both describe "capacities beyond the body's organic-physiological constraints" (Goldberg 2012, 242) and emphasize the entanglements of bodies with the world.

In this section, I have explained my take on intimacy and how I understand its relation to affect. In the following one, I describe my take on how intimate attachments are transferred, or remediated, into the sphere of the digital.

\section{Remediating Intimacy}

A crucial aspect of the spreading of intimacy into the online sphere is the remediation capacities of digital media: they transform and multiply the ways in which intimacies are narrated and performed, be it through websites and networks or other forms of sharing intimacies, such as (video-) telephony or SMS services. But what does remediation mean in this context and how can intimacy be remediated?

In the following, I am drawing on an example from a few years ago in which the expression 'digital intimacy' was employed in a newspaper article in the New York Times (Thomson 2008), in order to illustrate what a remediation of intimacy might look like. The author used digital intimacy as an umbrella term to describe particular phenomena via digital communication platforms that construct a kind of 'co-presence':

[L]overs who were working in different cities would send text messages back and forth all night - tiny updates like "enjoying a glass of wine now" or "watching TV while lying on the couch." They were doing it partly because talking for hours on mobile phones isn't very comfortable (or affordable). But they also discovered that the little PingPonging messages felt even more intimate than a phone call. (Thomson 2008, n.p.)

How can text messages, only consisting of a few words, be more intimate than a phone call? The article quotes information scientist Marc Davis, who proposed that the intimacy in this kind of communication 
lies in the fact that no message is the single most important message. Instead, the often rather random and en passant short messages are compared to just sitting next to somebody while doing your own thing, maybe smiling, "just [to] sort of let people know you're aware of them" (ibid.). Social scientists have named the phenomenon ambient awareness or ambient intimacy: "Just as physical proximity allows one's mood to be interpreted through a series of little behaviors (e.g., body language, sighs, stray comments), several tweets together can generate a strong feeling of closeness and intimacy" (Kaplan and Haenlein 2011, 107). The assumption is that ambient awareness helps users to feel less alone and to compensate for missing body language in these text-based communications and that the popularity of social network services such as Facebook or Twitter, which encourage the user to constantly update what they are doing, can be seen as a way to remediate intimacy. But what does remediation mean in this context?

Jay David Bolter and Richard Grusin's theorization of the term remediation (1999) contains two opposing but interrelated features: The first is immediacy or transparent immediacy and refers to the user's desire for the medium to disappear or to become imperceptible, in order to experience immediate, 'unfiltered' content. This desire is fulfilled when, for example, a good movie makes the viewer forget about her surroundings and draws her into the story and scenery, or, in the digital social media context described above, when ambient awareness occurs.

According to Bolter and Grusin, it can also be related to a desire for photorealism, in the sense of a demand for pictures to be as authentic as possible $(1999,28)$. Similarly, today it can often be witnessed in the race for the most convincing virtual reality technologies: conferring the illusion of transparent immediacy is often the goal in the design process (and also becomes, as I will explain in Chapter Four, a feature of ASMR videos).

The second aspect of remediation is hypermediacy. The term describes the fascination with media as a representational practice and cultural logic, as a visual style of representation that reminds the user of the medium. Bolter and Grusin mention, for example, the frames of 
the 'windows' that pop up on computer screens and thus remind users that the content being consumed has been 're-framed' in a digital window context (1999, 31).

Hypermediacy suggests a way to see developments in the media as not linear, but cyclical: media do not necessarily replace each other, but newer and older media, such as painting, photography, film, and television remix, co-exist with, and depend on each other. 34 Understanding media processes like this stresses the continuities of 'conventional' media. Framing hypermediated media as translating each other thus interrupts the utopian feeling of exploration that has surrounded cyberspace, early internet discourses, or technologically determinist views of computing in the past.

Coming back to the question of how intimacies are remediated, both aspects, hypermediacy and transparent immediacy, play a role in the negotiation of digital intimacy as it was discussed in the New York Times article. Intimacy is here hypermediated through the adaptation to a particular technology (in this case texting, in adjusting to a particular text format, a particular frequency of writing, embedding photos, videos, links, and so on). In this way, older media, for example, letters, notes, phone calls, and in this particular example maybe even body language or mundane spoken observations, are translated into another medium. While there is an initial adaptation process when one is getting used to the new tools and their specifics, transparent immediacy is often the desired goal for media makers and users; that is, in the examples above, the lovers who do not want to waive intimacy simply because they are not in the same geographical location. 35

34 One medium becomes translated into another one and/or one medium becomes the content of other media. One example could be the trend to stream movies or TV series online instead of turning on the TV and the reaction of broadcasting stations, which have been forced to expand their online presence and to offer their TV formats online as well.

35 In this context, it is interesting that André Jansson and Paul Adams argue that processes of mediatization and digitalization are transforming understandings of key geographical concepts such as space, place, territory, borders, movement, and mobility (2013). These transformations occur during the process of appropriating digital media and result in micro-changes in the patterns of social life; for example, when mobile media devices "permit one 
Since I am concerned with the question of how intimacy is remediated in the context of everyday online cultures, how the digital refigures intimacy, and what the coming together of both terms means, I am using Bolter and Grusin's iconic concept of remediation as a stepping stone, in order to clarify that I see intimacy as remediated into digital intimacy.

While Bolter and Grusin had a wide conception of what counts as media (for example, they also considered painting or the written word as media that refashion other media in cyclical movements), I am going a step further by postulating intimacy as a remediable asset and thereby, indirectly, as a medium itself, keeping in mind that intimacy is always an exchange of some sort that depends on the existence of an other. In this sense, I am agreeing with Amparo Lasén and Elena Casado (2012), who argue in their analysis of the remediation of heterosexual couple intimacy in the context of mobile telephony that

[d]igital mediations do not give rise to fully new exchanges, relations, activities and situations. Mediations produce forms of attachment: among bodies, among people, and between people and technologies. These mediations are operated by a full array of media, technologies, materialities, objects, as well as other immaterial mediations such as language, rituals or beliefs. Therefore, when digital technologies, such as mobile telephony, take part in the shaping of our intimacies, they remediate it. (Lasén and Casado 2012, 551)

One point that Lasén and Casado are making here is that digital mediations do not fully alter intimate exchanges. Rather, they are hypermediated in Grusin and Bolter's sense; that is, translated into another medium while refashioning older forms (one might think, for example, of

sensory mode to disengage from the surroundings through which one is moving and remain immersed in a stream of stimuli originating in another time and place"; when ideas of 'close' and 'far', of 'being together' begin to blur; or through the use of (self-) surveillance and (self-)tracking apps and devices, which mean "new forms of systemic power over the privacy, timing, and spacing of social practices" (2013, 305). 
phone calls or written letters in the context of couple intimacy). Furthermore, they suggest that mediations of this kind produce attachments: among bodies, people, technologies, and immaterial factors.

I see the concept of remediation as useful because it allows considering all mediation as remediation. In the context of intimacy, this means that intimacy is no less real when it is remediated, it does not become a fully new exchange, relation, activity, or situation, as Lasén and Casado assess in the quote above. Thus, intimacy is just hypermediated, it simply comes in a new guise but is not inherently new only because it is digital or happening in 'new' media.

My understanding of remediation also draws upon Sarah Kember and Joanna Zylinska's extension of the concept. In their book Life after digital media (2015), they propose thinking about remediation in a more open-ended fashion; that is, outside the framework of media (in the more traditional sense of media or digital media). They argue that remediation has the potential to be opened up for a non-anthropocentric approach. As an example, Kember and Zylinska point out the role hypermediacy played in the mediation of media events such as the flu pandemic, The Arab Spring, or the global credit crunch:

[A]ll of these events reveal a complex relationship between the event 'itself' and its mediation, foregrounding the significance of technological, social, economic, geographical, and other influences or forces well beyond those controlled by the human. This complexity demands an interdisciplinary nonhumanist theoretical framework that would facilitate such a multifaceted understanding. $(2015,9)$

They think that Bolter and Grusin's concept of remediation allows them to "open up the possibility of a nonhumanist reading of media as 
dynamic, complex and interwoven processes beyond the singular control of the human" $(2015,9)$, even though they later argue that Bolter and Grusin at times fail to make the most of this opportunity. ${ }^{36}$

I would like to argue that this take on remediation allows a connection with affect theory. As a consequence, remediatization can be understood in the sense of affection as a collision of bodies (which might also be bodies of thought, technological objects, or collectivities, as Deleuze explained) and also as an intimate encounter in which bodies are modified through their encounter with the other.

At the same time, the notion of transparent immediacy "as the goal of any medium or mediation mechanism" (Bolter and Grusin 1999, 25) is actually a tricky one in relation to an embodied account of remediation. The wish to arrive at the unmediated real (as in transparent immediacy), as Bolter and Grusin explain, may also "reflect the denial or subordination of the body in Western definitions of the self", which is thus an object of feminist critique $(1999,237)$. They relate this to Laura Mulvey's theorization of the male gaze as abstracted and disembodied (Mulvey 1989) and argue that

new media are thus fully involved in the contemporary struggle to define the self as both embodied and mediated by the body. On the one hand, they contribute new strategies of transparency that would seem to reinforce the dissecting male gaze. [...] On the other hand, through strategies of hypermediacy, digital media refashion the normative gaze and its implied views of male and female identity. (1999, 240)

I understand digital intimacy in this vein: as meandering between strategies of immediacy (as an urge to transgress the material and de-

${ }^{36}$ Ultimately, Kember and Zylinska stick with the term mediation instead of remediation because this "term highlights the ongoing aspect of the mediating process without circumscribing it too early either by human desire and action or by specific media" (Kember and Zylinska 2015, 19; 2015). However, for the purpose of this thesis I stick with remediation. 
fine the body as embodied and mediated at the same time) and the interrelated responsibilities that come with it, and strategies of hypermediacy that are reminiscent of the cultural logics resonating in the use of the technologies at hand. Those strategies of hypermediacy, Bolter and Grusin argue, can ultimately be used to subvert a normative gaze: "Because transparency always passes into hypermediacy, these same digital media can both enact and critique traditional beliefs about gender and self" $(1999,340)$. With the concept of digital intimacy, I thus want to demonstrate how both the enacting and the critique are acted out in my strategic examples. Where are those traditional gender beliefs perpetuated, and where are they scrutinized or deconstructed?

But first, I will give an account of my understanding of digitality in the following section.

\section{Intimacy and Being Digital}

In the previous section, I have elaborated upon the remediation capacities of digital media and how, as Couldry puts it, they enable transmissions, retransmissions, and transformations (2008, 374). Such capacities are deeply embedded in the logics of digitality itself. Then again, what this means may not be obvious. 'Digital' is yet another umbrella category, and it is used to describe various forms and modes that have evolved in relation to the advent and development of computer technologies. Today, the term is often used interchangeably with 'digital media', 37 because of the significant shifts and developments over the last few decades within the area of digital media. Examples of such shifts include media convergence, the mediation of identities, the redefinition of social boundaries, and the transcendence of geographical boundaries (Markham and Baym 2009, 57). However, as internet researcher Harry Halpin points out,

37 The term 'digital media' incorporates virtually any and all media in a machine-readable format. Examples of digital media range from computer software, visual, auditory, and interactive media such as video, music, or video games to websites, including social media. 
shockingly, there is no clear notion of what 'being' digital consists of, even though a working notion of digitality is necessary to understand computers, if not human intelligence. This is not to say that 'digitality' is not understood in a practical or engineering sense, for assuredly we build digital systems. While engineers can implement digitality, and ordinary people 'know it when they see it,' there is no rigorous philosophical definition of digitality. $(2013,199)$

This uncertainty in the understanding of what 'being digital' actually consists of sometimes leads to a certain 'digital mysticism', which is "a special brand of technological determinism in which digitality and software are considered to be ontologically immaterial determinants of new media" (van den Boomen et al. 2009, 10). This is not the case. Halpin explains that far from being immaterial,

digitality can be defined as relationship from an encoding to content where the encoding is finitely differentiable and the type of the encoding determines the content. In order to distinguish these types in the encoding that uphold digitality, there must be some physical regularity that serves as a boundary that is upheld by the physical structure of the message. $(2013,206)$

In this definition, Halpin stresses the crucial interrelationship between encoding, content, and physical regularity or physical structures. Physical aspects, or materiality, play an essential role in this definition and undermine the idea of digitality as 'mysterious immateriality': "there must be some physical regularity", some physical substrate that has "the proper physical characteristics that uphold the physical structure of the message" (ibid.).

In the context of digital and social media, digital mysticism also used to be, and still sometimes is, expressed in what social media theorist Nathan Jurgenson calls "digital dualism" (2011, n.p.). He describes it as a fallacy that assumes a, somehow disembodied, "second self" (Turkle [1984] 2005) online. He argues that: 
social media has everything to do with the physical world and our offline lives are increasingly influenced by social media, even when logged off. We need to shed the digital dualist bias because our Facebook pages are indeed 'real life' and our offline existence is increasingly virtual. (Jurgenson 2011, n.p.)

According to Jurgenson, the danger of keeping the digital world separate from the politics, structures, and inequalities of the physical world is that it encourages the idea of the internet as an objective space that transcends social structures. One example of this is manifested in the hidden power structures of Wikipedia, when seen as a supposedly neutral, democratic space - a notion highly contested today, particularly from intersectional gender perspectives (cf. Carstensen 2013; Glott and Ghosh 2010; Herbold 2011). “Computational objectivity,” as Jurgenson also calls it, is thus a mechanism that is used to obscure structural inequalities, which often spill over into digital media contexts (2011, n.p.).

This becomes visible, for example, when the affectivity of hate speech online is dismissed by some (a position I will criticize in chapter four), notions of a rational detachment from the body are considered an asset in computing (a cultural parameter that I critique in chapter five), or when digital visual cultures are viewed from a supposedly objectifying distance (chapter six).

In contrast to digital mysticism and digital dualism, more novel scholarly attempts to combine affect theory and internet research do not only take network technologies seriously as intrinsic aspects of everyday life, but also consider the affectivity of digital media and its influence on human perceptions and activities (Hillis, Paasonen, and Petit 2015, 9).

For example, digital media theorist Jussi Parikka argues that 'digital' is "too broad a category for any specific understanding of the weird materialities of network culture that relate to movements across scales and between human and nonhuman agencies" (2015, 110). Instead, software and code are increasingly not only about machine milieus but also about their affective entanglements with bodies, 
from the abstract machines of capitalism to the concrete assemblages where we twist and turn our pelvises, knees, necks, and thumbs, sharpen our eyes, move our gaze across the screen and its surroundings, resharpen and blur - as when dealing with games, browsers, and mobile interfaces. (ibid.)

Thus, affect takes into account digitality's physical regularities, as Halpin has pointed them out above. Even more to the point, from the perspective of affect theory, the body might become (part of) this material regularity. Drawing upon digital media philosopher Mark Hansen's work (2004), affect scholar Patricia Clough argues that the digital requires the affectivity of the body, since "digitization engages bodily affect, inviting it to give information a body. Bodily affect is called to transform the unframed, disembodied, and formless into concrete embodied information intrinsically imbued with (human) meaning" (Clough 2010, 211).

For the purpose of this dissertation, I understand digitality first and foremost in its relation to affectivity. In this sense, I follow MarieLuise Angerer's assessment that, in the aftermath of Donna Haraway's work on cyborgs (Haraway 1990; Haraway 1991) ${ }^{38}$ and its continuation in the work of N. Kathrine Hayles (1999) and others, "media cannot be understood any longer as prostheses that amplify the senses" (Angerer 2015, 116). Instead, they must be seen as having a "new immersive di-

38 Donna Haraway's famous feminist figuration (Haraway 1990) suggests that, alongside the increasing sophistication of technology, boundaries between human and (bio-, informational, and otherwise-technological) machines have become blurred, and she invites a reconceptualization of troubling dualisms, such as male/female, culture/nature, civilized/primitive "all systemic to the logics and practices of domination of women, people of colour, nature, workers, animals - in short, domination of all constituted as others" (Haraway 1990, 177). The cyborg is a hybrid of materiality and imagination: On the one hand, we are always already cyborgs, be it through contraception technologies or wearing headphones, and, on the other hand, it encourages positive feminist fictionalizations that open up space for different futures, for alternatives to the rather destructive scenarios that are often prevalent in mainstream sci-fi or (cyber)discourses. 
mension, replacing our senses, making them more intense and subjective, more intimate and technical" (ibid.). In other words, for the purpose of this dissertation, I am focusing on digitality in relation to the bodily affects it engages.

In the following section, I will conclude this theoretical part by laying out my approach to digital intimacy.

\section{Digital Intimacy}

The Internet connects the entire planet. Its [sic] time we start using those connections for good.

The Digital Intimacy Intitute [sic] is dedicated to pushing web technology into the realms of love, sex, and spirit.

For the first time in history we actually have the tools to connect to every American and the rest of the planet, too!, [sic] The Web is being grossly under-used for its true purpose! It's time to stop seeing the internet as a cool way to buy your books and start seeing it as a digital love machine! 39

The Digital Intimacy Institute, which, judging by its homepage, is not so much an established institute as an amateur web initiative, was initiated in response to post-9/11 grief, according to the founder. Its homepage welcomes the user with an animated stick figure holding a stationary computer above its head that radiates hearts into the desktop's nowhere. Digital intimacy in the homepage's self-description is tied to its traditional domains of love, sex, and spirit, a slightly overoptimistic and US-centric notion of connectivity, and a refusal to limit the internet to e-commerce.

What initially captured my attention was that this homepage is the first hit when entering 'digital intimacy' in a search engine, the official address being digitalintimacy.com. I read its creator's approach as a search and yearning for a 'friendlier' world, and the hope is to find the

$39 \mathrm{http}: / /$ digitalintimacy.com/, last accessed 15/01/2016, their emphasis. 
tools to create one in digital realms: "The Internet is begging us to use it to connect this global community of compassion" (ibid.).

Then again, my conceptualization of digital intimacy, and its centrality for my work, is a rather different one. In this theoretical chapter, I have argued with Berlant that intimacy is better understood as extremely mobile processes of attachment. And indeed, such a perception does resonate with the way in which intimacy has experienced a renaissance as an analytical category in disciplines such as social sciences and feminist studies. Through this work, the inviolability of human-to-human, heteronormative, and institutionalized intimacy becomes brittle, and I see affect theory with its focus on "all of the incredible, wondrous, tragic, painful, and destructive configurations of things and bodies as temporally mediated, continuous events" (Coleman 2010,11) as a logical extension of this development. Taking this as a vantage point, I argue that intimacy is always affective, and that affect is always the prerequisite for intimacy.

From a more explicit media studies perspective, I engage with Bolter and Grusin's well-known concept of remediation (1999) to argue that intimacy is something that is always already remediated. In addition, I am following Kember and Zylinska's (2015) expansion of the concept to argue for a less human-centric view and to see 'media events' as more influenced by "technological, social, economic, geographical, and other influences of forces well beyond those controlled by the human" $(2015,9)$. I also pay special attention to how hypermediacy and (transparent) immediacy are "two interdependent aspects which can both enact and critique traditional beliefs about gender and self" (Bolter and Grusin 1999, 340).

Finally, my understanding of digitality - which in the framework of this thesis is predominantly focused on how digitality relates to affectivity - feeds into the definition of digital intimacy. In conclusion, it can be said that my conceptualization of digital intimacy oscillates between the cornerstones of intimacy, affect, remediation, and digitality. 



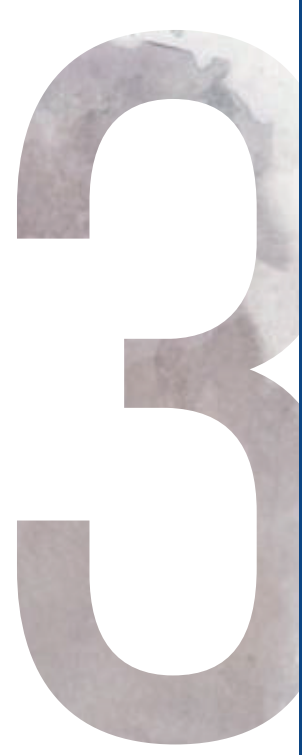

APPROACHING DIGITAL INTIMACY

METHODS AND MATERIALS 



\section{Approaching Digital Intimacy: Methods and Materials}

In this chapter, I first explain my epistemological situatedness: what are the fields and areas of research that informed my study? Next, I elaborate on how the research process led me to choose my topic and my strategic examples; then, finally, I discuss how I generated my materials, the methods I used to analyze them, and the overarching methodological framework.

\subsection{Situating the Thesis Epistemologically}

My dissertation project is located in the field of feminist cultural studies of digital media. Cultural studies, as initiated by the Birmingham Centre for Contemporary Cultural Studies in the 1960s, grew out of Marxism, structuralism, and feminism. It has always been an interdisciplinary discipline searching for new institutional forms in new national settings (Storey 1996). Studying culture thus includes a wide range of meanings; from, for instance, understanding culture as everyday life and meaning-making practices, to analyses of the ways in which power is exerted and politics are done in mundane settings, to exploring how ordinary life experiences influence identities and/or appear as mediated popular entertainment culture.

In addition, feminist cultural studies foreground the sphere within which class, gender, race, and other inequalities are negotiated (Thornham 2000). Indeed, feminist cultural theorizing and its highly diversified work on the co-constitutive relations of identity, difference, inclusion, and exclusion, has made itself relevant as an analytical tradition for all kinds of topics or phenomena (Franklin, Lury, and Stacey 2000, 6). The goal is to map social relations and their hidden power structures, as well as to uncover and recover the societal positions of neglected groups (Lister et al. 2003, 49). For these purposes, primarily 
semiotic, textual, and discourse analytic tools (studies of meaningmaking practices) are combined with social anthropological and ethnographic ones (studies of everyday life practices) (Lykke 2010, 27).

Doing this within the framework of intersectionality (as introduced by Kimberlé Crenshaw, 1989), which means taking the intersections of gender, class, age, race, ethnicity, nationality, and sexuality into account as a conceptual lens, has also become a nodal point in much of contemporary feminist cultural studies (Lykke 2010).

It is precisely this merging of everyday life and meaning-making that is crucial to my project, since I am looking at digital cultures which are part of popular culture and which influence the everyday life contexts of many people. At the same time, I hope to be able to recover the societal positions of neglected groups (e.g. by discussing the gendering of technological imaginations and the subject positions available to self-identified women online and in other digital contexts).

As science and technology studies scholar Anne Balsamo has pointed out, feminist cultural studies include a multitude of political standpoints (e.g. women of color, queer, post-colonialist) and are grounded in different intellectual traditions (e.g. poststructuralism, psychoanalysis, sociology, philosophy, anthropology, film studies, literary studies, and education) (1991, 50). She concludes that it is "impossible to define the field in terms of a dominant paradigm, and to do so would certainly not be in keeping with the open and inclusive intent of the field itself" $(1991,51)$. She therefore argues that it should be called post-disciplinary (Balsamo 1991, 50; see also Lykke 2010).

Balsamo is carving out two main strands that she believes to be dominant: one centers around matters expressed from a postcolonial or women-of-color perspective, as "questions of position, nationalist and ethnic identity, and cultural theory", and one that focuses on "the developments in feminist thinking about science, technology, and the body" (1991, 50).

Even though Balsamo was capturing the status quo at the beginning of the 1990s, I am following her rough categorization and locate my work in the second strand, as feminist thinking about science, technology, and embodied subjectivity. More particularly, I am drawing 
upon the synergy of feminist cultural studies with science and technology studies (STS) and feminist technoscience, which share the belief that science and technology are neither socially pure nor innocent in sociopolitical terms, but are instead shaped by cultural settings and entangled in societal interests. Such studies shift attention to "technoscientifically mediated processes of materialization [...] which take place between discursivity and materiality, culture and biology, subjectivity and embodiment” (Åsberg and Lykke 2010, 300). Furthermore, I also situate this dissertation in a context of media culture and digital media studies. For example, I am drawing on feminist film theory as well as on (digital) media theories such as the analytical concept of remediation (Bolter and Grusin 1999), as I have explained in my theory section.

In particular, I see affect theory and its analytical sensibilities as helpful in bringing together the approaches and analytical attitudes of feminist cultural studies with (digital) media theory in general and digital cultures in particular. Affect theory helps us to understand the role of affective attachments - for instance, affects such as desire, memory, anger, political passion, and also investments of labor and time (Hillis, Paasonen, and Petit 2015, 1) - in everyday cultural contexts. Furthermore, I see affect theory approaches as foregrounding the dimensions of the body and different materialities, which provide a way to discuss how discourses "form ligatures with pulsing flesh-and-blood creatures" (Schaefer 2013, n.p.). This is particularly important from the point of view that technologies are accessed with and through bodies, but is also crucial in order to take the affectivity and agency of other materialities, such as the digital or digital devices and their broader socio-technological networks, into account.

I thus align with Greg Goldberg's assessment that media and cultural studies are at their most astute when the theoretical and the material are engaged in close dialogue, "transgressing their boundary and revealing their mutual interdependence" $(2012,242)$. In fact, he sees this dialogue as a foundational strength of media and cultural studies, and as evident in seminal works such as Walter Benjamin's “The work of art in the age of mechanical reproduction" ([1936] 2010) or Marshall McLuhan's claim that the medium is the message ([1964] 2001). This 
conversation between theory and the material, Goldberg argues, is very much reflected or revived in the so-called affective turn $(2012,242) .4^{40}$

The turn to affect can be understood as a critique of the previous 'textual turn' and what are considered to be the limitations of textual analysis. Structuralism and poststructuralism are reviewed for their "commitment to linguistic models, (logocentric) views of language as the general framework for understanding human activity, the focus on the unconscious and the Oedipal, and an emphasis on notions of lack when thinking about desire and human activity" (Paasonen 2011a, 9). The goal is to direct attention away from representation, ideology, meaning, signification, and its "certain tyranny of the semantic at the cost of the sensory and material" (ibid.). Representationalism is thus an epistemological dualism which seeks to separate not only the researcher from her object, but also the word from the physical world and matter from meaning (Paasonen 2011a, 9). An attunement to affective cultural dynamics might remedy this.

Affect in the Deleuzian sense is what comes before the act of interpretation (Abel 2007, n.p.). It is the "ontological pre-condition for any instantiation of representation, meaning, understanding, cognition,

${ }^{40}$ However, the affective turn also has been criticized as a case "of turning away from issues of power, ideology, and politics in its embrace of the lively and the positive" (Hillis, Paasonen, and Petit 2015, 5); for example, by Clare Hemmings in her reckoning with the affective turn, in an article called "Invoking affect" (2005). One point of critique has been that "[p]ositing affect as a 'way out' requires that poststructuralist epistemology have ignored embodiment, investment and emotion. [...] [the] authors' chronologies of the past and future of critical theory need to ignore the range of poststructuralist work that does not follow this pattern" (p. 557, her emphasis). The critique is thus that Massumi (and others) are knowingly sidestepping different branches of feminist theory and, more generally, "theory written from the margins" (p. 558), and Hemmings names postcolonial and standpoint theory as examples. It is always a problem that a 'turn' to something usually implies a 'turn from' something else. In this case, as Hemmings argues, the old seems to be feminist, queer, postcolonial analysis and the new is new materialism, and there is a risk of downplaying the old. But affect researchers focus rather on sensation, intensity, materiality, and the embodied in their studies of culture and society, instead of what is criticized, for example in the 'textual turn', which is more focused on analyses of ideology, meaning and representation. 
and consciousness to emerge" and is therefore "amoral, a-representational, a-signifying” (Abel 2008, n.p.). Consequently, a focus on affect does not question what is mirrored in the act of representation, but asks about actions, performativities, matters and practices. In other words, affect in a media/cultural studies context emphasizes a switch from the meaning of images/content to how they work. The 'I', or the body affected, is not a neutral observer interpreting meaning here, but is defined through its response-ability to the forces affecting its body (Abel 2008, n.p.) in the aftermath. Goldberg argues that this change in focus challenges anthropocentrism in the social sciences and meaningor representation-centric strands in media and cultural studies by asking us to take bodily matter seriously $(2012,242)$. It is precisely this desire to take bodily matter seriously within media and cultural studies that I wish to accommodate in this thesis, and I see it as being in correspondence with recent works on affect and public emotions in the digital arena. ${ }^{4}$

Taking this overview as my epistemological position explaining the situatedness of my analytics and its relation to previous research, in the following section I will describe my analytical points of departure in more detail.

\section{Departures: How I Encountered my Material}

I am not a very geeky person when it comes to the world of computing. My experiences with coding, apart from some long-forgotten introductory coding lessons in high school, are almost exclusively limited to my visit to the coding workshop that I describe in chapter five. My excursions into the world of (micro-)blogging, tweeting, or participating in online discussions have been few and brief and, even though I do have social media accounts, my initial interest in using them for pleasure has

${ }^{41}$ For example, Paasonen 2011a; Karatzogianni and Kuntsman 2012; GardeHansen and Gorton 2013; Bensky and Fisher 2014; Hillis, Paasonen, and Petit 2015; Hillis, Paasonen, and Petit 2015; Sundén 2014; Sundén 2015a; Sundén 2015b. 
faded. On the one hand, this is because I do not like the way in which work and life, supposing that such a separation indeed can be made, are becoming increasingly mixed together in social media. On the other hand, I have become much more aware of the nasty sides and vulnerabilities that are exposed when online. However, living in a different country from most of my family and closest friends, I am intimately dependent on digital technologies to keep up with them, so I have a bit of a love/hate relationship with social media in my private life.

Based on this love/hate relationship, I am utterly fascinated by what new digital technologies can do, and what they do for us and with us, and am thus 'geeky' about their plurality and how they have become intimate aspects of many modern lives. Therefore, I accept a particular "digital mysticism", in the sense that the complexity of coding or hardware details often remain hidden behind user-friendly interfaces (van den Boomen et al. 2009, 9), and instead focus on what these changes do when they become an integral part of many people's everyday lives and are an intimate presence therein. What novel or remediated problems do these changes bring with them (digital inimicalities)? And what impulses exist to counteract these (both at a broader societal level and for feminist internet politics in particular)?

When I started with the research for this project, my main motivation was to discuss aspects of gender-related harassment and hierarchies online. One of the things that inspired me was the straightforwardly ethnographic approach of Lori Kendall, who researched communication cultures in male-dominated online forums (2002). Therefore, I was also generally interested in methodological questions about how to do online ethnographies as a developing field of research (Hine 2000; Markham and Baym 2009).

Back then (at the beginning of 2012), questions of digital racism, sexism, and forms of online discrimination and abuse were only in the process of becoming objects of academic inquiry and only partially concerned with changes characteristic to the massive expansion of social media and digital devices. From my point of view, the topic has literally exploded since then, because more and more affected people have spo- 
ken up, particularly journalists (Hess 2014; Gardiner et al. 2016), activists (Bücker 2014), and sometimes even celebrities; as, for example, when actress Jennifer Lawrence spoke up about her private accounts being hacked in order to leak private nude pictures (Prince 2014). Since then, online hate and harassment have become a much-discussed topic.

While I started to collect and archive news reportage, first-hand reports, and other forms of documentation of instances of hate and harassment online that I came across, it occurred to me that there is a grey area about what can be done about this or, considering the magnitude and complexity of the problem, at least what could be (theoretical) starting points for counter-actions.

I learned about women's programming courses while reading a digital magazine with the significant name Sister Magazine: A Journal for the Digital Lady (2012), which I started to read because, well, questions of digital participation seem to be of interest to a 'digital lady'. The magazine introduces the women's coding network Railsgirls and underlines the necessity for women and girls to contribute to technological change, in this case by learning to use the web application framework Rails. ${ }^{42}$

I was intrigued by this emphasis on the contributions of women because, in contrast to the research on online hate and harassment, initiatives such as Railsgirls are optimistic in their outlook and promote a relatively clear idea of how to achieve change. I felt that it was productive for my research to focus on such encouraging initiatives rather than working solely on causes or consequences of digital inimicalities. In this context, I also remembered that I had read about hatr.org earlier and realized that this was one of the first attempts to strategically oppose online hate and abuse. I was fascinated by its unconventional approach and queerfeminist background, and wanted to analyze its

${ }^{42}$ This web application was written in the programming language Ruby by David Heinemeier Hansson and is also called Ruby on Rails. 
tactics in order to see which of these could possibly be assembled together with the ambition to arrive at a more general intersectional feminist internet politics.

I first came into contact with ASMR when the women who produce it were described as "whispering sirens" in news reportage (Graff 2013), 43 and initially dismissed it as nothing more than an amusing internet curiosity. Only later, when I started to think more about the role of the body, multi-sensory and affective experiences during processes of digitalization, and the importance of the dissolution of boundaries such as digital/non-digital, embodied/disembodied, detached/attached for digital intimacy, did I begin to see ASMR firstly as emblematic of a changing understanding of intimacy, and secondly as an interesting contemporary example of questions of representation (that go beyond the visual) in a web 2.0 context.

In sum, I realized that these three strategic examples - hatr, the coding initiatives, and ASMR - are three examples of what it can mean to be 'a digital lady' today. For many women, such a subject positioning means being confronted with digital inimicalities like discrimination, harassment, or abuse, with questions of objectification and an increasing corporeality in the digital realm, as well as questions of digital participation in general.

\subsection{Methodological Considerations: Remixing with an Ethnographic Attitude}

In the following, I lay out how I approached my material methodologically, as the three strategic examples are ontologically quite different from each other. Therefore, I needed to carefully consider how to approach them, even though, as I have argued, they are united by constituting aspects of digital intimacy and their respective alignment against different kinds of digital inimicalities.

${ }^{43}$ This translation from German to English is mine. 
Hatr's material is displayed on a homepage, which means its domain is apparently located in the realm of the digital. Even though I will elaborate later on how it transgresses this setting when affecting bodies, the material is first and foremost generated from digitalized text. The coding initiatives, as I will argue, constitute a contemporary societal trend, which manifests itself in non-digital initiatives and communities with outlets in digital domains. ASMR is a "sensory phenomenon, in which individuals experience a tingling, static-like sensation across the scalp, back of the neck and at times further areas in response to specific triggering audio and visual stimuli" (Barratt and Davis 2015, 1), which has gained acknowledgement and popularity via the distribution channels of the internet and social media, and is usually evoked through digital video clips (in the form of the tingles or other forms of intimacy).

In sum, I am, at least initially, comparing digital texts, off-line initiatives with a somewhat pedagogical, almost equality-activist mission (and partially economic interests), and digital video clips and how women are represented therein. The degrees to which the digital is embedded varies: online hate and hatr would obviously not exist without the digital, or would take a very different form; in the coding groups, digitality is rather a pedagogical content than a framework condition; and whether ASMR could exist without being a social media trend is up for discussion (at least it would exist in a different form). How can I combine and analyze these different kinds of material?

In this context, it is useful to evoke what Donna Haraway has famously coined an 'ethnographic attitude' (1997). She used the term in her analysis of reproductive technologies and their relationship to visual technologies. She named the term in order to tie together ontologically different objects such as, in her example, the politics of self-help and women's health movements, the positions of feminist science and technology studies, and several other disciplinary and activist locations, to turn them into "critical feminist technologies for producing convincing representations of the reproduction of inequality" (1997, 22). In her take, ethnography is not only a specific procedure in anthropology: 
An 'ethnographic attitude' can be adopted within any kind of inquiry, including textual analysis. Not limited to a specific discipline, an ethnographic attitude is a mode of practical and theoretical attention, a way of remaining mindful and accountable. Such a method is not about 'taking sides' in a pre-determined way, but neither are moral and political commitments hygienically expunged. Ethnography as I understand the practice is about risks, purposes, meanings and hopes - one's own and others' - embedded in knowledge projects, including technoscientific ones. (Haraway 1997, 39)

An ethnographic attitude thus allows me to combine seemingly unlikely pairings of materials, such as different kinds of texts, artifacts, visuals, or life narratives beyond the boundaries of disciplines, in order to achieve "sustained scholarly interaction among living people in living situations, historical and contemporary, documentary and in vivo" $(1997,27)$. For my project, adopting an ethnographic attitude means combining the digital and non-digital, video material, texts and tweets, media reportage and blog entries, interview snippets and participant observation in order to create new, generative connections.

Combining such diverse kinds of material has two direct main implications for my research. Firstly, that this project has to be one that is conducted from the epistemological standpoint of situated knowledges (Haraway 1991) and, secondly, that I need to engage with contemporary approaches to internet research (as I will do below).

Situated knowledges, a feminist type of objectivity or way of seeing and knowing famously suggested by Haraway, means to be aware of the partiality of one's own analytical devices and the limits of one's perspectives, and yet to ally, preferably with the subjugated, in an openly declared way. With situated knowledges, Haraway wants to advocate a theory and practice of objectivity that privileges "contestation, deconstruction, passionate construction, webbed connections, and hope for transformation of systems of knowledge and ways of seeing" (1991, 191). In my context, this means acknowledging the situatedness of my choices and the prioritization of my strategic examples. It also means 
acknowledging the fact that not all the materials I have worked with over the years are fully visible in the analytical chapters, even though they have still contributed to the forging of my understanding of the larger phenomena studied. Finally, to base my project epistemologically on a Harawayian understanding of situated knowledges also means to acknowledge that, in this project, political commitments are not "hygienically expunged" $(1997,39)$, as becomes clear in the fact that one of my goals is to contribute to a feminist internet politics.

Thus, subscribing to an ethnographic attitude also means to be at risk - as Haraway says, "morally, politically, technically, and epistemologically" $(1997,39)$. This being at risk means to put one's own assumptions under scrutiny when meeting the other, to remain careful and responsible, and to allow the material to affect oneself and understand how one is also affecting it. In this sense, the ethnographic attitude forced me to keep an open mind during my encounters with different types of materials.

In the case of researching digital spheres of affect, or affectspheres, one is also at risk because they are characterized by "processes, practices, sensations and affects that move through bodies in ways that are difficult to see, understand and investigate" (Blackman 2012, IX). What thus becomes "necessarily risky" is the "process of mediation between the embodied and the sematic" that appear when trying to articulate the private, the intimate and the personal in writing. This is because writing about affect is, even though visceral, at the boundary with the elusive: By the time an affective intensity is named and identified, it is already gone (Hillis, Paasonen, and Petit 2015, 12).

Writing is therefore an act of mediation where bodily impressions, modulations, arousals and motions are translated in order to be brought into the representational space of the text. While much is unavoidably transformed and lost in such translation, this mediation between the sensory and the textual is nevertheless a key aim in affective forms of writing. (ibid.) 
If it is only possible to write from an own point of view, about what one has sensed, what can be said about the collective workings of affect, or affect on a more abstract level (ibid.)? One solution, according to Hillis, Paasonen and Petit, is to utilize personal and experimental forms of writing, since they "open up possibilities for bridging the gaps between the sensory and the semiotic, the personal and the collective, and for mediating the physical within the textual" (ibid.).

So is it for example not possible to determine individually who has been affected, and in what way, by receiving hate messages (or being spared from them, for that matter), in the context of hatr, or how it has affected the volunteer who was filtering it, or whether the sender was affected by the misplacement of their message. Despite this, it is possible within Ahmed's framework of affective economies, which engages with how hate travels affectively, as I will show in chapter four.

Similarly, it is risky to draw generalizable conclusions from a singular affective experience of coding workshops. It is, however, possible to understand the role of affective attachments within the coding initiatives' everyday cultural context. ASMR becomes even more elusive since it is both very public (the video clips) and a very private experience (that users usually have in the privacy of their homes), and since the ASMR response differs so much from person to person. As I explained, some experience the tingles, others a flow-like state, or they experience other forms and states of arousal or relaxation.

Thus, the riskiness here lies in the possibility that the researcher might get 'lost' in their own intensities when experiencing ASMR or, conversely, not feel it at all. But what does it mean to 'not feel it'? Personally, I do not experience the tingles as a sensation on my scalp, and some of the videos that I watched during the course of this research seemed bewildering rather than calming to me, while others were rather mesmerizing. Nevertheless, through strategies of creative writing in the thick descriptions, I try to "open up possibilities for bridging the gaps between the sensory and the semiotic," as Hillis, Paasonen and Petit put it $(2015,12)$, trying to make my own experience graspable for the reader. 
Given that affect has a tendency to move between the sensory and the semiotic, there will be a certain degree of "epistemological uncertainty" when it comes to ethnographies of digital media, since the limits of the body and of the field itself are anything but clear. I understand this as yet another sign for the degree to which digital media have become more intimate: talking about intimacy as a relationality of "much more mobile processes of attachments" (Berlant 1998, 284) means discussing things that do not always come in predictable forms.

Engaging with the affective digital also means thinking of a way to approach a body of digital material that is not solely digital, that is to acknowledge that digital cultures are not only played out in digital arenas. Such methodological considerations prompted me to discuss my take on internet research, a large, varied, and growing field of research.

Internet research is still a relatively new terrain and there are many discussions about the best ways to investigate online cultures. While digital ethnographies often focus on what is happening on the screen, social media researcher danah boyd 44 describes her approach when it comes to the investigation of (at that time) lively and fluid social networking sites like this:

Every day, I look at random MySpace profiles [...]. I interview teens from different cultural backgrounds. I talk with parents, the site's creators, and adults who use the site. I read commentary about MySpace on blogs and in the news; I listen to people talking about MySpace on the bus and at malls throughout the United States. Through my blog, others know that I'm researching MySpace, strangers send me data on a daily basis. In this way, I've begun this project in the widest way I could possibly imagine. (boyd

\footnotetext{
44 boyd intentionally writes her name in lower case. Inspired also by the work of bell hooks, who seeks to subvert grammar prescriptions and criticizes language as a construct that keeps up hierarchical power dimensions (cf. 2013), she states: "Well, we're in a digital age and the computer conveniently spaces the 'i' quite properly to make it recognizable, so i gave up on giving it such a special level of importance - it is referring to me, right? I thought an attempt at minimalizing the individualization could start at home."

(http://www.danah.org/name.html, last accessed 01/07/2016).
} 
2009, 28)

Her strategy is to assemble information from manifold sources in order to understand the social network as a continuous cultural environment. She is working with textual analysis, collecting snippets and links, and gathering ethnographic field data in order to have the widest possible outreach and then to narrow it down according to the research question and what kind of culture to focus on (e.g. early adopters, 2009, 29). 45

In a way that convinces me, boyd sets out her reasoning for the approach of starting research projects in the widest way possible by pointing out the changes in the understanding of culture in ethnographic studies. While culture has traditionally been understood as more or less bound to particular geographical spaces, this conception has been challenged by the increased mobilization of people in the context of globalization, and it has been challenged by conceptions of culture that focus on tastes, languages, interests, and so on, rather than on heritage or particular places. However, as boyd points out, "mediated technologies changed the rules entirely" (2009, 27).

Thus, it is not only often futile to ask for actual geographical locations in digital ethnographies, but digital cultures are also less frequently bound to particular media genres, or to particular online 'spaces' (like for example a particular online forum). They are not even entirely restricted to the realm of the digital: "In contemporary networked life, culture is socially proximate not geographically defined; creating boundaries by medium or genre only confuses matters" (boyd 2009, 28).

For this research, this means that, alongside the fact that the boundaries of self and others are becoming intricately entangled in new ways through digital media, I also need to take into account that the

45 Following this lead, I would like to specify that I am focusing on the culture of haters and those trying to oppose the digital hate in chapter four; on the culture of women coders and digital tech enthusiasts in chapter five; and on the subculture of ASMRtists and their fans in chapter six. 
boundaries of genres or different media, and their spatial and temporal boundaries, become blurred.

For this reason, I have already argued for the use of remediation and that I understand intimacy itself to be remediated in digital contexts. I have reasoned that the two aspects of remediation, transparent immediacy and hypermediacy (that is, firstly, the longing for or perception of mediated experiences to be authentic and, secondly, the awareness of their technologically framed contexts, two aspects that slide in and out of each other constantly), play a major role in intimacy becoming digital and the other way around. But what is needed is a method that picks up the threads at exactly this point, when social beings, digital technologies, and devices become assemblages.

Hence, I now turn to digital and social media researcher Annette Markham. Markham has developed a 'remix method' in order to capture the way in which "our understanding of the world is remixed by our engagements with social media" (Markham 2013, 65). Drawing on Kincheloe's bricolage approach (2001), Markham takes the term 'remix', which refers to "the processes and products of taking bits of cultural material and, through the process of copy/paste and collage, producing new meaning to share with others" (ibid.) and extends it to academic inquiry. While much internet research has been plagued by attempts to, so to speak, reinvent the wheel, she proposes to instead remix existing methods for analyzing media, texts, and cultural sensemaking. Remixing to her is a method "'below method', where we engage in everyday practices of sensemaking” $(2013,65)$. Therefore, her remix method focuses on aspects of research activities that are usually rendered invisible, such as playing around with different perspectives, borrowing from remote disciplines, and moving through different variations of one's work (ibid.). I agree with her that this approach to method has a range of advantages for investigating digital phenomena, which I will list below.

First of all, thinking through remix has a lot do with sense-making in everyday life, “digital or not” (2013, 70). We constantly remix digital and non-digital input to make sense of our worlds. As she puts it, remix 
is not something we do in addition to our everyday lives, it is the way we make sense of our world, by transforming the bombardment of stimuli into a seamless experience. If we take seriously the idea that everything we take to be real is a constant negotiation of relationships between people and things, and that culture is habit writ large, remix is a form of sense-making that embraces this framework. $(2013,70)$

Approaching material from this perspective thus means having situated access to particular assemblages of the digital and the non-digital. This can also be seen as an intimate and affective account (in the sense that sense-making is understood as ever-changing relationships between people and things).

Moreover, remix is a technique that is inherent to the digital cultures themselves $(2013,71)$, for example, in media convergence, digital memes or the mashing of videos, and flattens out hierarchies between different bits of information (thus attempting to avoid digital dualist thinking).

By taking "existing elements to connect the familiar with the unfamiliar, or the original with the remixed" $(2013,70)$, remix is in addition able to generate complex analyses of complex phenomena. Transferring this insight into an academic tool for thinking about qualitative, interpretative research practice, she argues, offers

a lens through which we may be able to better grapple with the complexity of social contexts characterized by ubiquitous Internet, always-connected mobile devices, dense global communication networks, fragments of information flow, and temporal and ad hoc community formations. $(2013,65)$

To summarize, remix as a methodological framework allows me to embrace the diversity of both my material and my methods and to grapple with their complexity. In the following, I lay out how I use this framework in more detail. 


\section{Generate, Play, Borrow, Move, and Interrogate}

Markham suggests five elements of remix, which appear at the beginning of each research process and which she tries to make visible. Since they have been instructive for my approach, I would like to detail them here:

What she terms 'generate' refers to the process of generating data, how data is gathered and 'layered' and does not sit 'out there' waiting to be approached, but has to be decisively collected, and thus produced. We transform data by classifying it, layering it differently each time, and creating a new perspective as we go. "Reflecting on these and other practices, we can see that inquiry is not only about simplifying and narrowing, but generating layers upon layers of informational units that influence our interpretations" $(2013,74)$. This is particularly important in a digitalized context, in which the material is not lying "on one's desk" (2013, 74-75) and thus an aura of immateriality.

'Play' is used here in the sense of playing games or playing an instrument: both as creative play and as mastery, and combining both in order to create something new (even though the tools might be well known). This is thus a way to work creatively, to find new connections and solutions, but also to allow oneself to try and fail using different methods. It is precisely this playing which can become "a critical turning point for research design that resonates better with contexts of flow, analysis that moves with or into these flows rather than abstracting and isolating objects arbitrarily and artificially" $(2013,76)$.

'Borrowing', she argues, is already inherent in a lot of academic work: borrowing the tools of academic writing and sampling strategies or concepts is an indispensable part of academic investigations. This includes borrowing from non-academic disciplines and using non-academic material (2013, 66-67). As Markham puts it:

When we consider the way in which people use and relate to technologies for communication, the variation is endless. Borrowing approaches, perspectives, and techniques from not only outside one's discipline but from outside the 
academy seems not only natural but essential to figuring out creative ways to grapple with these contexts. $(2013,77)$

To 'move' as a thinking device encourages the researcher to follow streams and flows, "silences, gaps and absences" $(2013,77)$.

Constantly 'interrogating', finally, is a necessity in order to contextualize pieces of culture in previously unthought contexts, in a steady stream of questioning: "everywhere we see remixed production of culture, we know we are witnessing the outcome of a process of reflexive interrogation" $(2013,77)$. This happens, for instance, in my earlier example of the socialist fraternal kiss. The picture displayed on the Berlin Wall indicates a critical interrogation of the GDR, and the remixing of it many years later, when an unknown street artist writes 'Homophobia in Russia' on Brezhnev's temple, means a further interrogation by remixing what is there with a new political discourse.

In the following, I will describe my material and how I approached it while considering these five factors of methodological reflection: In what ways have I generated, played, borrowed, moved, and interrogated?

\section{Approaching Hatr}

My approach to analyzing hatr.org's archived hateful messages is pretty straightforward. I entered their homepage, read through the comments that have been re-posted there, and copied them into a word document. It is thus a textual analysis of a particular digital media genre, the genre of comments in public commenting sections (usually found under blog articles and news articles or variations thereof). I generated my material from the 21 most recent comments, which is the number of messages that were displayed on the first page. I also scrolled through many of the following pages, only to see that the styles and forms of insults, and also at times the wording, were fairly repetitive. I therefore considered the 21 entries on the first pages to be representative of the material at hand. 
My research interest in the case of hatr is not to analyze isolated hateful messages per se, but to understand how hate travels and how, in social imaginaries and practices, it sticks to particular bodies and not others (Ahmed 2004). I therefore refrained from simply re-producing the messages in carbon copy. To do so would not generate anything particularly new or productive for this analysis. However, the more playful solution that I found, which also keeps the material present and open for interpretation, was to write a short summary of these messages, pointing out what kind of insults were used, which bodily or identity markers were attacked, and, where possible, which discourses were appealed to therein.

In other words, I remixed the fragments of information flow into a text that allows the recognition of patterns, in order to interrogate them. The moving aspect here is that I followed the 'travels' of 'sticky signs' in 'affective economies of hate' into 'a place of no place', borrowing conceptual frameworks from Sara Ahmed and Judith Butler respectively and bringing them into a digital media context (2004; 1997).

\section{Approaching Women's Coding Initiatives}

The empirical material from which my analysis of the coding initiatives for women* is generated stems from motifs used in the documentary movie CodeGirl (Chilcott 2015), from historical material, i.e. re-discovered histories of the relationship between women and coding, on promotional material and mission statements from seven different women*-centered coding initiatives (as published on their homepages or expressed in interviews), and contains fieldwork material from a participant-observer perspective that is informed by an ethnographic attitude and also contains snippets of interview material.

In this way, I combine multiple layers of this cultural phenomenon, which follow a thread from past to present, from the digital to the non-digital, and from intimate to distant (in the sense that I argue that the relationship of women to coding has been construed as a 'back and forth'). Remix, Markham argues, "relies on experimenting with various 
combinations of elements so as to produce something meaningful" $(2013,77)$. In this vein, I see this layering of material as a gradual remixing process.

Firstly, I learned about the coding initiatives from digital media, then I engaged with historical figures and facts, and finally, almost accidently and driven by curiosity, I ended up participating in one such workshop myself. ${ }^{46}$ In this way, I was playfully following a particular flow, as Markham puts it.

It could also be said that I am moving from the large scale (women's coding initiatives as contemporary cultural phenomenon, as promoted, for example, in CodeGirl) to the small scale (the personalized experience of taking part in a workshop myself), while, as a step in-between, evoking historical predecessors (historical women programmers). I am thus borrowing from different domains of cultural analysis (i.e. from pop culture, ethnography, and history), in order to interrogate the phenomenon from a novel perspective.

In order to preserve the anonymity of all the people involved in the workshop experience, I use pseudonyms instead of the real names of all actors. The organization that provided the workshop in which I took part, I nicknamed HackerWomen. This pseudonym could well have been the actual name of the initiative because it catches the flavor of the original organization's name. The workshop in which I participated started at nine a.m. and lasted until four p.m., and took place in a local but internationally successful e-business's urban, loft-style offices, which were available to be used by HackerWomen at weekends. I had to pay a manageable participation fee beforehand, and the goal of the day was to create a visually pleasing personal homepage including pictures, embedded social media buttons, and some other modern effects, using CSS and HTML.47 This workshop was categorized as beginner's

46 I was traveling in North America in June 2013 when I noticed that a workshop was planned in a nearby city and spontaneously decided to change my route and participate in the workshop. To my knowledge, comparable initiatives did not exist at that time in Sweden, where I am currently based. 47 Or Cascading Style Sheets and HyperText Markup Language. HTML is a markup language (ML), which means basically that it is adding structure to 
level, for which no previous knowledge was needed. The interviews were unstructured and took place with two young women during our joint lunch break. In fact, it was an impromptu conversation, which turned out to be quite informative for my research. My opening question was: What are the reasons and motivations to take a (likely to be) all-women's programming course? The conversation was not taped, and I obtained permission from both women to reproduce particular wordings and comments that they agreed upon, as they are used in chapter three.

During the workshop, I furthermore saw myself as a participantobserver. This means that I was constantly shifting between observer and participant, zooming in and zooming out, switching from the closehaptic (keyboard, screens, instructions, commands) into the more general surroundings (atmosphere and social interactions).

The fieldnotes upon which I am drawing in this chapter are fieldnotes and headnotes (Sanjek 1990) at the same time, in the sense that I wrote down keywords and short phrases during the event (since I was busy working on my homepage and following the teacher's instructions), and developed them 'from the head', that is from fresh memory, in the aftermath of the workshop.

\section{Approaching ASMR}

My main methodological device in chapter six is Clifford Geertz' famous ethnographic method of 'thick descriptions' (1973). This approach describes culture and human behavior with more detail, meaning, and context, including interpretations and comments. This is opposed to "thin description" (Geertz 1973, 7), which is merely a factual description without any interpretation of meaning or significance. Thick description can be seen as an interpretive approach to better understand the meanings behind behavior and culture, and to do so it is necessary to include context and interpretation, so that an outsider to

text or describing a document. CSS is similar; it is a way to style/decorate HTML documents. 
the culture can also understand the meaning. Providing a thick description allows others to understand not only what happened, but also, for example, what was expressed and why. This can aid in the transferability of conclusions drawn from observations to other phenomena and contexts, and I consider it crucial for capturing the affective nuances that I want to present within this thesis.

This also means that I not only attempt to describe selected videos in a detailed and vivid manner, to give an indication of their multi-sensory attempts at affective stimulation, but I also trace their oscillations into wider social and cultural contexts. I believe that this is important because I do not want to discuss only what ASMRtists do on YouTube but also the broader cultural universe of its makers and receivers, its affective attraction, its reception by mainstream media, and what all this might tell us about intimacy and relational life in a networked society. The question is thus not so much who the people producing and watching these videos are, but how they affect viewers and confuse societal ideas of engaging intimately with the digital, the machine, and the other.

This means that, for chapter six, I generated my material predominantly through thick descriptions of the ASMR video clips. I watched approximately twenty to thirty different videos from start to finish, while admittedly occasionally skipping very repetitive or uneventful parts. The videos that I chose to discuss closely in this thesis I watched several times each, frequently pausing the streaming in order to take notes and to consider how pictures, sounds, setup, and narratives reverberate with each other and with me. Additionally, I skimmed through at least fifty more videos to get a feeling for the different subgenres, as I introduce them in the ASMR chapter.

Watching ASMR videos can also lead to being seduced by them. Many of the performers try to take viewers by the hand and make them forget their everyday struggles, up to a point where, for some, the boundaries between digital and non-digital, self and other, being touched and not being touched, become indistinct. As a playful attempt to illustrate this nuance and to question these boundaries, I decided in 
my descriptions to stylistically highlight passages in which this happens in italics. This means that in the descriptions of acts in ASMR videos I write that they are actually doing it, for example 'ASMRstarlet reaches out to touch the viewer' instead of more clumsy paraphrases such as 'ASMRstarlet leans towards the camera and acts as though she could reach through the screen to touch the viewer'. I consider this an attempt to illustrate how the actions performed in the videos might affect viewers. It is thus an attempt to sensitize the reader to how some viewers - those who are particularly strongly affected - might experience the videos as being accompanied by physical reactions (e.g., that they feel touched by the brush when VeniVidiVulpes reaches out beyond the screen).

I chose the videos that I discuss by following a snowball scheme: Since I first learned about ASMR through news reportage, I checked out the video clips of the most famous starlets (that were discussed in the reportage) first and explored their repertoires, and later moved on guided by YouTube's algorithms of clips that were most popular and recommended based on my evolving viewing habits. I thus allowed the agency of these algorithms to 'drag me along'.

While watching several videos by singular artists, I furthermore was astonished by the range of different ASMR subgenres that some of them cover, and consequently became more sensitized to videos that break the patterns typical of ASMR videos and was particularly moved along by those (for example, by Heather Feather's performance of science girl, which I discuss towards the end of chapter six; as I will argue, it breaks the pattern of typical ASMR narratives).

This focus on the stage personas of the starlets of ASMR confirmed me in my decision not to alter their performer names in my writing. It can be safely assumed that they do not mind the recognition of their work; indeed, many of them promote it when giving interviews to newspapers (cf. Cheadle 2013) or making appearances on TV (cf. Hockridge 2013), and openly relay their stage names on social media to promote their work. 
Furthermore, I generated material predominantly through other forms of digital information: comments from YouTube comment sections (where ASMR fans express their thoughts and feelings), digital news reportage, and ASMR community bulletin boards (in which ASMR users share their stories, upload and exchange videos, and define what ASMR means to them).

Relying on online information to research ASMR is not simply crucial for the sake of a dissertation on online cultures, but because information on this phenomenon can scarcely be found elsewhere. ASMR is a phenomenon that was both invented and 'discovered' through social media (Abbruzzese 2015), and is almost exclusively popularized, analyzed, and self-diagnosed within social media contexts. For this reason, and due to the relative novelty of the phenomenon (the term was coined in 2010), there have been few academic investigations into the topic during the time period of my research. ${ }^{8}$ Therefore, to investigate ASMR culture means to follow its traces click by click, from video clip to video clip, and from link to link.

Lastly, I borrow from a wide array of earlier domains of academic inquiry; for example, I draw on classical feminist film theory and pick up on earlier discussions about the role of the camgirl that took place at the end of the 1990s, in order to remix all these elements into a critical interrogation of the ASMRtists' representation, subject positionings and affective intensities.

But first, I turn to much less friendly digital domains, the world of online hate and harassment, in my first empirical chapter.

${ }^{48}$ Some examples are: Lochte 2013; Sihvonen and Tiainen 2014; Gallagher 2015; Barratt and Davis 2015. 


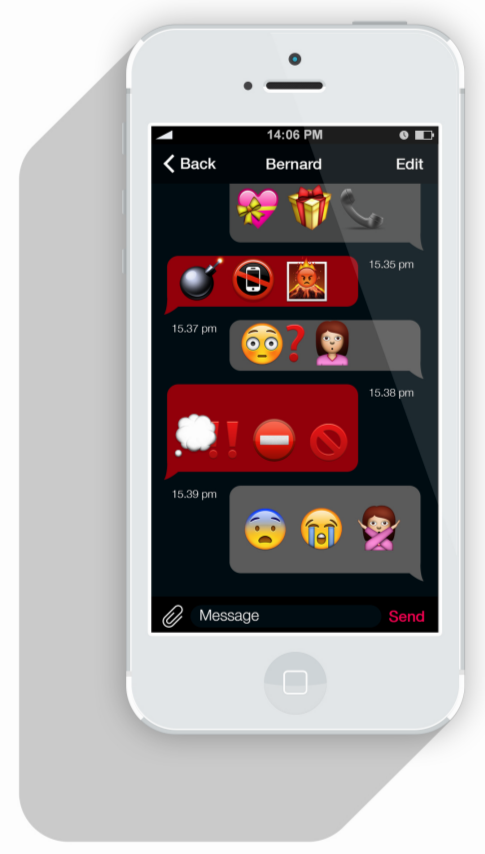

HATR.ORG 



\section{Hatr.org: An Initiative against Online Hate}

This chapter is set out to explore gender-based and intersectional forms of hate and harassment online, and inspects feminist and other digital activist initiatives to counter these forms of digital inimicalities and intimidations ${ }^{49}$. Setting the issue into a framework of past and current digital feminist activisms, I introduce the anti-trolling platform hatr.org and their strategies of displacing and relaying hate. Against the backdrop of Sara Ahmed's 'affective economies' (2004), I analyze how hateful messages are affective and intimate and evaluate hatr.org's strategy to disrupt this affective economy as a potential digital political tool.

\subsection{Background: Feminist Digital Activism as Cultural Phenomenon}

The volume Cyberfeminism 2.o, edited by Radhika Gajjala and Yeon $\mathrm{Ju} \mathrm{Oh}$, opened with the question: "Where have all the cyberfeminists gone?" (2012). Indeed, this question is intriguing: what happened to the feminist communities, artists and academic streams that discussed the internet, digital media and new technologies and their relation to society from a feminist perspective during the 1990s?50 How do their

49 This empirical chapter is inspired by my recent journal article "From \#aufschrei to hatr.org: Digital-Material Entanglements in the Context of German Digital Feminist Activisms" in Feminist Media Studies (Vol. 16, No.1, 2015).

${ }^{50}$ Susanna Paasonen has usefully provided a rough categorization of different meanings for cyberfeminism (2011b). It can stand broadly for a feminist analysis of human-machine relations, as prevalent, for example, in the work of Rosi Braidotti (2003), Donna Haraway (1990), and Sadie Plant (1997). A second branch is concerned with the application of feminist thought to cyberculture, and its specific technologies and practices, which often still have a sci-fi aura around them: cybernetics (1999), artificial life (2003) and the 
approaches to dissolving sex and gender with the help of new technologies and to linking bodies and machines translate into the context of web 2.0?

Gajjala and Oh's collection explores "what it means to be cyberfeminist now, more than a decade after feminists burst forth on the Internet scene to demand material access and social intervention both online and offline" $(2012,1)$. Here, the reader can gain an insight into what cyberfeminism might mean today: engagements with feminist narratives of empowerment in health 2.0 discourses (Levina 2012); explorations of girls' and women's experiences in (male-dominated) gaming cultures and online communities (Kubik 2012; Downey 2012; Beyer 2012) or feminist presences in the blogosphere (Angelone 2012). In other words, as academic blogger Nicole Shephard writes, taking up Oh and Gajjala's opening question:

They [the cyberfeminists, H.S.] haven't gone at all. In an environment where a comfortable online/offline dichotomy becomes increasingly difficult to maintain, feminisms are plenty and being a feminist online can take as many forms as offline. From this perspective, cyberfeminism has diversified beyond being traceable and cyberfeminists have gone everywhere and nowhere in particular. (Shephard 2013)

In other words, even though contemporary brands of cyberfeminism are untraceable in their complexity, a strong feminist participatory culture through different kinds of digital media has evolved over the past two decades (Drüeke and Zobl 2012). However, cyberfeminism has become a rather seldom-used label for feminist engagements with and

relationship between nature and culture as inseparable natureculture (Haraway 1991). As a third thread, Paasonen identifies cyberfeminisms that are more engaged with digital media and its forms of communication. Academically, it is probably this one that is most often subsumed within feminist cultural studies of digital media that investigate the interconnections of gender, embodiment, and technology (or sometimes also called feminist Internet research, Paasonen 2011b, 341). 
through digital media. ${ }^{11}$ Instead, a plethora of names is used. Whereas Gajjala and Oh suggested the upgrade to 2.0 in the title of their book, others speak of pop-feminism, DIY feminism (Drüeke and Zobl 2012), networked feminism, or even "fourth wave" feminism (Knappe and Lang 2014, 364). All of these terminologies emphasize different aspects of these feminisms: digitality, the ability to encourage networking, a focus on technologies and web-based mobilization, DIY aesthetics or a perceived progression (in the wave terminology or in relation to cyberfeminism). So what are examples of feminist digital activism today?

The feminist blogosphere, for example, has transformed feminist conversations and revived older styles and topics of feminist activism. Pop cultural outlets such as jezebel.com, maedchenmannschaft.net or feminist micro-blogging sites on tumblr are known as easy, mundane, and often fun ways to engage with feminist topics in everyday life. At the same time, these kinds of online activism do not only remain on the screen: New Yorker columnist Emily Nussbaum describes her experiences with the Slutwalks in New York: "Even as we march, it is being tweeted and filmed and Tumblr'd, a way of alerting the press and a way of bypassing the press. I am surrounded by the same bloggers I've been reading for weeks" (2011, n.p.). Here, the online and the on-street activism become parallel and equally important. Or as Maria Stehle puts it, "large-scale feminist activism today depends on popular culture in the form of digital culture, becoming as much a consumer-based participatory event as a political action" (2014, n.p.).

Activists raise awareness, connect with each other, and organize events online to mobilize support for on-street activism, performances, and art projects that are predominantly acted out non-digitally, but at the same time are accompanied and documented by digital media and thus intimately entangled with them. Events like the Slutwalks or Pussy Riot solidarity events have a 'bodies on the street' approach, with body-politics and DIY aesthetics at their heart, but the viral spreading

${ }^{51}$ One of the reasons for this might be that the original use of the term cyberspace was often to describe a disembodied digital parallel world in the spirit of William Gibson's cyberpunk writing, which hardly captures the everyday life experience with digital media of many people (Paasonen 2011b). 
of their messages through social media has become an indispensable part of such activism (Smith-Prei and Stehle 2016). Without this support, their outreach to classic mainstream media seems questionable: as Nussbaum mentions in the quote above, social digital media like Tumblr or Twitter are used to strategically bypass or alert the press, thus allowing participants to exercise some degree of control over the news coverage of these events.

Aside from the blogosphere, some of the best-known examples of recent feminist engagements online are diverse Twitter campaigns. \#YesAllWomen (originating in the USA), the \#EverydaySexismProject (originating in the UK) and the German \#aufschrei ('\#outcry') all have the same strategy, enabled by the microblogging network's hashtag functionality: users share glimpses of their lives (in 140 characters or less), to report on their experiences of everyday sexism, to declare solidarity with other affected persons, and to spark thoughtful and critical conversations about inequality and social change.

They have had success: the 2013 \#aufschrei campaign, for instance, has been described as causing a "Diskurswandel," a change in the discourse in Germany (Bücker 2014). It was the first time in years that a feminist topic had caused significant waves in the mainstream media, and it did so by uniting similar experiences under one hashtag, thereby bundling them together and eventually revealing patterns that touched thousands of people's (mainly women's) lives.

The hashtag was used more than 58 ooo times in the first two weeks (Stokowski 2014), mostly telling of discrimination in the workplace, catcalling on the street, and sexual abuse or objectification in such an abundance that it could not be ignored by the public. Questions of whether Germany has a sexism problem or where harassment starts and what to do about it became part of the public agenda. As journalist Margarete Stokowski points out, hashtags usually stay where they originate-on the Internet. But this time, an aufschrei hashtag and its social baggage managed to transgress the on/offline boundary, illustrating that there is no rigid line between 'real life' and the Internet, but that there are only affective stories of affected people. In this way, " $[w]$ ith one hashtag collective experience, these protests have begun to 
reestablish the ground for a collective feminist politics beyond the realm of the self-styled individual of neoliberalism", as gender and media scholar Hester Baer argues (2014, n.p.). In other words, digital feminist activisms today are a not-to-be-dismissed foundation for contemporary feminist social movements.

In the introductory chapter I have traced different digital inimicalities that aggravate female digital citizens' lives: Online harassment that is disproportionately often aimed at women, male-dominated techno-cultures in which women are still underrepresented, and questions of representation in visual digital cultures. Of these, online harassment is the topic that is currently receiving the most attention, and it has become a pressing issue for feminist writers and activists.

Different net activists, digital feminists, and female writers have recently begun to speak out about the (oftentimes sexualized) insults and threats with which they are confronted in their daily digital lives (cf. Schmidt 2011; Bates 2013; Eickelmann 2014; Bücker 2014). Particularly in the comments section of weblogs or online newspapers, trolls try to provoke reactions with sexist, racist, homophobic or transphobic comments, to suppress feminist and other critical opinions-comments that even utilize threats of violence and murder to intimidate the writers and activists (Carstensen 2013, 122).

In 2014, the awareness of this problem increased internationally (Marcotte 2014), and several initiatives to tackle problems of online harassment now exist. For example, the nonprofit organization Women, Action \& the Media has negotiated with Twitter to implement new reporting procedures to reduce gender-based harassment (Friedman 2014), and the Fembot Collective, an international collective of feminist media activists, artists, producers, and scholars is setting up a Fembot toolkit in which "strategies for coping and resistance" are shared online (Fembot Collective 2014).52 In other words, alongside

$5^{2}$ For example, they offer advice on how to protect oneself from doxxing (the researching and broadcasting of private and identifiable information by enemies), how to use Tor (software enabling anonymous information), and other forms of sharing experiences and strategies (http://fembotcollective.org/blog/2014/11/o7/the-fembot-toolkit/, last accessed 01/07/2016). 
missions such as political critique, awareness raising or community building, another arm of digital feminism is concerned - perforce with inimical communication cultures and is seeking strategies to change the status quo. While the offline discussion often revolves around legal measures and pleas to change the law to protect victims of hateful attacks online, other feminist writers and activists are seeking more creative approaches to counteract online harassment.

This is where an initiative such as hatr.org comes in.

\section{Introducing Hatr}

On attempting to open the homepage of hatr.org, the user is shielded off from entering immediately by a 'trigger warning'53:

The requested site contains blocked hate comments from, amongst others, feminist and anti-racist blogs. Its content can trigger strongly, that means it can trigger traumatic memories and fear. It is hard to bear.

This content warning refers specifically, but not exclusively, to

- Explicit descriptions of (sexualized) violence and threats of violence

53 In the framework of this thesis, I do not want to go too deep into the ongoing and heated debate about trigger warnings. It is only good to know that, as Katharina Kyrölä has pointed out (2015), there are basically two camps: those who think that triggers as 'warnings' before texts with sensitive and potentially disturbing, or trauma-triggering are a good idea to create 'safe spaces' (cf. Johnston 2014). And people who believe that this techniques misunderstands how trauma works, that this is a neoliberal self-centered practices where people want to close their eyes before realities (cf. Freeman et al. 2014). In the end, Kyrölä argues for the pedagogical value of sometimes feeling "really, really bad" $(2015,142)$. For hatr.org, I would argue the trigger warning is less controversial because it is obvious that the comments have little artistic value and are intended to hurt and to disturb people. In other words, independent of what camp one falls in, the trigger warning in this particular example seems feasible because the question is not if the displayed material is offensive but rather how. I thus do not consider the 'trigger warning discussion' as central for hatr.org. 
- Sexism, racism, ableism, anti-Semitism, homophobia, lookism etc. in the form of insults and verbal elaborations

- Extremely violent language

Hatr.org documents the hate net activists are exposed to. Before continuing, please consider whether you want to confront yourself with this. 54

This trigger warning is followed by two checkboxes, one asking if the trigger warning has been understood and if the user wants to continue, the other offering to enable the trigger warning for a certain time period for the user's IP address (so that frequent users don't have to click the box each time). But what is hatr.org about and why is there a need for a trigger warning? What is its strategy and purpose?

Developed by queerfeminist bloggers and their supporters, the site's concept was adopted from so-called 'mommy blogger' 55 Heather B. Armstrong, whose famous US blog received large amounts of hate mail and comments. As a resistance strategy, she started the "monetizing the hate" 56 webpage, on which she collects hateful comments that she does not want on her blog. Advertising on this outlet monetizes the nasty comments. ${ }^{57}$

Hatr.org has adopted this idea. With an extension for the weblog software Wordpress, comments posted under the entries of the participating blogs are redirected to hatr.org, where they are checked for trolling or abusive language (Reinsberg 2013). Then, trolling and hateful comments are re-distributed to hatr.org, where they are published

54 This translation from German to English is mine (http://hatr.org/, last accessed 08/05/2016).

55 'Mommyblogger' has become a subgenre of predominantly female writers who focus mainly on parenting and housekeeping issues in their blog, often during parental leave or while working from home. While this term is often used in a derogatory way to describe 'light-hearted' contents, some Mommybloggers, like Armstrong, have made a considerable fortune with this. ${ }^{6} \mathrm{http} / /$ dooce.com/hate/, last accessed 19/04/2015. Apparently, much of the hate mail relates to Armstrong's split with the religious community in which she grew up.

57 Armstrong does not specify how she uses the money. 
out of context (thus not under the posts where the hateful senders wanted to place them).

The filtering and redistributing of comments is done by hatr.org helpers/volunteers, so the writer of the blog entry at whom the hateful comment is aimed is not directly confronted with it. The service is in closed beta version, but currently around sixty, mainly queerfeminist and antiracist, blogs use it. In addition, there are few advertising banners on the page to generate more money, and hatr.org also has a fan shop for supporters of the project. ${ }^{8}$ The income will be donated to charity projects supported by the participating websites, but in its current state, this is only an idea-the monetizing has not yet been realized. 59

In this way, it can be said that hatr.org's strategy is twofold: firstly, it is raising money for charitable purposes and secondly it is raising awareness about the amount of hate with which feminist and other progressive net activists are confronted. This is particularly important since awareness of online harassment and its intersectional dimensions when it comes to the question of who is affected and how is still in its infancy. It is a difficult and complex topic without quick fixes, particular since censorship and data retention are controversial and ambivalent options.

Hatr.org's mission seems to be manifested in the platform's logo, which is a pink heart with a scribbled ' $h$ ' within: giving love to the bloggers by sparing them from hate. I was intrigued about analyzing hatr.org because I see it as an initiative that seeks to react to the problem of online harassment in a 'different' way, in the sense that it is not only reactive but also generative. Keeping my overall research question

${ }^{8}$ http://yetzt.spreadshirt.de/hatr-C194182, last accessed o8/05/2016. 59 On hatr.org it says in the FAQ: "On hatr.org, advertisement shall be placed. After all, we do not only want to put the trolls on the spot, but monetize them cold-bloodedly. We want to donate the earned money to cool projects. When the time has come, we will turn to the hatr.org community and ask for suggestions." http://hatr.org/about\#was-passiert-mit-dem-geld, last accessed 24/06/2015. 
about novel ways to counteract inimical digital communication cultures in mind, I am curious to explore hatr.org's strategy further. How exactly is hate speech defused here and how can this strategy be useful in terms of thinking about feminist positionings and approaches to online harassment?

\section{Hateful Text}

I do not wish to reproduce the hateful messages in carbon copy within the framework of this thesis. This is because I am not focusing on the content of the hurtful messages, but on what hatr does with them. However, I have accessed hatr.org several times and will give a sample of what one can expect to find on this anti-online harassment platform.

Entering the site on $8^{\text {th }}$ May 2015, I find that the landing page after the trigger warning displays 21 messages that have been filtered out as inimical and harmful by the hatr.org team. In this sample, all the offenses listed in the trigger warning (and combinations thereof) can be found:

One comment accuses the blogger of "mental masturbation" and being part of a "fringe group with an entrenched view of the world that has settled down in its mental narrowness". Another connects discourses of migration and migrants with the metaphor of flooding and relates immigrants to crimes such as burglary and rape. One comment tells the blogger to "shut up and leave the country". Several employ sexist slurs such as "bitch" and call the bloggers prostitutes. Two contain murder threats (murder by shooting), one threatens rape against the next random woman on the street. Two are more indirect wishes for the bloggers to die (e.g. by wishing assisted suicide on them). One is fat-shaming by comparing the author to a whale, another is a direct appeal to ridicule and hate trans people. Several call the writers incompetent or simply stupid, also through the use of exaggerated, ironically empathic sentences. One argues for a convicted right-wing terrorist's innocence. 
As mentioned earlier, the hateful comments are decontextualized, in the sense that they do not appear under the original blog post. It is nevertheless clear that, whatever the blogger wrote in their original post, the comments collected here are not critical-constructive discussion contributions but are employing discrediting identity markers such as those of gender, sexuality, race, and so on. The messages often aim to hit the most intimate areas on purpose: "the local, the microlevel, the private and embodied levels, and on levels that involve the psyche in one way or the other" (Wilson 2016, 249).

The tendencies displayed in this small sample reflect the finding of the Guardian's published study of types of online harassment (Gardiner et al. 2016). Female, LGBTI and non-white positions and writers are most likely to be attacked with hateful comments. Hate speech aims to affect the intimate, in the present sample in terms of one's sexuality, bodily autonomy, corporeality, intellect or assumed character traits. These attempts are affective in the sense that they work on the body in similar ways to physical aggression. The boundary between on- and offline insults becomes blurred. Activist and writer Theresa Bücker describes explains:

As a person who has grown up with an internet connection, the distinction between online and offline no longer exists. There is no 'real life' and no purely virtual life. Everybody only has one life. For activists, that means, firstly, that social engagement on the internet is real: It means effort and endeavor. Secondly, activists experience reactions to their engagements immediately, and they cause emotions. Whether it be a sense of achievement, missing appreciation, or violent trespasses-all of what activists experience in the course of their engagement on the net is real and affects them. (Bücker 2014, 118)60

${ }^{60}$ This translation from German to English is mine. 
This situating as a feminist activist online was written retrospectively by Bücker about her engagement with feminist online spheres and activisms, reflecting on uplifting experiences but also on those involving hate speech and online harassment in the context of anti-feminist backlashes. She describes how the internet has become a place for likeminded people to interconnect and build communities, but also how it is a place of a "wounded private sphere" $(2014,117)$, in which there is no "protective Doppelgänger" for a subject, but in which she is more open, more exposed, and more vulnerable instead (ibid.). This means that digital activist work is real work and has real affects and effects on physical and emotional resources: "There is no 'real life' and no purely virtual life" (ibid.). While this insight might be a given for many people, there is still a tendency to downplay online activities as 'not real', online insults as not real insults, online activism as not real activism, and so on.

But as social media theorist Jurgenson points out, in the context of online harassment there is "one person [who] is feeling the reality of the Internet very viscerally: the person who is being threatened" (quoted in: Hess 2014, n.p.). This sense of the internet's reality affects the person being threatened much more directly than it affects the hate speaker, who will usually not act on their threating words, and it affects the addressee much more than a potential police investigator who is not trained or experienced in handling digital threats.

Therefore, I believe it is necessary to discuss hate speech as affective and visceral, in order to contribute to an understanding of hate speech online as something tangible, as an object that one can tackle. In this fashion, I am following Judith Butler's assessment that

[t]o claim that language injures or [...] that 'words wound' is to combine linguistic and physical vocabularies. The use of a term such as 'wound' suggests that language can act in ways that parallel the infliction of physical pain and injury. (Butler 1997, 4) 
One way to think about how signs and language affect bodies has been provided by Sara Ahmed and her framework of affective economies, in which signs can become 'sticky' and in this way shape individual and collective bodies. In the following section, I will briefly describe Ahmed's approach, because it helps me to understand how particular online signs are becoming 'sticky' and how they attach themselves to certain bodies in the current sample of the hatr.org page.

\subsection{Affective Economies of Hate}

In her seminal The Cultural Politics of Emotion (2004), feminist scholar Sara Ahmed investigates the relationship between emotions, language, and bodies, and understands emotions as cultural practices instead of psychological states. She argues that emotions can become material rhetoric and have affective power. Following Spinoza, in her model of affective economies "all actions are reactions, in the sense that what we do is shaped by the contact we have with others" (Ahmed 2004, 4). In this way, emotions are understood to shape the surfaces of bodies, they leave a "mark or trace" upon or within them $(2004,6)$.

This is, argues Ahmed, why there is a 'press' in the word 'impression': "So not only do I have an impression of others, but they also leave me with an impression; they impress me, and impress upon me" (2004, 6). Taking this insight to a broader societal level, she argues that these movements and shapings can lead to collective politics and social alliances. That is, when emotions become cultural practices, value is ascribed to some bodies and not to others and the latter become the 'others'. To put it differently, in affective economies some bodies are aligned with a particular group (for example 'us', 'the nation') while others are marginalized and excluded. 


\section{'Floods' as Sticky Signs}

How this happens can be illustrated with one example from the 21comment-strong sample of hatr.org. A commenter accuses "the people's elected representatives" of "willfully flooding their ancestral people with humans of a contrary religion, culture and traditions", describes immigrants or refugees as bringing a multitude of crimes with them, and rants about them not being useful to the German economy because they "have an IQ below ours". ${ }^{61}$ Without context, it can be assumed that this comment has been posted in relation to the ongoing refugee crisis or some other context that involves migration issues. With only a few words, the hater here has created an exclusionary community ('the ancestral people'), and a group of 'others' who cannot share a common ground because everything about them is simply 'contrary'. The hater connected them to stupidity and criminality, all in comparison to an imagined 'us'.

The flood metaphor is well established and often used in reactionary discourses on migration, refugees and asylum. It has, for example, been used and academically criticized in migration discourses from 1990 Sermany. It is reminiscent of Klaus Theweleit's analysis of water, flowing, and streams in texts about warfare and is used to confer a feeling of threat, of being overwhelmed and overpowered, of chaos and uncontrollability (see Shariatmadari 2015 for a critique of the use of these 'toxic metaphors' in current media reportage). ${ }^{62}$

Ahmed traces the appearance and circulation of this metaphor in similar political statements in an English context in the early 2000 .

\footnotetext{
61 This translation from German to English is mine.

62 With the help of an extensive analysis of diverse texts, such as private letters, (auto-)biographies, news reportage, and art works about and by German Freikorps soldiers, Theweleit in Male Fantasies (1987) managed to trace back an overuse of water metaphors to describe the threat of the 'red armies'. According to Theweleit, the rejection of everything that flows is related to the fact that flows do not have a specific object. They not only stand for the masses of soldiers that need to be defeated, but also for political, literary, and intellectual currents, and by extension also for streams of hidden, internal desires which lack specific objects.
} 
Back then, William Hague of the Conservative Party used words such as 'flood' and 'swamped' in the context of discussions around asylum seekers, words which "create associations between asylum and the loss of control and hence work by mobilizing fear, or the anxiety of being overwhelmed by the actual or potential proximity of others" (Ahmed 2004, 46). The point is that these words or 'signs' were repeated in similar or (supposedly) related discussions during subsequent years, and "became 'stuck' or 'attached' to the 'asylum seekers' speech, [...] also through the repetition with a difference, of some sticky words and language" (Ahmed 2004, 46, emphasis in original).

With her emphasis on repetition, Ahmed is here pointing to Judith Butler's work on the stabilization of norms, through "reiterative acting" which becomes "a process of materialization that stabilizes over time to produce the effect of boundary, fixity, and surface we call matter" (Butler 1993, 9). In the same way as norms become stabilized, a certain 'stickiness' is achieved when the 'flood' and related figurative signs are used repetitively in slightly different contexts. In an affective economy, feelings do not reside in subjects or objects, but are produced as effects of circulation. In these circulations, feelings may stick to some objects and slide over others (Ahmed 2004, 8).

The fact that the flood metaphor and its variations re-appear (e.g. 'swamped', 'drowning in') again in 2016, when it re-appears in the filtered comment of the sample on hatr.org, shows how it is circulating: between different countries (in this example, Germany and the UK) and between different discourses (on 'bogus' asylum seekers in Ahmed's example, the refugee crisis on hatr.org). It also shows how the stickiness of the flood metaphor applies to diverse groups of people (e.g. asylum seekers, immigrants, refugees) with different situations, motivations, and goals who only have in common the fact that they are considered to be 'the other'. 


\section{Other Stickers}

While the flood metaphor is here circulating in the context of questions of race and ethnicity, other 'sticky' signs are evoked in similar ways in the present sample; namely, in relation to gender and sexuality. Several of the hate commentators move onto highly intimate levels and relate to embodiment, genitalia, sexuality or femininity to create and underline an unlikeness. In the context of race, Ahmed argues that:

Hate involves the surfacing of bodies through how we encounter others in intimate and public spaces. The politics of racial hatred involves attributing racial others with meaning, a process we can describe as 'the making of unlikeness'. Hatred is a negative attachment to an other that one wishes to expel, an attachment that is sustained through the expulsion of the other from bodily and social proximity. $(2004,55)$

Put differently, in this way, words of hate passionately seek to expel the other, to cut one's attachment to it, by creating the greatest possible difference from oneself or from what one considers to be the norm. The result in my hatr.org sample is that what 'sticks' to the bloggers being attacked here is the violent sexualization of their bodies (in the case of sexist slurs), a direct marginalization and expulsion by naming them as part of a fringe group or commanding them to leave the country, or animalization and fat-shaming (e.g. by comparing the blogger to a whale).

It is interesting that, even though the context of these hate comments is missing, they work with sticky signs that are nevertheless recognizable, because they use signs which have been circulated, repeated and are parts of pre-existing emotionally charged discourses. Through repetition and circulation, these figures of hate have accumulated their affective value, "precisely insofar as they do not have a fixed referent" (Ahmed 2004, 47).

In the present sample, tropes of female sexuality as excessive or obscene, and in need of being punished or destroyed, are employed. So 
is the trope of the ugly feminist or of women as being closer to the animal kingdom than to humans, which are sticky signs with long histories of false dichotomies and hierarchizations, circulated again. In other words, what is evoked here is what Braidotti called "woman, the sexual other of man; the ethnic or native other of the Eurocentric subject; and the natural or earth other of technoculture" (Braidotti 2002, 166). The goal is for the attacked bloggers and their positions to be pinned down outside of the norm, defined as 'different', in the sense that different here means 'worse' than an assumed norm of the 'us'.

Having assessed how these economies of hate work and what their tools are - attaching signs to particular bodies and not to others; creating unlikeness to the assumed self or norm and the repetition of these signs with no or small differences -in the following I will analyze what hatr.org is doing to disrupt or confuse these economies.

\subsection{Displacing Hate}

All of this use of figurative signs that aim to align with some bodies and expel others, the making of unlikeness, and the attempt to turn bodies into objects, are part of affective economies, in this case the affective economy of hate. "Hate is economic, it circulates between signifiers in relationships of difference and displacement", as Ahmed explains (2004, 44). Indeed, leaning on Marx's critique of the logic of capital, she understands that:

[a]ffect does not reside in an object or sign, but is an effect of the circulation between objects and signs (= the accumulation of affective value). Signs increase in affective value as an effect of the movement between signs: the more signs circulate, the more affective they become. (ibid.)

In this vein, it can be argued that the strategy of hatr.org is to disrupt and twist this affective economy, its circulatory movement of hate 
speech and, consequently, the accumulation of affective value. This is happening on several levels.

The first interruptive cut is the concrete, immediate result that the viscerality of the experience is removed for the individual subject. When the hateful comments are displaced, the affected bodies are not moved along at the same time. The author of the blog entry being attacked will not read the insult and will not be affected by it. Consequently, the hate message is no longer connected to the real, which is the writing, body. The materialized rhetoric and affective power of hate are grasping at nothing.

For the individual who is shielded from the hateful message by hatr, the 'stickiness' of figurative language no longer applies. Instead, a forced 'sliding off' is taking place, and with it the pressure for the addressee to embody a particular identity is dispersed. The hateful comment - which becomes an emotional object - is re-directed, so the circulation is disturbed and guided into an elsewhere. In this way, the hate comment becomes less affective because it does not hit the desired target.

\section{Relaying Expulsion}

Instead, the act of displacement of hate messages to hatr.org creates a decontextualization. As Ahmed has argued, hate is "a negative attachment to an other that one wishes to expel" $(2004,55)$. But, through hatr.org, this expulsion is not taking place (the object of hate cannot be expelled because it does not receive the message). Instead, the hater is expelled to another place.

This wish to expel the other mirrors what Judith Butler wrote about the context of hate speech, where the expulsion becomes a way to put the addressee out of control and into a different location. She says, and I quote at length:

The speech situation is thus not a simple sort of context, one that might be defined easily by spatial and temporal 
boundaries. To be injured by speech is to suffer a loss of context, that is, not to know where you are. Indeed, it may be that what is unanticipated about the injurious speech act is what constitutes its injury, the sense of putting its addressee out of control. The capacity to circumscribe the situation of the speech act is jeopardized at the moment of injurious address. To be addressed injuriously is not only to be open to an unknown future, but not to know the time and place of injury, and to suffer the disorientation of one's situation as the effect of such speech. Exposed at the moment of such a shattering is precisely the volatility of one's 'place' within the community of speakers; one can be 'put in one's place' by such speech, but such a place may be no place. (Butler 1997, 4)

The addressee finds herself without context or control, her body is in a place of no place and spatial and temporal boundaries become blurred. The hate is aiming to expel the addressee from their context, to render them out of control, into disorientation. In the concrete example of hate speech on hatr.org, the troll or hate speaker seeks to make one's place volatile - sometimes literally, when the hater tells the blogger to leave the country or questions the blogger's right to exist in their society.

When slurs and swearwords are used, the addressee is additionally expelled from bodily or social proximity, an unlikeness is created and underlined (for example, when terms such as 'whore' are used, I understand this as way to construct an unlikeliness based on a gendered category of what is usually considered to be a social outcast).

What happens when the hate speech about which Butler is writing is taken online, where the sender of the hate speech usually remains anonymous? I suggest that in this case the experience of disorientation and loss of control is amplified; out of a perceived nowhere, the addressee suddenly finds herself in a volatile position that is even more dislocated because the sender of the message usually cannot be located and remains invisible. 
I would like to argue that what hatr.org is doing to the comments/commentators reflects aspects of what the haters do/want to do to the bloggers. The displacement of the hate comment equates to a loss of context; it renders not the addressee, but the sender, out of control. The haters have to ask themselves: Where did my comment go? Why is it not showing? ${ }^{63}$ It can be assumed that there is a moment of disorientation, which, albeit certainly not as drastic as the violence which the blogger who is faced with hate mail has to endure, is kind of a 'place of no place'. As a result, the hater is left with a double-nothing: The body of the hate object cannot be grasped and one's own position/place, which is a positon of assumed superiority, becomes shaky. In this context, it is interesting that Ahmed sees hate as a two-way street, indeed as a form of intimacy:

To consider hatred as a form of intimacy is to show how hatred is ambivalent; it is an investment in an object (of hate) whereby the object becomes part of the life of the subject even though (or perhaps because) its threat is perceived as coming from outside. Hate then cannot be opposed to love. In other words, the subject becomes attached to the other through hatred, as an attachment that returns the subject to itself. (Ahmed 2004, 50)

Through hatr.org's strategy of displacement and decontextualization, the hatred cannot result in the negative attachment that the hater sought. Therefore, following Ahmed's understanding of hate as a form of intimacy, haters re-published on hatr.org have lost their investment and have missed the opportunity to make the object part of their lives.

\footnotetext{
${ }^{63}$ Although many commentators probably assume the blogger or the editorial board have simply deleted the comment, which would mean that the hateful message has indeed affected the desired goal (Being read and getting a response). However, this strategy of displacing the text seems to be a particularly apt strategy for trolling. In the original meaning of the term, the online troll seeks to steer the discussion onto a different path, to cause confusion, outrage, or a change of topic. That is, it can also be seen as a strategy of confining someone to 'a place of no place'.
} 


\section{Reacting to Hate}

I understand hatr.org's strategies to counteract hate speech in the following ways: Firstly, hatr aims for a concrete disruption of the connection between individual and collective bodies and the content of the hate message in question. To put it differently, hatr uses cutting and pasting from one forum to another, where the body is not 'dragged along'. This step protects the blogger's integrity and contributes to a harassment-free communication culture.

Secondly, the decontextualization, that is, the way in which the hate comment is taken to 'a place which is no place', and which also interrupts the intimate attachment that the hater has to the object of hate, might leave the hater in a mild state of puzzlement and dissatisfaction. In a way, this aspect might be the one that is most likely to be seen as a 'coming back', a penalizing act, which resembles censorship - an aspect to which I will return in the next paragraph. For now, and following Butler, it can be said that this means that, by displacing the message, hatr.org does exactly the same to the sender of the hate message as its intention was to do to the blogger: robbing it of context and rendering it out of control.

\subsection{Generating a Digital Testimony}

The anti-hate mail strategies analyzed above are of a rather reactive, or, if you like, defensive nature. They aim to react to particular threats and insults. I have argued that doing digital media differently should not solely be understood as reactive, but also as generative, in that it aims to enable counter-subjectivities and offer alternatives to plain good/bad dualisms and instead provide another kind of 'difference'.

So far, however, the processes described still resemble aspects of censorship, a fact which opens up a completely different set of issues. Judith Butler's critique of censorship in Excitable Speech: A politics of the performative (1997), for example, criticizes the role of the state and its implicit, Foucauldian power over acceptable discourse and argues 
that censorship might reinforce the very same language it seeks to forbid.

This is not the case in my example, but it mirrors the caution of some feminist activists about relying on legal measures and who are consequently searching for other alternatives, of which hatr.org is one. Secondly, Butler's complication of censorship is based on doubts about its efficiency: She argues that hate speech always depends on an embodied context, and that it is therefore not possible to define particular words or phrases as always being hateful. The problem is that it is difficult to find a system to identify hate speech, because hate signifiers can appear in so many different ways depending on an individual's embodied subjectivity (think, for example, of the Slutwalk organizers' attempt to mobilize a slur in a different context).

Even though Ahmed agrees with the argument that hate cannot reside in particular signs or bodies $(1997,59)$, she argues that Butler did not sufficiently take into account the affectivity of hate speech, in the sense that affects are not determined in themselves but produce particular affective responses by drawing on "histories that have stayed open" and in which "such names assign the other with meaning in an economy of difference" (e.g. racist discourses, ibid.). This is why particular words are repeated in affective economies and how they are affectively charged.

I agree with Ahmed here and argue that hatr.org is an excellent example of this. Even though the embodied context (in the sense of the body of the recipient of the hate message) is missing, the words used in the present sample refer to particular 'histories left open' and they are affective in and of themselves to the reader (hence the trigger warning).

In this way, I suggest, hatr.org is offering a third way, a different way of handling online harassment: the messages are neither deleted in the name of censorship nor left untouched in the name of 'freedom of speech', but redirected. They become "open to an unknown future" themselves (Butler 1997, 4) when displayed on hatr.org. On the one hand, this means that 'the tables are turned': the comments become vulnerable by being left open to scrutiny by users who visit the anti- 
trolling platform. Out of context, the messages appear even more absurd, unsubstantiated, and furious. Even though the point of reference is missing (in the sense that the original blog post is not displayed), they seem disproportionate, and quite literally 'out of place'.

On the other hand, the figurative, sticky signs that were circulating before are now stuck in one place, and recognizable to the reader as a repetitive pattern. The system is exposed. In this way, hatr.org is building a digital testimony, which is affective to readers. A documentation of what the climate of current online discussions can look like is taking place, and this might be a useful tool for starting a discussion on online harassment, cyberbullying, and hate speech online. Instead of deleting the messages, which would be a simple form of censorship, they remain visible within the critical framework of hatr.org.

The creation of a digital testimony of something that is as difficult to grasp as online harassment is thus the first result that I would describe as generative: it generates by becoming affective to the reader, who may see that the comments could just as easily be directed towards themselves. What I mean is that, even though the displaced messages are no longer connected to a particular body, they are still affective to the reader of hatr.org, who becomes a witness thereof.

Without the original blog post displayed, the reader knows neither where the message came from nor who sent it to whom or in what context this happened. One does not know what the comment has been written in response to, so the original blog post that was attacked could have been something written by oneself or something one might have thought or supported. The database does not allow to doubt the embodied nature of hate comments, because the comments are still affective and now they affect the hatr.org reader in their intensity.

\section{Monetizing the Hate}

The other generative factor is that hatr.org is literally generating money. As I explained in the introduction to this chapter, hate com- 
ments are indirectly turned into revenue by putting sponsored advertisements onto the page. ${ }^{64}$ Tongue in cheek, hatr.org argues that they are trying to 'cold-bloodedly monetize the trolling'. ${ }^{65}$ In a kind of ironic twist, an economy of hate is here turned into an economy of cash.

Even though it would be difficult or even impossible to counterbalance injuries received through hate speech with plain money or to 'measure' their affectivity, I argue that trying to monetize the hate is an important and thought-provoking gesture. It makes hate more graspable for bystanders, and symbolically confers 'value' to a supposedly disembodied, or solely virtual phenomenon - hate online. In this sense, hatr.org is building a counter narrative to digital dualist arguments that what happens online does not matter, or matters less. The materialization of hate into cash is mimicking or mocking the material dimension of hate.

In this context, it is interesting to consider globalization scholar Saskia Sassen's discussion of digital-material imbrications and the embeddedness of digital technologies in societal structures and power dynamics (2002). She argues that dematerialization, which is often seen as a feature of new technologies, is actually only one side of the coin, since this dematerialization depends on reintroducing non-digital variables. Dematerialized money transfers, for instance, require "enormous amounts of material, not to mention human talent" $(2002,369)$. To sum up, "much of what is liquefied and circulates in digital networks and is marked by hypermobility, remains physical in some of its components" (ibid.). In a similar fashion to Sassen's reminder of the human aspect and material dimension behind 'de-materialized' money transfers, hatr.org's 'monetizing-the-hate' strategy hints at the fact that hate is also not dematerialized only because it happens online.

64 In June 2016, a web shop for 'computer geek' supplies posted several banners. It is also possible to make donations via the donation service

flattr.com, which seeks to support open and free web content (last accessed 7/06/2016).

65 http://hatr.org/about, last accessed 15/10/2016. This translation from German to English is mine. 


\subsection{Discussion: Hatr's Strategies as Feminist Activist Tool}

Hatr is an initiative that neither abandons the addressee of the hate mail, nor solely censors or deletes the messages. Instead, something different is created: firstly, an archive that can be seen as a testimony to the dimensions and intensities of hate speech online. I consider this very important since so much of what is happening to people online lies in a legal grey zone, often silenced, ignored, or downplayed. It is something that often cannot be proved as a form of violence because it does not cause bruises or wounds (Ahmed 2004, 58).

And secondly, it generates plain old cash and thereby turns something 'bad' (the hate) into something 'good' (in the sense that money is used for a good cause). The money-making aspect must not only be seen from an economic perspective; in addition, I would argue that it is relevant because it simply positions something graspable in opposition to the hateful messages, and almost turns their intention into its opposite (for example, when money earned through transphobic messages indirectly generates income for an organization that supports trans causes).

In this way, the indirect message that their hate is being redirected to another cause is sent to the haters, very probably a cause that is detrimental to what the hater had in mind with their insult - even though most of them will probably never know what happened to their comment. 66

I have discussed hatr.org and its strategies in this first empirical chapter because online harassment is a particular digital inimicality that makes life for women and other others more difficult, and I see a need to find feminist political strategies and tools to counteract it. Currently, strategies for how to handle online harassment are still in their

${ }^{66}$ It has been reported, though, that some persistent haters include messages along the lines of 'Why don't you put this on hatr?' to the hateful messages, well aware that their comment might end up there (Reinsberg 2013). 
infancy; this is why I have evaluated strategies to do digital media differently in the context of inimical online communication cultures.

The hatr.org project reminds the reader of how words wound and how they can put the addressee affectively into a place of no place (Butler 1997, 4). But hatr.org goes further: it is a unique attempt to make the insults and the aggressors more tangible. Here, words can be grasped, displaced, and in an ironic twist even be monetized. In this way, they become detached from the bodies at which they were aiming, and become dislocated instead, as though what they were intending to do to the addressee is suddenly being done to themselves. At the same time, I have argued, hatr.org is a strategy that sidelines current, common strategies of deleting and censoring nasty online comments and instead offers ways to do digital media differently: by creating a digital testimony and underlining the affective, material dimensions of hate. Building a digital testimony is important because cyberbullying does not cause visible bruises:

Hate has effects on the bodies of those who are made into its objects; such bodies are affected by the hate that is directed towards them by others. [...] The effect of hate crime is affect, and an affect which is visceral and bodily, [...]. [T] he body of the victim is read as testimony, as a means by which the truth of hate crime is established in law. (Ahmed 2004, 58)

The trick is that bodies become testimonies but the actual individual body is saved, spared. For this reason, I think that hatr.org's attempt to react to online hate and to generate something from it builds an interesting answer to the question of feminist responses to inimical digital communication cultures. It aligns well with feminist politics for several reasons: Hatr.org is sidestepping the difficult question of censorship as posed by Butler by finding an alternative that does not lose the affectivity of particular signs in affective economies of hate. By creating a digital, affective and embodied testimony, hatr.org furthermore acknowledges that the body does not end at the keyboard, by making the hate speak more visible, graspable, and affective to the reader. This 
strategy differs from others that have been popularized in recent years; for example, the moderating of comment sections by the writer of the article/blog entry, ${ }^{67}$ because it does not allow the hate messages to affect the writer's body while still becoming part of a digital testimony.

Another reason why hatr's strategy is an interesting answer to the question of feminist responses to inimical digital communication cultures is that it is connecting to earlier, classic forms of feminist activism, such as awareness raising or consciousness raising. After all, cataloguing sexism remains a crucial form of feminist activism (Ahmed 2014). 68

There are of course also limitations to hatr.org's approach. As I see it, a major factor for criticism could be the fact that the problem is simply 'outsourced' to a different person, who has to do the work of filtering the comments. In this sense, even though the filtering person might be able to take the comments as less personal than the original blogger would have (they might 'slide off' of them), it is nevertheless still a deferral of the problem. There is still a person interposed between hate and displacement, who does not get a 'trigger warning', and there is still a person 'out there' who intends to hurt another person with violent words. The circulating signs of hate, even when they are decontextualized and redirected, are still 'sticky' and harmful.

Additionally, there is still an investment of time and labor power that this voluntary work requires and which surely cannot be covered by money generated through advertisements (which is in any case promised to charitable causes). This line of thought could be a question for further research: What are good strategies to handle these huge

\footnotetext{
${ }^{67} \mathrm{~A}$ common strategy today is to delete the offensive message and post an automated message that reminds readers of the forum's communication guidelines.

68 Elsewhere, Ahmed talks about forms of feminist online activism like the Everyday Sexism Project (http://everydaysexism.com/, last accessed 08/o6/2016) as a contemporary example of the necessity to catalogue sexism as a collective project.
} 
amounts of digital hate and other kinds of offensive materials? ${ }^{69}$ I see hatr.org's strategy as a first attempt from a feminist activist perspective to productively answer this question, which I believe is a question that will stick in the years to come.

In my next empirical chapter, I enter a different world of engaging with digital inimicalities, which are more sublime.

69 Parallels can perhaps be drawn with recent news reportage on the working conditions of 'content management workers' in Manila. In order to keep social media sites free from pornographic material, extreme depictions of violence (e.g. car accidents or beheadings), sodomy or other crass material, predominantly female workers with 'Christian values' are hired to 'clean up' social media feeds for Western mainstream compatibility (Riesewieck 2016). This recalls Donna Haraway's critique of what she called 'homework economy' (Haraway 1991, 166), that is, the feminization of labor in the new economy, and brings it into the arena of digital communication culture. 

GETTING INTIMATE WITH CODE CODING AS AN ON/OFF LOVE STORY 



\section{Getting Intimate with Code: Coding as an On/Off Love Story}

This second empirical chapter investigates initiatives that try to make women* more familiar with coding. They are trying to reframe the image of coding, which historically has been saturated with ideas of detachment, solidity, and masculinity, as a rather social and passionate activity instead. After tracing how these cultural parameters came into being historically, I analyze the use of intimate notions in the coding initiatives' promotional material and the implementation thereof within an actual workshop that I took part in. Based on my findings, I furthermore discuss how the use of intimacy here is related to what Michael Hardt has called 'affective labor' (1999).

\subsection{CodeGirl}

The 2015 documentary movie CodeGirl by filmmaker Lesley Chilcott follows several groups of high-school girls who have entered the "Technovation Challenge": an international competition in which teenage girls from all over the world develop apps to tackle particular societal problems in their respective communities. ${ }^{70}$ The movie opens with a visit to the 2014 winners of the competition: The team "Health in a Drop" from Moldova convinced the jury with their app Apa Pura. The problem this app addresses is that in the girls' village, Ştefăneşti, the water quality from public wells is poor and it can cause illnesses such as Hepatitis A. Nevertheless, many locals use the wells because bottled

\footnotetext{
${ }^{70}$ The organizer of the event, Technovation, is an NGO that describes itself as a "global technology entrepreneurship program for girls" (http://www.technovationchallenge.org $/$ ?page $=5$ ?page $=1$, last accessed 13/08/2016). The Technovation challenge has been running since 2010 and altogether over 5000 girls from 60 countries have signed up for it. Of these, a few teams are pre-selected in national competitions to take part in the threemonth program that prepares them for the final competition.
} 
water is too expensive. Apa Puro, the girls' app, helps to locate wells (via geotagging) and lists water quality with a rating system.

The footage during these first few minutes sets the mood for the entire documentary: It shows inspired young women who have learned to utilize novel technologies and entrepreneurial strategies and are enthusiastic about their success. The "Health in a Drop" team is shown as they draw a simple bucket out of the well, commenting to the camera team: "This is our water. If you want, you can try but I don't. (laughter) I don't want. It has a bad taste and smell". The fact that they are located in a financially struggling Moldavian city with a rural feel amplifies the contrast between novel technologies and their industries and the girls' lived experiences, where finding clean drinking water is a daily hassle. As though to amplify the contrast further, the girls can be seen smiling brightly during the winner's ceremony in San Francisco, with flower chains around their necks and 10 ooo US Dollars of start-up capital for their enterprise in their pockets, in one of the next scenes.

During the remainder of the movie, several teams from such diverse countries as Nigeria, Mexico, the USA, and France are followed as they develop business plans, learn basic coding to create a prototype of their app, make a pitch video to promote their idea to a jury, or brush up their English for presentation day, all with the help of assigned mentors.

\section{Emotionality as Thematic Concept}

The mood set in CodeGirl is one of affective empathy and sympathy for the contestants, who occupy a kind of underdog position from which, against all the odds, they have been given the chance to become winners of an international app competition. The documentary is full of images of intense concentration, teamwork and friendship, hope and disappointment, and makes the viewer cheer for the young women. As is common in human-interest documentaries, personal stories, intimate interactions, and close-up shots of the protagonists define the movie. 
In this way, the viewer becomes emotionally engaged. Its affectivity is achieved by, for example, the girls being shown in their everyday surroundings, such as their homes or classrooms. They are shown sharing emotions like nervousness, tension, joy, or disappointment and appear to be close to each other. This is also underlined when the voiceover in the intro states that "For most of them, it is not about winning", a statement which proposes a higher and purer goal like empowerment, self-development, or everlasting friendship. It can thus be said that emotionality is a thematic concept within the documentary. The viewer feels with the protagonists and is touched by their emotional investment.

The intended purpose of director Chilcot is to promote the challenge and to encourage more girls to become interested in technology, coding and its business. ${ }^{71}$ CodeGirl wants to change the status quo: "It's a $\$ 77$ billion industry that's 80 percent male. One worldwide tech contest tries to narrow the gender gap." ${ }^{2}$ The gender-gap message is underlined throughout the film; for example, when one of the competing girls is quoted as saying: "Part of being a female programmer is that you are feeling really lonely" or when one of the mentors describes how technology is constructed as "not for women" in her home country of Mexico. The image of gender and coding that is provided here is thus one of difficulty and tension. For girls, according to the underlying message, it is tough to prevail in technological domains.

I would argue that CodeGirl is a movie of its time because it captures a particular cultural phenomenon or societal trend: the idea that STEM fields in general and computer subjects in particular, whether scientific or practical, are inimical fields for girls. The imagined solution to this problem is what Monica Obreja has called the "critical mass" approach; the idea that if a certain number of women were to

\footnotetext{
${ }^{71}$ As she also says in an interview, http://www.themarysue.com/interviewCodeGirl-director-lesley-chilcott/, last accessed 20/07/2016.

${ }^{72}$ Promotional synopsis of the movie on the streaming platform Netflix, last accessed 01/08/2016.
} 
enter a field, the culture would change $(2012,177)$. The proposed solution is to offer girls extra coaching and tutoring that is supposedly more suitable to some imagined set of feminine needs and values.

The broader social trend that filmmaker Chilcot is portraying thus consists of an increasing awareness of gender gaps in tech industries and, to a lesser degree, of a need for more intersectional initiatives to diversify tech businesses and cultures. This is visible in documentaries, 73 popular culture or state-subsidized programs. For example, free or affordable workshops and courses, which are mainly provided by non-profit organizations, have proliferated in recent years, ranging from grassroots DIY initiatives to Google's recent 50-million-dollar campaign, Made $w /$ code.

In line with my main research questions, the overall purpose of this chapter is to analyze such coding initiatives for women* and their strategies as analytical-practical tools for a feminist toolbox to counteract digital inimicalities. As a first step, I engage with the promises made by organizations that offer free or affordable coding trainings, such as Girl develop it, Railsbridge, Ladies Learning Code, The Women's Coding Collective, Railsgirls, Codebar, or Blackgirlscode, in their mission statements or promotional material. The point is that, here as well, similarly to the movie CodeGirl, emotional and social concepts are stressed, an approach which creates particular expectations of how the courses/workshops will be different from more mainstream ones and how being a girl or a woman plays into this.

In other words, the task is to evaluate their approach of (re-) framing coding as a collective and somehow emotionally charged experience. Is this a potentially valuable strategy for confronting gender and diversity gaps in coding and disrupting gridlocked stereotypes about women's limited compatibility with coding (or technology in general)?

I analyze how these contemporary women*-centered initiatives 'do digital media differently' by discussing whether and how notions

73 Somewhat similar to CodeGirl, the 2015 documentary $C$ q de - Debugging the Gender Gap by Robin Reynolds (2015) tackles the issue of a gender gap in coding and its industries from a wider angle than CodeGirl, for example by providing a historical background to its emergence. 
such as intimacy, femininity, masculinity, technology, and digitality are adopted, changed, and developed therein. More specifically, I am interested in how they are changing within a context of digitalization and its contemporary embedding in a neoliberal market logic (e.g. Web 2.0).

Following my intention of developing the notion of digital intimacy in theoretical terms, I approach the phenomenon from different angles in order to conjoin them into a cultural analysis. I see this as an important step in understanding how gender constructions are the product of different social technologies or, as Teresa de Lauretis, a foundational figure of feminist cultural approaches to gender and technology (1987) puts it, a product of

\begin{abstract}
movement between the (represented) discursive space of the positions made available by hegemonic discourses and the space-off, the elsewhere, of those discourses: those other spaces both discursive and social that exist, since feminist practices have (re)constructed them, in the margins [...] of hegemonic discourses and in the interstices of institutions, in counterpractices and new forms of community (de Lauretis 1987, 26).
\end{abstract}

Firstly, as one such historical techno-social apparatus, I give a short overview of the history of women and coding, and more specifically how this history has been influenced by notions of intimacy. Indeed, for a long time this history has languished in an 'elsewhere' and has only been (re-)discovered in recent years. I trace how it can be seen as a 'back-and-forth' movement between "hegemonic discourses and the space-off" (ibid.), and how normative parameters of femininity and masculinity vis-à-vis coding have influenced, guarded and changed affective and intimate relations between bodies within socio-technical networks.

Secondly, moving on from this historical background, I turn to current-day women and coding initiatives. I introduce some of the bigger, better-known initiatives that I came across and which have fre- 
quently been discussed in media reportage or the blogosphere. I discuss them primarily by focusing on their self-presentation or mission statements on their homepages. What is it that they are striving for and what do they promise to participants?

Central to this discussion is an analysis of my own experience of taking part in such a women*-centered coding workshop, which also promised a different and more social coding experience. Invoking Haraway's "ethnographic attitude" (1997), I draw on fieldnotes from joining this workshop myself as participant and observer.

One of the main findings from my fieldnotes is that intimacy in digital times has to be seen in the context of the entrepreneurial self (Brown 2003; Scharff 2014)and affective labor (Hardt 1999). In the final part of this chapter, I therefore discuss a certain field of tension between (cyber-)feminist ideals and the neoliberal contexts in which these women*-centered groups are embedded.

By shedding light on this contemporary social phenomenon of manifold initiatives to bring girls and women into coding from multiple perspectives - historically, with an ethnographic attitude, and critically - I aim to deliver a snapshot of the relationship between gender and coding at this current moment. I zoom in on particular aspects of the phenomenon that illustrate the changing nature or unsteadiness of this relationship in a context in which digital technologies have become an intimate presence that increasingly influences who we are and what we do.

\subsection{An On/Off Love Story with Coding}

What the coding courses, the movie CodeGirl, and other STEM-focused initiatives targeting women suggest is that we are at a point in time when there is a cultural agreement that it is necessary to get women into tech and that novel strategies are needed in order to do so. How did the idea that women and coding do not go together smoothly come into being in the first place? My task here is not to give an all-encom- 
passing overview but to focus on the role played by intimacy in the historical and cultural construction of the women and coding relationship (for a broader exploration of the history of women and coding, see Misa 2010). How did this love story begin and how did it develop?

As a starting point, Ada Lovelace's work with and impact on Charles Babbage's Difference Engine and Analytical Engine is often mentioned. She is commonly credited with publishing the first program for the Analytical Engine, her Notes, which can be described as the first form of computational algorithm. Back then, the Victorian mathematician called this early coding, which later earned her the title 'first programmer in the world', a "poetical science" (Isaacson 2014, 162), as though to blur the boundaries between hard sciences and fine arts. Symptomatically, she was the daughter of a famous poet (Lord Byron), in whose absence she was raised, whilst her mother, Anne Isabella Milbanke, as a kind of 'antidote' to the father's poetic legacy and bohemian lifestyle, emphasized an education that was marked by discipline, hard study, and a focus on mathematics. However, at this initial point in the history of modern computing, it seems that passion and the poetic played a role in what coding might become.

After falling into oblivion for a century or so, in more recent years Lovelace has become a kind of poster girl for women's pioneering role in computer science.74 The interest in her parallels a general increase in attention to the promotion of women in STEM (Carlucci Aiello 2016, 58 ). This might not only be due to her exceptional position as woman and pioneer, but also because she symbolizes a different approach to computing, due to the fact that early computing functioned in ways so

\footnotetext{
74 In tandem with the renewed appreciation of Lovelace's contribution, there have also been doubts raised about her actual degree of involvement and relevance. But Thomas J. Misa, historian of the Charles Babbage Institute, Minnesota, points out that the collaboration between Babbage and Lovelace was marked by passion (and sometimes animosity), that they were, even though not a couple, "intellectually intimate" (Misa 2015, 12), and that their contributions cannot be seen as a "zero-sum game" (Misa 2015, 14), in the sense that one's contribution does not detract from the other's. His discussion points to the fact that it was and is difficult for women to receive recognition in this area, which indicates an 'off'-moment.
} 
different from how digitality is perceived today. The Analytical Engine managed to accept data and programs as input by reading punched data and programs on cards similar to those used in mechanizing textile looms (ibid.). Lovelace is said to have been inspired by the technologies of the loom and to have developed the historic insight that Babbage's machine could handle many kinds of data, which could be notated in logical symbols, like music, graphics, or words.

In this sense, Lovelace and Babbage's working conditions were very mechanical, material, and graspable, which contrasts with a commonplace present-day perception of the workings of digitality as abstract, immaterial, and opaque. Therefore, it might be the case that the appreciation of Lovelace as an idol is also related to a longing to make software and code more tactile. There is support for this claim in, for example, her popularity as an icon in steampunk literature and fan art. Together with Babbage, she has become the heroine of steampunk-inspired comics 75 and countless internet memes. Many branches of the steampunk subculture engage with the material, affective, and tactile dimensions of Victorian (steam-powered) technologies and in a kind of retro-futurism blend them with today's digital technologies and media (Sundén 2014). Ada Lovelace not only represents being a pioneering woman in coding, she also stands for a time when computing meant mechanical and graspable interactions with machines and she considered it to be poetic rather than prosaic. In other words, I want to suggest that her approach is an affective one, perhaps more obviously affective than a modern conception of digitality as supposedly "vacated of passionate connectivity" (Sundén 2015a, 135).

While it has been said that Lovelace and Babbage's work led to the dawn of the information age roughly a hundred years ahead of schedule, the beginning of modern computing entails another story in which women were the 'firsts'. From the 1950s on, women executed profes-

75 See, for example, Sydney Padua's affectionate fanfiction comic/textbook The thrilling adventures of Lovelace and Babbage (2015), in which Lovelace does not die from illness at the age of 36 (as she did in reality) but instead has futuristic adventures in parallel universes. 
sional programming and it originally had the reputation of being a female-friendly vocation (cf. Ensmenger 2010). The ENIAC girls ${ }^{76}$ were celebrated as the world's first programmers and Cosmopolitan magazine rejoiced: "Women are 'naturals' at computer programming" (Mandel 1967, 52). 77 Coding was understood as a fun, social, and collegial work experience in this (Isaacson 2014) and the following decades (Abbate 2010). In other words, this could be said to be another 'on' moment in an on-off relationship.

However, it did not stay that way. Nathan Ensmenger, a historian researching gender and computing, traced how an increasingly male subculture of computer hacking from the 1960 s onwards became "reinforced and institutionalized" (2010, 77), mainly through the hiring practices of the industry. Job descriptions started to emphasize a need for mathematical skills and the ability to work sealed off from one's surroundings for long periods of time. These qualities were considered to be masculine and they privileged typical male educational experiences, but they were in fact not really requirements for successful programming $(2010,77)$ nor in anyway inherently masculine.

But privileging male educational experiences was only one side of the coin:

Even more obviously gendered were the personality profiles that reinforced the ideal of the 'detached' (read male) programmer. It is almost certainly the case that these profiles represented, at best, deeply flawed scientific methodology. But they almost equally certainly created a genderbiased feedback cycle that ultimately selected for programmers with stereotypically masculine characteristics. The

\footnotetext{
${ }^{76}$ Electronic Numerical Integrator and Computer, the world's first generalpurpose computer with a weight of 27 tons.

77 As though in a blast from the past, the coding for women's organization Code First: Girls teamed up with Twitter and fashion magazine Elle in spring 2016 to host a workshop: "Code your Way to a Career in Fashion" [http://www.elleuk.com/life-and-culture/news/a29402/code-your-way-toa-career-in-fashion-with-elle-twitter-code-first-girls/last accessed 15/07/2015].
} 
primary selection mechanism used by the industry selected for antisocial, mathematically inclined males, and therefore antisocial, mathematically inclined males were overrepresented in the programmer population; this in turn reinforced the popular perception that programmers ought to be antisocial and mathematically inclined (and therefore male), and so on ad infinitum. (Ensmenger 2010, 78-79)

Even though, as Ensmenger argues, this feedback loop was not so much deliberate as "convenient - a combination of laziness, ambiguity, and traditional male privilege" (2010, 79), during the 1980s this stereotype of the male computer geek or hacker became more widespread, "paralleling - and perhaps contributing to - the decline in women's participation" (Abbate 2010, 215).

This means that an abstract notion of 'detachment' became a cultural parameter for success in a rapidly growing technological domain. Detachment is seen as an assumed capacity to be unaffected by one's surroundings, such as the idea that one can detach oneself from everything in the vicinity and lose oneself within the assumed immateriality of the digital.

This parameter of detachment builds an opposition to the intimate in the sense that intimacy relies on mobile, affective attachments. In the context of coding, it became a false ideal, featuring, for example, portrayals of computer professionals working in solitude or in hostile, overly competitive environments, and as being disconnected from 'real-life' issues (Abbate 2010, 214). It is also a false ideal because it is based on a variation of Cartesian dualism, which is the idea that the mind can be seen as distinct from the body and includes the hierarchization of the former over the latter, a dualism that has traditionally worked to the disadvantage of women, who were usually related to the realm of the body. Subsequently, this idea of the detached programmer has often been implemented in media representations, job descriptions, recruiting assessments, educational practices, and workplace cultures (Abbate 2010, 15). 
Once this feedback cycle of a "highly masculine subculture" had been set in place, a cultural process of estrangement from the machine took place, sometimes construed along the lines of sexuality. Sherry Turkle investigated the culture of student computer hackers at the Massachusetts Institute of Technology in the 1980s, and the results were published in her influential book The Second Self: Computers and the Human Spirit (1984 [2005]). At that time, she argues, a moral discourse of relationships with the machines replacing or substituting for social contacts was set into place ([1984] 2005, 194). The worry was that this would lead young men (since it was usually young men) to become too detached from their surroundings, that they would become unproductive, lose interest in other activities, including sexual ones, and instead only "love the machine for itself" ([1984] 2005, 183ff.).

In a later piece of research with some of the few female students who were studying computing (1988), she diagnosed a rejection of this kind of intimate relationship with the machine. The young women were asking themselves if they belonged in a world that "takes the machine as a partner in an intimate relationship" (Turkle 1988, 34). They were shying away from a model of intimacy that is so obviously object-oriented; it did not match what were perceived as feminine values (e.g. being social, caring for others).

From a 2016 perspective, her findings sound almost peculiar considering the intimate presence of everyday technologies in today's world, and the extent to which 'subject-oriented' intimacy is arranged by them (be it in the form of a smartphone tracking personal health data, online dating, or our constant physical closeness to mobile devices).

I find it remarkable how intimacy was divided into 'good intimacy' (between humans) and 'bad intimacy' (between the human and the machine), and also that the latter was seen as something that potentially threatened the former. While these findings seem dated today, particularly the demonization of coding and computing to such a degree, I think they clearly illustrate how a culture of exclusion has been created alongside the notions of what is appropriate behavior towards the machine, and how these notions are gendered and sexualized. A discursive 
connection was made between computing and sexuality, which created boundaries between sexuality and computing, humans and machines, 'right' and 'wrong' forms of intimacy.

Therefore, Software Studies scholar Ron Eglash asks why "obsessive intensity in writing poetry or communing with nature can be celebrated, but similar focus involving machines should be condemned" (2002, 82). In this way, he almost echoes Ada Lovelace's interdisciplinary call for computing to be seen as 'poetical science'. He argues that there are normative concepts of sexuality, love, intimacy, and technology based on romantic organicism and a Christian cultural legacy that tend to demonize technophilia even today.

Indeed, remembering Berlant's stance that the drive toward intimacy "is a kind of wild thing that is not necessarily organized that way, or any way" (2011, 284), I suggest that also supposedly 'detached' activities such as coding and computing cannot be not affective and never have been. Sexualizing this form of attachment and condemning any kind of sexual attachment, on the other hand, means to localize intimacy solely in a normative space of convention and to render intimacy with and through the machine as 'different', which here means 'bad'.

\section{Going Back and Forth}

This compressed run-through of the history of women ${ }^{78}$ and coding suggests that women's relationship with coding, in tandem with its cultural constructions and inductions, can be understood as happening in

\footnotetext{
${ }^{78}$ Other historical figures who could be mentioned are Grace Hopper, who invented the first compiler for a computer programming language; Margaret Hamilton, who worked on NASA on-board flight software and popularized the term 'software engineering' to underline the importance of thinking about software in the vein of engineering; or Katherine Johnson, Mary Jackson, Dorothy Vaughan, Kathryn Peddrew, Sue Wilder, Eunice Smith, and Barbara Holley, black female computers who worked for NASA in segregated offices and whose histories have only very recently been re-discovered (Shetterly 2016). They are said to have made a major contribution to winning the space race and their shared story has now been adapted for a Hollywood movie.
} 
forced waves of attachment and detachment and that culturally changing notions of intimacy with the machine play a part therein. 79

As alluded to in the introduction to this chapter, Teresa de Lauretis speaks of different 'technologies of gender' (1987) to explain on- and off- moments in the relationship between women and coding. Extending Michel Foucault's analysis of the body, which did not sufficiently take into account specificities of gender, de Lauretis discusses how gender becomes one of the effects of differential social technologies and how they in turn shape gender.

Stories such as Ada Lovelace's, or those of the ENIAC girls or other female icons of computing, have for a long time inhabited positions that were rendered unrepresentable. They were in the 'space-off' or elsewhere and are only now being re-discovered through (feminist) practices. This elsewhere, however, as de Lauretis argues, often takes form through 'counter-practices' and in alternative spaces.

What this small historical review also shows is that, at certain times, discursive spaces have opened up for women in coding, but that these were often tied to features associated with femininity (as emotionality or social skills), in addition to or instead of technological expertise (Corneliussen 2016, 210).

Looking at these waves of attachment and detachment of femininity and coding, one could rather think of a revival, or a falling in and out of love. It is a fact that there were waves of women being close to

\footnotetext{
79 This relates to a particular western context. The idea of women shying away from computing becomes peculiar if viewed from a non-European, e.g. Malaysian, perspective. Vivian Anette Lagesen has explored the question of whether Malaysia is "A Cyberfeminist Utopia" (Lagesen 2008). In contrast to Western societies, narratives such as those relating that computer sciences should be a masculine profession or the imaginary of the male computer nerd are lacking. Instead, computer sciences are perceived as a career path that is highly suitable for women, as Lagesen deduced from interviews with Kuala Lumpur University students and teaching staff. Her final conclusion, however, was that the Malaysian situation is not some kind of 'cyberfeminist utopia', but that it arose more out of practical necessity and economic need, combined with the idea that working in offices (indoors) was more suitable than working outside (for example, on construction sites), and the culturally disseminated idea that women simply like 'theoretical work' (Lagesen 2008, 22).
} 
computing and coding and waves of being far from it, in terms of both physical proximity and cultural ideals. As I want to suggest, these waves also correspond to changing understandings of coding as either an intimate, materialized, embodied, affective, or even poetic experience or as detached, cold, prosaic, or even pathologically sexual.

The question from the vantage point of digital intimacy is thus if a more radical embracing of coding as an embodied, affective practice (as the contemporary women*-coding initiatives, that I discuss in the following, seem to suggest) could be a counter-practice towards 'detachment narratives' that construct coding and computing as cold, anti-social activities, which lack any acknowledgement of its affectivity and instead display a 'chilly culture', as digital culture scholar Hilde G. Corneliussen names it $(2016,210)$.

\subsection{Women*-Centered Coding Groups as an Approach to Revive a Love Story}

With today's omnipresence of digital technologies and media, women's involvement and fascination with digital technologies has risen, accompanied by a growing female presence in accompanying occupational fields (Corneliussen 2016). Furthermore, for many, intimacy with and through digital technologies is generally no longer pathologized; especially since hand-held devices have replaced stationary computers, and since social contexts are understood as being remediated rather than replaced by the machines.

This is also the context for the coding initiatives that I discuss in the following, and which I see as an interesting example of the relationship between women and coding today. For example, Linda Liukas, founder of Railsgirls, explains in an interview:

Spotify is changing music and Facebook [is changing] friendships, as well as Amazon books, Google information, and so much more. It's all about making change [...] and 
my concern is that this change or transformation is driven only by young boys from California in their early twenties with a very narrow world-view. [...] The world of IT engineering has a culture, is a community and also shows empathy. It is not boring or bland. (quoted in: Neubauer $2012,133)^{80}$

Liukas is framing contemporary processes of digitization and their strong embedding within neoliberal markets, their impact on so many aspects of everyday life, and their remediation of well-known media and even relationships as motivation for initiating the Railsgirls network. Her worry is that these changes are happening in a way that is too uniform, and that the missing diversity within the computing industry reflects negatively in various ways, be it on the design of software and or on women's career options.

In this sense, Liukas is sharing an often-voiced critique of male dominance in tech industries, particularly that women and non-Asian minorities are underrepresented in computing and that this is due to a particular geek culture (Hacker, Smith, and Turner 1990; Eglash 2009) which in recent years has extended to app economies. ${ }^{81}$ It has been argued, for example, that 'computer nerd' is an inherently white, male category (cf. Eglash 2002; Bucholtz 2001; Kendall 2011).

Coding initiatives such as Railsgirls strive to do coding/technology differently; that is, to create an atmosphere that is opposed to abstract ideas about coding environments defined by male hackers, whiteness, and working modes favoring solitude and emotional and physical detachment. What are their approaches to achieve this? In the following section, I investigate their use of 'soft' or emotionally charged notions to achieve such different coding environments.

\footnotetext{
80 This translation from German to English is mine.

${ }^{81}$ The critique has often been voiced that tech-industry strongholds a la Silicon Valley are tightly in men's hands, at least when one looks at plain numbers of who are the makers behind social media, the app economy, or other digital startups, and this is often related to sexism in hiring strategies and the workplace (for a collection of experience reports, see Shevinsky 2015; for a survey, see Vassallo et al. 2016).
} 


\subsection{Soft Code Skills}

As pointed out in the context of the documentary CodeGirl, but also evident, for example, in the project name 'Made w/code' (Google's own campaign to motivate girls to code - a wordplay on 'Made with love'), what is striking is the constant underlining of safety, love, community, and other positively connoted intimate phrasings in the campaigning and promotion of many of the initiatives. ${ }^{82} \mathrm{My}$ objects of analysis in this chapter are different coding initiatives that I see as parts of this social trend, meaning that they also stand for the idea that women and girls need to be introduced to coding in 'different' ways, that is within frameworks of intimate notions such as sociality, friendliness and compassion. I am particularly interested in their use of the intimate and other positively connoted emotional phrasings to achieve this different approach.

For example, the US-based organization Girl develop it encourages visitors: "Don't be shy - develop it" on the homepage of their website and promises "affordable and judgment-free opportunities for women interested in learning web and software development." ${ }^{33}$ Similarly, Railsbridge (USA) is "working to make tech more diverse and welcoming by teaching programming, connecting human beings, and listening to people's needs." 84 The Canadian Ladies Learning Code defines their mission as: "to be the leading resource for women and youth to become passionate builders - not just consumers - of technology by learning technical skills in a hands-on, social, and collaborative way." 85

\footnotetext{
82 How are these organizations structured? To give an example, Railsgirls is a non-profit volunteer community which offers (mostly) weekend courses in basic programming to girls, women and everybody else - but girls and women are the target audience and free spots are reserved for them. Railsgirls was founded in Finland in 2010, but the concept has spread worldwide and today tech-savvy volunteers can organize Railsgirls workshops with the help of open-source tutorials and materials

(http://railsgirls.com/materials, last accessed 4/o8/2016). They have a range of sponsors, from small startups to big players.

83 https://www.girldevelopit.com/, last accessed 15/10/2016

84 http://railsbridge.org/, last accessed 14/10/2016

85 http://ladieslearningcode.com/about/, last accessed 14/10/2016
} 
The Women's Coding Collective promises to "cultivate supportive, nostupid-questions environments where women can learn, build, and code together." 86 The Finnish-born network Railsgirls aims "to give tools and a community for women to understand technology and to build their ideas [...] by making technology more approachable." ${ }^{87}$

Some organizations are underlining the diversity factor even more clearly. The explicitly LGBTQI-friendly British organization codebar.io (which also has chapters in Berlin and Barcelona), for example, explains on its homepage that its "goal is to enable underrepresented groups to learn programming in a safe and collaborative environment and expand their career opportunities." 88 Blackgirlscode's vision is " $[t]$ increase the number of women of color in the digital space by empowering girls of color ages 7 to 17 to become innovators in STEM fields, leaders in their communities, and builders of their own futures through exposure to computer science and technology." 89

But apart from the fact that all of the listed initiatives address girls, ${ }^{90}$ women and/or LGBQTI people, what they have in common first and foremost is their emphasis on notions of safety and collaboration, the absence of judgement, and sociality, passion, community, care, or similar notions of positively connoted emotional values.

A conclusion that can be drawn from this is that there is a wish to create 'safe spaces'. 'Safe Space' is a concept originating in the USAmerican women's movement, where it denotes a space which

86 https://thewc.co/, last accessed 14/10/2016 $87 \mathrm{http://railsgirls.com/,} \mathrm{last} \mathrm{accessed} \mathrm{14/10/2016}$ $88 \mathrm{https} / / /$ codebar.io/, last accessed 14/10/2016 $89 \mathrm{http}: / /$ www.blackgirlscode.com/ last accessed 14/10/2016 ${ }^{\circ 0}$ Some of these organizations - particularly those aiming predominantly at younger girls - work with what some critics call 'pinkification'; that is, their homepages work with stereotypically girlish colors such as pink, purple, or pastel colors or feature cute or kid-friendly illustrations, for example, kittens (Railsgirls) and digital pictures and avatars (for example, Techgirlz, Railsgirls, The Women's Coding Collective or Google's Made w/Code project). 'Pinkification' means the use of pink as a cultural signifier for anything feminine, in order to differentiate it from the other (usually unmarked, normal) artifacts. It is a practice that reinforces gender-based stereotypes and differentiates and organizes products away from an assumed norm. 
implies a certain license to speak and act freely, form collective strength, and generate strategies for resistance [...] a means rather than an end and not only a physical space but also a space created by the coming together of women searching for community (Kenney 2001, 24).

To begin with, these places were typically consciousness-raising groups and queer bars, and later online groups and communities. However, this concept seems to have extended or remediated into a context of coding or hacking environments, ${ }^{91}$ similar to how, for example, the concept of trigger warnings in online discussions is a remediation of previous queer, feminist community-building strategies.

Here, the safe space becomes a room for tinkering and playing around with digital technologies. It creates a space where participants do not have to be 'shy' and will not be judged for making mistakes. But, first and foremost, the idea is to give women* the space to adopt a programmer identity, without being questioned or doubted. The hope is that these focal points will help to change the perception of what programing is and will change its cultural parameters, to re-invent coding away from the historically grown idea of technology as cold or non-sensual.

\section{Before the Workshop}

This 'soft' and social way of framing the coding experiences and the historical baggage that has collected around women and coding shaped my expectations before entering a workshop offered by one of the aforementioned organizations in a particular way. I have argued that they are trying to establish something similar to 'safe spaces', which

${ }^{91}$ This is much more obvious in the explicitly feminist hackerspaces that have popped up in the last three years (Toupin 2015). These are not part of my analysis here since I am focusing on the bigger, better-known initiatives. 
build an alternative to the historically developed picture of hacking environments as 'chilly' or inimical to women. Based on this pre-research, two observations stand out.

Firstly, the promotional material of these women*-centered coding initiatives seems to feature a subtext that could be described as taking an approach inspired by 'sexual difference'. Sexual difference, in the sense of French post-structural feminism as popularized by, for example, Luce Irigaray (2004)in opposition to androgyny or gender neutrality within equality perspectives, argues for an understanding and re-evaluation of the ways in which women are different from men. Women's cultural, sexual, and bodily differences and their lived experiences are celebrated and more highly valued (Pilcher and Whelehan 2004, 39).

With their permanent highlighting of women and girls (for example, in the organizations' names), the appellation of the equality-gap discourses, and the subtext of valuing particular, supposedly feminine, skills (for instance, sociality) or weaknesses (such as shyness), it can be argued that a sexual-difference perspective is at least tentatively present.

A second, interrelated expectation is that in these contexts programming is fused with notions of what Antonio Negri and Michael Hardt have theorized as 'affective labor' (Hardt and Negri 2005; Hardt 1999). This notion is based on feminist analyses of women's work ${ }^{92}$ and what they have called "labor in the bodily mode": "Caring labor is certainly entirely immersed in the corporeal, the somatic, but the affects it produces are nonetheless immaterial. What affective labor produces are social networks, forms of community, biopower" (Hardt 1999, 96).

Typical domains in which to recognize affective labor, according to Hardt, are 'services with a smile', such as being a flight attendant or gastronomy service staff, that require pro-social behavior and a friendly attitude.

92 Hardt is exemplary pointing to Smith, Dorothy E. 1987. The everyday world as problematic: A feminist sociology. Northeastern series in feminist theory. Boston: Northeastern University Press. 
In the context of digitalization and social media networks, affective labor is sometimes also discussed as 'digital labor' or 'free labor' for example, when users generate social and affective content (clicking 'likes', posting pictures, writing texts) that is not usually considered to be work, but nevertheless plays into market logics and is monetizable for the network operators (Terranova 2000). The point is that affective labor is also notoriously 'immaterial labor', both in the sense that it does not produce graspable, concrete, or long-lasting results and that it is often unrecognized and uncompensated additional work affecting the worker's body.

When it comes to women*-centered coding initiatives, affective labor plays a role in the sense that the investment in community, sociality, friendliness, etc. is an additional effort, an extra kind of work in the bodily mode - which is more or less implicitly expected from women and girls, both for the side of the organizers who create an affective safe space and from the participants who seek out their courses.

Building on this analysis of women* and coding initiatives, I now turn to my own experiences of attending such a coding group.

\subsection{The HackerWomen's Workshop}

I am in a rush when I arrive at the workshop venue. The last-minute email instructing participants to use the back instead of the front entrance did not reach me in time - I am abroad and do not have internet access on my smartphone (a fact I experience as a big annoyance). It is my second day in the country and I have only just made my way to the regional capital. Having lost a couple of minutes because I was standing in front of the wrong doors earlier, I am now the only one who has not set up her laptop yet. As it turns out, this is not the last time that I will feel as though I am lagging behind on this Saturday.

While I start the machine (a slow, black laptop that I borrowed from a friend), I have a swift look around. There are approximately 40 people sitting at tables in groups of four or so. As expected, most of them are young women, but I also spot few men and seniors. We are 
here because we signed up for the Intro to HTML \& CSS workshop organized by HackerWomen. ${ }^{93}$ Nadine, who is introducing the session as one of the organization's representatives, is giving a quick opening speech, in which she underlines the notion of connectivity; from her perspective, the organizers, participants, and volunteers are becoming part of a community through the workshop.

I sit down at a table with three other young women, all from the capital. The idea is that whilst the teacher of the day, Tanja, is explaining and at the same time implementing the HTML and CSS codes on the big screen, we follow her lead and try to apply the different commands on our own laptops simultaneously.

But before we start, all the mentors introduce themselves.

Of the nine volunteers, only two identify as women. ${ }^{94}$ When asked about their motivation to get involved with the organization, two of the male mentors point out that women use digital technologies in everyday life, but are seldom involved in the making process, and that they feel this is a state that needs to be changed and that girls and women should be "encouraged". In his short introduction, another mentor names his little daughter as I reason why he working as a mentor here. He wishes to have more female role models for her in the technical and engineering fields, and in coding particularly. Overall, we are encouraged to feel like part of a larger whole.

Just as on the organization's homepage, the idea of community or connectivity is emphasized at the beginning of the workshop. The recital of

\footnotetext{
93 As explained in my methodological sections, all names (of the organization, mentors, teachers, and participants), are anonymized.

94 The majority of men within the group of mentors can be explained by the de-facto male majority in IT and related professions. The wish to 'help out women' as a group to enter the field of tech is understandable and likable. On the other hand, I am puzzled about the implications of such a 'helper's paradigm'. For example, I personally noticed that I was slightly disappointed by the fact that I had a male mentor assigned to me, because it was contrary to my expectations of an organization founded and run by women, as the homepage of this organization and those of others underline. It was simply an aspect that I had not expected there, even though he was very nice and helpful.
} 
the 'missing women in tech' narrative confers a political dimension, while the remark from the father who desires more role models for his young daughter introduces an emotional element.

It is therefore obvious that the workshop participants are being appealed to as women in different ways: as being aware of the gendergap issue, as encouraged or in need of encouragement, and also perhaps as being touched by the story of the little girl looking for proper role models. In this sense, I would argue that traditional pictures of femininity remain relatively untouched in this context. In other words, the approach is not so much to change what femininity is in relation to coding, but to use existing culturally dominant notions of femininity to make coding more attractive. Therefore, I think the coding workshop as I experienced it was not very interested in questioning or challenging a masculine/feminine binary per se. One could also say that they were not trying to be queer. In this sense, the hypothesis that, from a feminist theoretical perspective, the women*-centered coding workshop I attended can be located in a sexual difference approach can be partially confirmed. So how about the aspect of affective labor? What follows is another excerpt from my fieldnotes.

\section{Work Life Lunch Talk}

During the lunch break, I have time to talk to the participants in my group (i.e the three other women sharing a table and mentor with me) and step back from the role of participant into the role of an observer or interviewer. Asked about her experiences with previous coding courses and how they differ from this workshop, one of them tells me that she prefers workshops like this one over regular training courses because the latter tend to be more "strict and school-like" - and much more expensive. For her, it thus does not seem to be so much about the community factor. Furthermore, all of the young women from my table tell me that they work in different mid-ranking office jobs and that their curiosity about learning to code is not primarily out of interest in technology per se, but is primarily a necessity: The requirements of 
their jobs are changing. A basic understanding of HTML is increasingly becoming a requirement, "at least I must be able to point out the problem when I see the [broken, H.S.] HTML code”, as one of them explains. I found it interesting that all three women were so similar (for example, in age, gender, nationality, location, qualifications), even though they did not know each other before, and that they all agreed on the motivation for taking this and similar courses: to gain better job skills - and, to a lesser degree, 'for fun'. But none of them was hoping to start their own online project.

What is peculiar about the women coding trend is that most of these initiatives are embedded within the tension between feminism and neoliberalism. On the one hand, they re-cite feminist statements and strategies (without, at least to my knowledge, ever naming them as such), but on the other hand, seem to follow particular rules of the neoliberal market at the same time.

As a hard-to-pin-down concept that is sometimes disputed for being too thinly-stretched to maintain usability, the term neoliberalism is generally used to summarize political and economic practices that engulf differing takes on free markets, free trade, and their implications. These are typically "achieved through economic deregulation, elimination of tariffs, and a range of monetary and social policies favorable to business and indifferent toward poverty, social deracination, cultural decimation, long term resource depletion and environmental destruction" (Brown 2003, 1).

Furthermore, as political scientist Wendy Brown has established, there are impacts on the organization of subjectivity, meaning that subjects become 'entrepreneurs' in neoliberal settings: They have to work on themselves, they are responsible for their own optimization, they have to make themselves attractive to an always-demanding (job) market (Brown 2003). This becomes even more true for women, because neoliberal markets tend to address them more thoroughly (Scharff 2014). "It is mainly women who are called on to transform themselves, which becomes particularly visible with regard to the management of the body and sexuality" (Scharff 2014, n.p.). 
Christina Scharff's empirical work on the entrepreneurial subject, for example, points towards the downsides that become noticeable for many women in the workforce: social critique becomes self-critique, gaining self-confidence sometimes is achived by othering (e.g. women in victimized positions or those who are simply considered not to 'try hard enough'). Therefore, Scharff concludes, becoming an entrepreneurial subject often comes at a price: "neoliberal subjectivity seems to evoke a range of affective states that are linked to anxiety, depoliticalization, the repudiation of vulnerability and an internalization of competition" (2014, n.p.). It can therefore be seen as being at odds with its self-proclaimed empowerment narrative.

What are the implications of this for the coding workshop that I discuss? Here, the participants' interest in this workshop seems to be almost exclusively based on following a particular demand that is actually a requirement from their workplaces, to the extent that they are prepared to spend both money and an entire Saturday's worth of time attending a workshop.

\section{Work as Affective Impasse}

Journalist and author Susan Faludi has criticized similar forms of conflating empowerment narratives with entrepreneurial agendas. For example, she sharply criticizes Sheryl Sandberg's 'Lean In' feminism, or what she calls 'Facebook Feminism' (Faludi 2013). Sandberg, currently chief operating officer and one of the very few women at the top of $\mathrm{Fa}$ cebook's management, has published Lean in: Women, Work, and the Will to Lead (2015) as a guidebook for modern working women. Its philosophy has propelled it from a bestseller to a global network with a dedicated online community and sold-out live performances.

Lean In constitutes Sandberg's call to women to overcome inner obstacles in order to thrive in the workforce: for example, to overcome the fear of asking for a raise or promotion, because one is too shy or it is 'inappropriate' for a woman. Faludi argues that 
Sandberg's admirers would say that Lean In is using free-market beliefs to advance the cause of women's equality. Her detractors would say (and have) that her organization is using the desire for women's equality to advance the cause of the free market. And they would both be right. (2013, n.p.)

Faludi's critique is that 'Facebook Feminism' is not advocating for structural, social, and economic change, but is trying to push individuals to fight harder for themselves and thereby neglecting the historical relationship between labor and women's rights; that they are not fighting institutionalized discrimination but encouraging women to accept accelerating career demands - often without the proportional benefits. The Lean In strategy, as Faludi describes it, is making "demands of individual women, not the corporate workplace" (2013, n.p.), and she argues that some corporate partners of the organization use it to 'whitewash' their otherwise discriminatory company politics.

This critique could similarly be applied to the women coding initiatives as they are introduced here. Instead of having a clear feminist agenda and calling for social change, there is a risk that the burden of fighting for this change will be placed solely onto individual women's shoulders. How is that happening in the workshop?

The workshop participants to whom I talked face increasing demands in their working lives, which come in tandem with processes of digitalization. This resonates with how Melissa Gregg speaks of the "potential of the 'always present', a new form of affective labor that must be constantly regulated" $(2011,3)$. The fact that participants (often) spend money and their free time to adjust to these demands reflects Melissa Gregg's notion of "presence bleed" $(2006,2)$, which describes a multitude of symptoms that make work and life 'bleed' into each other more extensively in workplaces that depend on digital technologies and media.

This, she argues, often happens to the detriment of intimate and social relationships; for example, when work moves out of the office, not only into cafés or train compartments but also into the private 
sphere of homes, onto kitchen tables or into the bedroom. Or when work hours become more fluid, as people work after office hours, are on call at the weekend, or check their emails around the clock. All of these symptoms of professional 'presence bleed' dissolve the firm boundaries between personal and professional identities, increasingly affect intimate lives, and are in the interests of a neoliberal market logic. Learning to code in one's free time in order to be able to keep up with the job and doing it with a kind of affectionate passion could thus be seen as immaterial, unpaid work.

Elsewhere in my fieldnotes, I wrote:

During the course of the day, I hear a general admiration for programmers and coding professions from several participants. It is considered by many here to be something extremely good and valuable: there is no doubt that being a programmer is a very admirable, honest job with role-model character and the potential to change the world. Two other participants I talk to in the lunch break enthuse about success stories; they have heard of a friend's friend who works at Google, who 'has made it'. This individual gets to work in this dream place with free healthy lunches, childcare, and napping corners. One of the young women cites the recent Hollywood movie 'The Internship' as something she can relate to.

This light-hearted comedy from the year 2013 tells the story of two middle-aged salesmen who cannot keep up with the structural changes in their profession that have been introduced through the digital age. Therefore, they apply to Google for internship positions, and despite being in direct competition with young, tech-savvy applicants and being hopelessly unqualified, they succeed and get the posts.

Regardless of the movie's unrealistic plot, the two young women who brought it up when I was talking to them could identify with the situation of feeling as though one cannot keep up.

It might be useful to understand these tensions in relation to what Lauren Berlant has called 'cruel optimism'. In her book of the same name, she defines cruel optimism as a relation in which something that 
is desired "actually is an obstacle in your flourishing. It might involve food; it might be a fantasy of the good life, or a political project" (2011, 1). It is, in other words, something that is adopted in order to improve one's life, like taking up a new habit, with the hope that it will bring someone closer to a 'good life' while actually the person is in a neoliberal impasse and the perceived improvements are only adjustments to this impasse.

The idea of a good life, a "moral-intimate-economic thing" (2011, 2), is fueled by fantasies of upward mobility, durable intimacy, romantic love, job security, and political or social equality. To Berlant, optimism is "not a map of pathology but a social relation involving attachments that organize the present" $(2011,14)$. It manifests "in attachments and the desire to sustain them" $(2011,13)$. In other words, from the vantage point of Cruel Optimism, the ordinary becomes a situation, "an impasse shaped by crisis in which people find themselves developing skills for adjusting to newly proliferating pressures to scramble for modes of living on" $(2011,8)$.

In this sense, the movie The Internship and the stories of successful careers in digital industries might nourish such a fantasy. This "affective structure of an optimistic attachment" makes us believe that "nearness to this thing will help you or a world to become different in just the right way" (Berlant 2011, 2, emphasis in original), while it is more likely that the extra work and affective work done in the attempt to develop new skills is merely an adjustment to the impasse.

\subsection{Discussion: Coding, the Soft Way?}

In this chapter, I have discussed different women's coding initiatives ranging from an app competition, through an encouraging documentary and the re-appropriation of female coding icons, to coding courses and workshops - as a societal trend that has emerged in recent years. A situated look back through history showed that the relationship between women and coding did not initially need this kind of soft push (since there were pioneering women in the field who did not consider 
that technology was 'not for girls'). Furthermore, it retraced the cultural parameters influencing 'Off' moments in the On/Off love story between women and coding; for instance, the construction of the programmer as detached, male, and white. I argued that gendered, normative notions of 'good' forms of intimacy (i.e. not with the machine) played a role in this as well.

This might be one reason why present-day women's coding initiatives seem to include an interest in providing 'safe spaces' to counteract constructions of coding experiences as non-sensual, cold, detached, or solitary, and as a male domain. Their counter-measure, it seems, is to show that coding can be a different activity: social, communicative, and fun. Rather than detachment, attachment to each other seems to be the goal.

As I have illustrated, this approach opens up some spaces for novel female programming identity formations, but ultimately it does little to question, challenge, or change the existing gender binary. On the contrary, there is even a risk of cementing the idea of innate differences through the appellation of supposedly feminine values that are consequently juxtaposed against supposedly masculine (coder's) traits.

In this sense, the coding workshop that I visited in particular, and many other variations on the women's coding trend in general, seem to be a bit of a mixed bag. They have reactive and generative sides; reactive in the sense that they are responding to the gender gap with a critical-mass approach that might perpetuate existing gender categorizations, and generative since they do, after all, have at least the potential to open up particular spaces for female coding identities.

A post-structural understanding sees gender as more fluid than previous definitions, as in Judith Butler's assessment of gender as performative. Here, gender is a 'doing' rather than a 'being' (Butler 1993, 25), and is shaped through iterative interactions within a "highly rigid frame that congeals over time to produce the appearance of substance, of a natural sort of being" (Butler 1993, 33). In the same way, technology and gender identities are today often seen as shaped together and as shaping each other. They are both "products of a moving relational 
process, emerging from collective and individual acts of interpretation." (Wajcman 2010, 150). For Judy Wajcman, this is a more nuanced way to capture an increasingly complex relationship between gender and technoscience, "over time and across multiple sites" (ibid.).

My suggestion is thus that the women's coding initiatives could benefit from an understanding of the relationship between gender and technology as a more mutual one or, if you wish, from understanding the love story as not being unilateral. It is not only the women who need to change or adapt (as the women's coding initiatives promote), but also the technology and the corresponding socio-technical networks are, and should be, in a moving, relational process at the same time.

One way to shift the perspective away from tutoring women into 'softly' adapting to changing techno-economic demands could be to change the idea of intimacy or the use of intimate notions. While the idea is to create intimacy through appealing to notions of community, solidarity, and sociality, this only seems to work to a certain degree. As I have suggested within the conceptualization of digital intimacy, and drawing upon Berlant (1998), the insight is to see intimacy not only in normative terms, for example as sexuality, human closeness, or kinship, but as all kinds of affective attachments. Thus, as digital media theorist Jussi Parikka proposes, keeping in mind the "weird materialities of network culture that relate to movements across scales and between human and nonhuman agencies" $(2015,110)$ could be a way to reframe intimacies with digital cultures. The point is to extend the idea of intimacy to the affectivity of digits, devices, and overall sociotechnological networks.

This take also profoundly mirrors my personal workshop experience, which was characterized by the constant switching back and forth between large (the teacher's) and small (my) screens; by the interaction with the mentor and my workshop colleagues; by being hyperaware of how the keys of the borrowed laptop felt somehow different under my fingers; by an increased cortisol level due to the newness and rapidity of the coding instructions, and later by the concreteness of the result, the homepage I created for myself. 
To give an example: Parikka has analyzed different software-based art projects as a way to understand software cultures differently, i.e. as more relational, affective, and phenomenological $(2015,106)$. Printing or orating code (as is done in the art performances he discusses), for example, means to take code out of its usual context, and to make it comprehensible in a different way. However, it is still difficult to process the information when code is printed or orated:

The challenge of such an approach is to consider things not immediately available to the senses as completely real. Code is one of those things whose scale is slightly at odds with the normal symbolic processes humans have used to operate culture, for instance writing, even if having a relation to it. Code operates at different speeds, and on such a distributed level that it seems to escape simple definitions. (ibid.)

Having code spelled out in an experimental manner - not in the framework of software art, as Parikka describes in his text, but nevertheless in an atypical way during the workshop experience - allows the generation of a different perspective on what code can and cannot do. At HackerWomen, the focus is on how code can be used collectively to create communities, which can be seen as one approach to making code more 'available', by translating it into the realm of sociality and compassion.

My point of critique is the use of these intimate notions (passion, sociability, softness) within a framework of normative ideals (women as bearers of particular 'soft skills'). The way in which this is done seems to challenge or transform traditional perceptions of gender only to a limited degree. As suspected, there is a tendency to try to upgrade or reevaluate supposedly feminine values and skills, mirroring sexual difference positions. This is evident in the frequent appellation of a women's community and the underlining of supposedly feminine soft skills. By implication, this might also contribute to exactly what it 
wants to avoid, the co-creation of a generalized, masculine hacker figure at the other end of an imagined spectrum. In this sense, the myth that makes some young women shy away from coding might live on.

In other words, I am suggesting that a different perception of intimacy - which I have named digital intimacy - in the context of women's coding initiatives, could be a more valuable starting point for coding initiatives of this kind. This would see engagements with the digital in the form of much more mobile attachments, as more open assemblages of social technical networks with all their materialities.

Such an approach would leave open a space for understanding gender as a 'doing' rather than a 'being' (Butler 1993, 25), and for technologies and gender to shape each other in "a moving relational process, emerging from collective and individual acts of interpretation" (Wajcman 2010, 150). Such a shift in perspective might disentangle the participants from historically developed and culturally constructed ideas of coding, computing, and the digital as neutral, emotionally vacant, and somehow immaterial, without having to evoke implicit cultural ideas of what might make coding more attractive to women. 



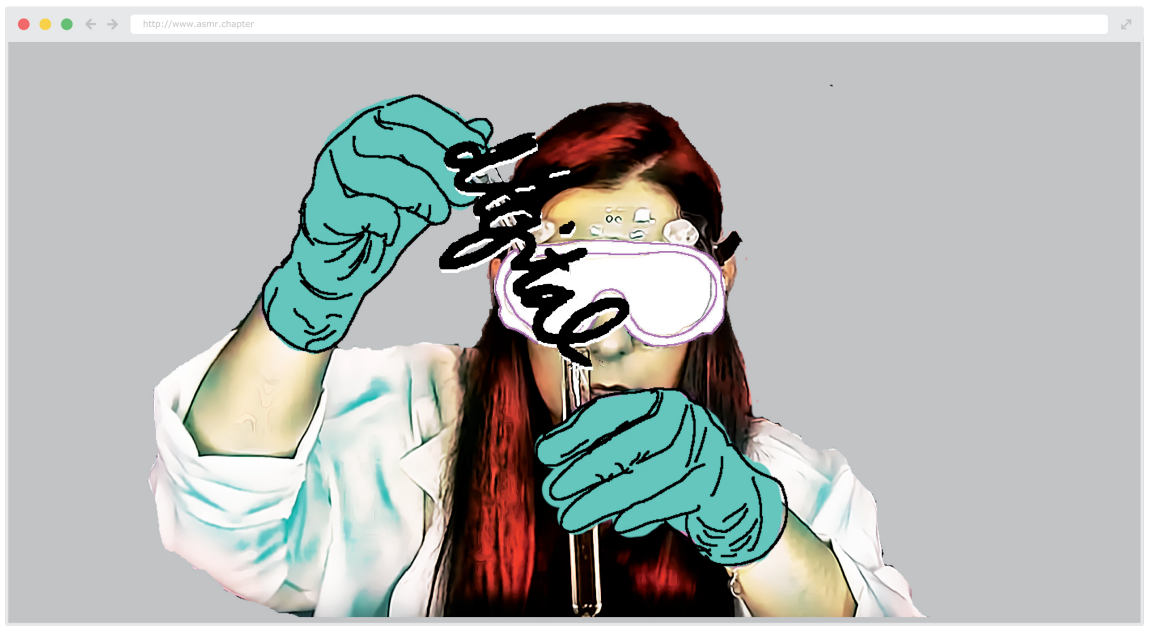

\section{FRIENDLY FACES IN DIGITAL PLACES EXPLORING THE WORLD OF ASMR VIDEOS}





\section{Friendly Faces in Digital Places: Exploring the World of ASMR Videos}

In this final empirical chapter, I am turning to the subculture of ASMR ('Autonomous Sensory Meridian Response'), an idiosyncratic example of visual and multisensory exchanges of intimacies, as it is predominantly celebrated on Youtube. Here, I analyze how intimate videos not always have to stay in the logic of the 'male gaze' (Mulvey 1989) but actually can transform viewership, question the boundary between online and offline, and ultimately open up spaces for the women of ASMR to gain heightened levels of control of their representation. Finally, I also discuss what reverberations such a form of digital intimacy might have on a broader societal scale and particularly in a neoliberal context.

\subsection{What is ASMR?}

"And... they are very soft. It feels very nice... against your skin. Do you agree?" YouTube starlet VeniVidiVulpes is asking me about the bristles of her make-up brush. It is the fifth minute of a 34-minute YouTube clip. 95 So far, the young woman has introduced herself and described the brush. All of it in a slow, whispering, calming, and somewhat seductive voice. She has stroked the bristles over her cheeks, nose, and temples plenty of times, all whilst describing the advantages of the tool and its feel on her skin. The moment in which she asks me, the viewer of the video clip, about my opinion on the feeling, however, comes as a surprise. She strokes the brush around the edges of the lens, trying to give me the impression that she is actually touching my face with the

95 https://www.youtube.com/watch?v=m-lHoJzlrJU, last accessed 15/10/2016. 
brush while looking at me with her big brown eyes. I can hear the bristles scratching the camera's microphone. Going one step further, she says: "The brushes also smell like vanilla", holding the specimen towards the camera. "Here. Do you like it?" She smiles and continues stroking her face. In the seventh minute, she comes closer to the recording device so the viewer gets to hear the sound of the bristles better. Then there are silences. Strokes on her face, strokes on the lens, around the lens. Later on, she changes brushes, stroking the hollows of my cheekbones. Silences, smiles, the sound of bristles against skin. Other small brushes, bushy brushes, thin brushes. A lot of stroking. For more than half an hour.

For an outsider, watching ASMR videos for the first time can be a very peculiar experience. It might be difficult to understand what the purpose of the video or the intention of its performer is. Is it a quirky version of online porn? Is it a sleeping aid, considering how long and apparently uneventful such a clip often is?

ASMR videos might be none and all of these things at the same time. But what is clear is that the clip of the girl with the make-up brushes has been a hit for many ASMR video fans. Lord Lucas comments: "It feels like I've got a thousand spiders crawling all over my head...", play time declares it "the best ASMR on YouTube", while Lisa asks: "Do you take requests? If you do, could you do a roleplay, for example a caring friend?"

The term ASMR is itself a neologism. It stands for Autonomous Sensory Meridian Response, which is a 'new' or 'newly discovered' human feeling and/or physical reaction or sensation. More precisely, it is a "perceptual phenomenon characterized as a distinct, pleasurable tingling sensation in the head, scalp, back or peripheral regions of the body in response to visual, auditory, olfactory, and/or cognitive stimuli." ${ }^{66}$ Nevertheless, the term ASMR is used to capture a number of different ways in which people react to these triggers, not only the 'tingling'.

$96 \mathrm{http}: / /$ en.wikipedia.org/wiki/Autonomous_sensory_meridian_response, last accessed 30/10/2013. 
According to Jenn Allen, who is credited with coining the term, ${ }^{97}$ 'autonomous' refers to the "individualistic nature of the triggers, and the capacity in many to facilitate or completely create the sensation at will" (quoted in: Cheadle 2013, n.p.), thereby underlining the fact that ASMR is not for everyone, but is a highly individualistic symptom that is consequently difficult to prove, narrow down, or classify. The ability to reproduce the response at one's own will, on the other hand, somewhat contradicts the notion of autonomous: Is the response autonomous, a 'wild thing' in the sense of Berlant's mobile intimate attachments, not necessarily organized in any conventional way (Berlant 2011, 284-85), or is it something that can be willfully evoked and follows particular conventions?

The word 'sensory' underlines that it is about sensory perception, meaning that signals are received by the nervous system which result from the affective stimulation. 'Meridian', according to Allen, is supposed to be a euphemism for 'orgasm', presumably in the sense that it refers to sensations that can wander in a particular way through the whole body and induce a lot of pleasure for some people. $9^{8}$ This is also somewhat contradictory since ASMR elsewhere, frequently and often vehemently, is defined as non-sexual or at least non-genital.99

97 Jenn Allen is the founder of asmr-research.org, a group of self-organized ASMRers which is trying to kick-start research by collecting video trials and questionnaires from volunteers, in order to ultimately get funding for physiological monitoring, health screenings, controlled brain profiling, controlled profiling of serotonin and other neurotransmitter levels, psychological profiling, and social-anthropological research in relation to ASMR (last accessed $8 / 10 / 2014)$.

${ }^{88}$ I could not find a reference to Chinese medicine, in which Qi energy is believed to flow through particular meridians, which exist in corresponding pairs and provide guiding lines for acupuncture points. But the association seems not to be too far-fetched and might be implied.

${ }^{99}$ See for example this definition on reddit.com, where the ASMR community has one of its biggest bulletin boards: "Autonomous Sensory Meridian Response (ASMR) is a physical sensation characterized by a pleasurable tingling that typically begins in the head and scalp, and often moves down the spine and through the limbs. Also known as: AIE (Attention induced euphoria), or simply 'head tingles'. This is sometimes referred to as head orgasms, but this is about as sexual as saying eating chocolate is orgasmic (in that it's not sexual). This physical phenomenon isn't experienced by everyone. If 
The word 'response' is self-explanatory according to the neologism's creator, but it could also be suggested that it is borrowed from psychological language, in which a response is understood as a stimulus that constitutes the basis for perception. The idea of naming the 'feeling with no name', as ASMR was previously described in various internet communities, was to make it sound official: "Try explaining why you want money to study 'goose looping' or 'brain orgasms", Allen said in an interview (Cheadle 2013, n.p).

In recent years, the number of ASMR video clips has skyrocketed. On YouTube alone, a search engine request in March 2014 suggested "about 1,320,000 results", ${ }^{100}$ rising by November 2015 to "about $2,460,000$ results". Even though it is not possible to check whether all these videos actually display ASMR videos, and what counts as an ASMR video lies in the eye of the beholder (as the common distinction between 'intentional' and 'unintentional' suggests ${ }^{101}$ ), it can be argued that YouTube is the main outlet and thus the number one address for ASMRers.

Indeed, judging by this huge number of videos, ASMR seems to be a rather large phenomenon. It might be quirky and niche, but at the same time it marks a contemporary practice of everyday internet use and thus is a part of current digitalization processes.

ASMR videos are "intimacy generators", to borrow a phrase used by Martin Stempfhuber in the context of his analysis of the dating app grindr $(2014,49)$. He used it in order to argue, firstly, that digital media can generate genuine intimacy and, secondly, that this kind of intimacy does not lead to a vanishing of the body or of physical closeness

you've never had it before, you most likely won't feel it from the different triggers in this subreddit. NOT TO BE CONFUSED WITH MUSIC BASED TINGLES/SHIVERS. Those are called frisson and can be discussed in /r/frisson" (http://www.reddit.com/r/asmr/, last accessed 25/11/2013, my emphasis).

$100 \mathrm{http} / /$ www.youtube.com/results?search_query=asmr\&sm=3, last accessed 2/12/2015.

${ }^{101}$ An unintentional ASMR video could be something like a Reiki instruction video, from which people experience the response when they see somebody else being touched. Or, of course the Bob Ross videos, since he wanted to teach painting, not give people 'braingasms'. 
$(2014,49)$, both points that are often raised by critics and that mirror digital dualism. In other words, and to stay in the framework of digital intimacy, it can be argued that intimacy is remediated into a very particular format.

As mentioned before, the neologism ASMR was coined in order to describe a particular 'tingling in the head', but what is classified as ASMR on YouTube and the motivation for people to watch these videos is not limited to this particular sensation. In order to begin my analysis, I thus suggest three different categories of ASMR videos, which often merge or overlap. One I would like to call ASMR videos that try to confer 'romantic intimacy', where the performer treats the viewer like an intimate partner or friend. Here, the viewer is often addressed in an uplifting and caring way, for example when TheOneLilium tells the viewer: "I hope that it's ok with you that I'm gonna pamper you a bit 'cause... you are really the apple of my eye."102

Another category, clearly also related to close personal attention, is that of 'intimate treatments' of all kinds: doctor's appointments, eye examinations, cosmetic treatments, haircuts, or a memory-erasing roleplay. ${ }^{103}$ Finally, there is a category that is not predominantly related to roleplay but is focused on repetitive, calming sounds or activities, like painting, woodcarving, or fingers tapping on glass. I call this category 'repetitive relaxation'.

I would like to suggest that these video clips can be understood as displays of digital intimacy in which questions of embodiment, materiality, and digital dualism are vividly renegotiated. Particularly in the role-play videos, for example, when VeniVidiVulpes extends her brush beyond the screen, touching the viewer, who in return gets 'the tingles', interesting questions are raised: what does this tell us about online/offline boundaries, human-machine interactions, on- and offline embodiment, intimacy, and touch 2.0?

$102 \mathrm{https}: / /$ www.youtube.com/watch?v=xBkGaMA9jTo, last accessed 15/10/2016.

${ }^{103} \mathrm{~A}$ re-interpretation of the memory erasure procedure in the Hollywood movie Eternal Sunshine of the Spotless Mind (Gondry 2004). 
Secondly, I will elaborate on the gendered dimension more specifically. Even though, with the multitude of ASMR clips that are available, it is clear that not all of them are from or about young, pretty, white, able-bodied, English-speaking women, it is this group who, firstly, create the majority of the videos and, secondly, are the ones who are most appreciated (in the sense that they have the most views and biggest fan bases), and are almost the only ones discussed in news reportage (Cheadle 2013; Hudelson 2012; Graff 2013) or interviewed for TV formats (cf. Hockridge 2013).

In other words, the 'whispering women', as they have been nicknamed by reporters due to the low, calm, and seductive voices they commonly use (cf. Graff 2013), are the flagships of the ASMR movement. ${ }^{104}$ But can whispering here also mean having a voice? Should ASMR videos preliminarily be understood as a re-installment of the 'male gaze', in the sense of Laura Mulvey's staple in feminist film theory, or are these affective ways of 'doing digital media differently' counteracting a passive "to-be-looked-at-ness" $(1989,122)$ ?

Lastly, I would like to discuss how such a phenomenon can be understood from a broader societal perspective. The ASMR subculture became famous as a YouTube sensation. A recent internet article on the subject even argues that "a bunch of YouTubers discovered a tingling sensation nobody knew existed" (Abbruzzese 2015, n.p.). It can be said that the ASMR subculture is potentially encouraged by digital media, particularly by the popular video-distribution platform YouTube. Thus, the question is also about the impact of digital media on the rise of ASMR - what role does the digital play here or, to be more provocative, would ASMR exist if was not hyped by digital media? And, by extension, what does it say about some people's relationship to intimacy, when affection becomes something that is potentially easily available and consumable, something that is almost indefinitely reproducible through digitality?

${ }^{104}$ I have encountered queer mermen, whispering street dealers, manly men doing handiwork, people of color in diverse subgenres - only a few, but they are there. 
Next, I will approach ASMR videos as what they seem to be at first sight: Videos or short films featuring pretty women in close, intimate ways. While this is only one part of the puzzle, I am drawing on film theory, and more particularly on the feminist film theory evergreen of the "male gaze" (Mulvey 1989). My assumption is that many of the whispering women often break patterns of simply 'being looked at' by encouraging bodily relationships with the viewer via multisensory stimuli, by creating their own narratives and deconstructing the superiority of vision as the prior sense, or what is sometimes called Cartesian perspectivalism (Jay 1993).

An example is the one-hour virtual haircut video from Heather Feather, a treatment that has been watched/experienced almost 960 ooo times. ${ }^{105}$

\section{Meeting Heather Feather}

Following the categorization of typical ASMR videos mentioned above, I would classify VeniVidiVulpes's ASMR video focusing on brushes and brushing as a mixture of 'repetitive relaxation' (the constant and calming brushing of her own face) and an 'intimate treatment' clip (she is directly reaching out to and acting on the viewer's face to treat them with the brush). Other ASMRtists take the genre of the treatment video up a notch. There are highly elaborate clips of air-space hostesses talking the viewer through a trip to Mars, ophthalmologists calmly examining eyes (while holding the flashlights towards the camera to shine the light into the patient's eyeballs), and a burgeoning sub-sub-genre of haircut videos that focuses more on the treatment factor.

This particular video opens with Heather Feather in front of the camera's eye in what seems to be a kneeing or flexed position (in order to come down to me, the viewer, because I am sitting in a lower position as is typical when in a hairdresser's chair). I see her face, her head and the shoulder area in a frontal close-up. It appears as though she is s6VDWc, last accessed $30 / 10 / 2016$. 
less than one meter away. Behind her, in the background, is a black shelf which holds a selection of accessories that might well be found in a hairdressing salon: neatly rolled, white towels; bottles of various shapes and sizes which look as if they could contain shampoo, conditioner, and hairstyling products; and a flower arrangement.

Heather Feather convincingly performs the role of a hairdresser and it is clear that she has thought about her script. Starting out with small talk about her own salon that she would like to open one day and the kind of haircut she might give me, and suddenly, after a hard cut in the video, she is standing behind me. Subsequently, and for long periods of the clip, only the shelf is visible and all that remains of Heather Feather is her voice ("You have... nice hair"), the sound of clipping scissors next to my ears, the sound of her fingers going through my hair, splashing water and scrubbing while she is shampooing my head (I am now in a leaned back position - which means I am now predominantly seeing the ceiling).

One extraordinary aspect is that Heather Feather has put a lot of effort into making the best use of her binaural microphone to create a 3D effect. ${ }^{106}$ When I listen to the video with my headphones on (as recommended), I really have the feeling that some sounds are coming from behind me, from the right side, or from behind my left side respectively, for example when she is shampooing either side. The video takes almost one hour and as well as shampooing, it also features hair brushing, gentle (almost humming) hair drying, the use of spray bottles (“This stuff smells amazing... here, wanna smell?"), a few snippets of small talk ("You're heading out for dinner after this?") and of course a lot of snipping sounds, close to my ears.

\footnotetext{
${ }^{106}$ Binaural microphones use two microphones to create a 3D stereo sound sensation with the goal of conveying the impression of being in the same room as the recording person and their sounds. Interestingly, some binaural microphones are designed to look like human ears, with an approximate head-width connection between them (for example the brand 3 Dio, http://3diosound.com/index.php, last accessed 3/12/2015). ASMRtists having such a device thus speak into ear prosthetics.
} 
Even though I do not feel ASMR, in the sense that I do not get the 'tingles', I am drawn into the world that Heather Feather has created for her viewers. Carefully, confidently, and with a love of detail she has recreated the looks, sounds, postures (of herself and myself), gestures, tactile sensations, and smells of a hairdressing salon and thereby created a moment of closeness and intimacy between a fictional hairdresser and me. She does this for example by pointing out the smell of the hairstyling products or by asking me if the heat of the dryer is too high.

Receiving a haircut is an intimate event - someone who willingly lets another person touch their hair and head, to a certain extent is also at the hairdresser's mercy. At the same time, Heather Feather's performance remains an intimate treatment mimicking another intimate treatment, acted out by a young woman who is beautiful to look at. Does this mean that ASMR videos are mainly about visual pleasure and objectification? In the following section, I will draw on film theory, because it helps me to understand how ASMR videos are different from, and more intimate than, many other viewing experiences.

\section{Watching ASMR}

What is happening here from a feminist film theory angle? It seems a little bit unusual to try to capture something as fragmented, homemade, and usually short as YouTube clips within film theory, and many ASMR fans would argue that the affect is much more about sonic stimuli (like the sound of scissors clipping hair) than about visual effects/affects. However, the visual dimension cannot be dismissed here and film theory delivers useful approaches to thinking about how the film is affecting and embedding the viewer into the occurrences.

A movie is supposed to draw the viewer into a major storyline, which is usually orchestrated by a cast of writers, directors, actors, cinematographers, editors, and so on. Be it a short movie of only a few minutes or an evening-filling blockbuster, the film industry has traditionally created narratives that present a different world and draw the 
viewer into a suspension of disbelief. As has been much discussed by film theorists, this happens with the help of the cinematic apparatus, in the sense of sitting in a dark room, staring at a bright projection which encourages a voyeuristic play (Kaplan 1987, 42).107 From a feminist or gender-studies perspective, the question of the gaze becomes the most prevalent, because it is a first impulse to regard the ASMR women's performances as predominantly responding to a (male) gaze. ${ }^{108}$

Laura Mulvey's iconic essay on the 'male gaze' can be located within the context of film apparatus theory (1989). She structured her argument around two aspects of how sexual difference organizes visual pleasure. Just as in the Lacanian mirror-stage, when a child identifies with a perfect mirror image and falls in love with it, there is a narcissistic desire to identify with the stronger, more complete, and powerful character or the superior viewing perspective, instead of the distorted or empty position of the woman (who exists only in relation to the man on screen).

The second aspect is the omnipresence of the male character, who carries look and action. This is achieved by how camera, narrative, and editing work together as a triple gaze to make the woman a 'spectacle'; she is in the position of "to-be-looked-at-ness" (Mulvey 1989, 122).

${ }^{107}$ Apparatus film theory was popular in the 1970 s to mid-80s and based on post-Marxist ideology and psychoanalysis. The apparatus in apparatus film theory, following Jean-Louis Baudry (1975), sees cinema as an ideological state apparatus which constitutes the viewer in a particular way. From a semiotic perspective, the focus is on camera work, editing, and other film-making techniques. The psychoanalytical perspective contributed new concepts such as subjectivity, desire, and visual pleasure; answering the question of how films can have such a strong (libidinous) attraction with psychosexual reasoning and by comparing the viewer-screen relationship with the Lacanian mirror-stage (Angerer 2015, 5).

${ }^{108}$ After conference presentations on ASMR, participants in their comments often described this as one, if not the defining, aspect of the ASMR phenomenon from their point of view. 


\section{Touching ASMR}

It can thus be said that, in early feminist film theory, there was not much pleasure to be drawn for the female viewer and the assumption was that she could only turn to experimental film or 'counter cinema' (Smelik 1995, 71). Therefore, its theories are today often seen as being too dependent on context and historical setting, in the sense of not accounting for (today's) heterogeneity and the complexity of visual cultures (Smelik 1995, 71).

Later film theory (in the 1990s) became more concerned with the moving body (also in relation to the rise of video gaming and bodies as playing bodies (cf. Angerer 2015, 7). In Vivian Sobchak's phenomenological film analysis, on the other hand, the body is awakened by the film and entangled with its world, which means that it is experienced as a multisensory representation of an objective world and is no longer reduced to an object of vision (1992).

Like Sobchak, multisensory media scholar Laura Marks stresses that film viewing is a corporeal practice and thus challenges ocularcentrism in favor of more corporeal and affective approaches. For my analysis of ASMR videos, Marks' concept of haptic visuality is of particular importance (2002). This offers a different framework for studying the body's involvement in digital media in relation to multiple senses, affect, and embodiment (she is referring in particular to media artwork, ranging from cinema to video to websites). She offers this concept in order to "warm up" post-Enlightenment preferences of disembodied knowledge production, based on a privileging of vision, and aims "to restore a flow between the haptic and the optical that our culture is currently lacking” (Marks 2002, xiii).

She is proposing a tactile way of seeing, a way that involves the body of the viewer more directly, in which the eyes metaphorically become organs of touch. Amongst others, her understanding of the haptic 
draws on Deleuze and Guattari's conception of the smooth and the striated (1988). ${ }^{109}$

While the striated in Deleuze and Guattari's thinking is regulated and partitioned space, which does not allow for movement, their term smooth space represents disorganized matter. In this thinking, smooth space exists in relation to striated space. They co-exist and sometimes happen at the same time. In this sense, the striated relates to the more linear sense of the optical: "It seems to us that the Smooth is both the object of a close vision par excellence and an element of the haptic space (which may be as much visual or auditory or tactile)" $(1988,544)$.

Taking this into account, Marks now proposes: "The haptic critic, rather than place herself within the 'striated space', of predetermined critical frameworks, navigates a smooth space by engaging immediately with objects and ideas and teasing out the connections immanent to them" (p. xiii). Here, haptic and optical visuality are not opposites, they are two ends of one spectrum and slide into each other. The erotics of haptic images (which in her understanding mean a certain loss of control) are related to this sliding along a spectrum, according to Marks, which also means that no media artwork can always be only haptic.

What Deleuze and Guattari have called close vision as part of the smooth - which can also be visual, auditory or tactile - comes together in Marks' haptic visuality, where "eyes themselves function as organs of touch" (Marks 2002, 2). Haptic images, then, for Marks are often marked by being distorted or grainy, because they are too close. In this way, they highlight an inability to see clearly, so that the view needs to

109 "Smooth space is filled by events or haecceities, far more than by formed and perceived things. It is a space of affects, more than one of properties. It is haptic rather than optical perception. Whereas in the striated, forms organize matter, in the smooth, materials signal forces and serve as symptoms of them. It is an intensive rather than extensive space, one of distances, not of measures and properties [...] Perception in it is based on symptoms and evaluations rather than measures and properties. That is why smooth space is occupied by intensities, wind and noise, forces, and sonorous and tactile qualities, as in the desert, steppe, or ice” (Deleuze and Guattari 1988, 479). 
become more haptic. This then, according to her, is exactly the way in which

haptic images have a particular erotic quality, one involving giving up visual control. The viewer is called on to fill in the gaps in the image, engage with the traces the image leaves. By interacting up close with an image, close enough that figure and ground commingle, the viewer gives up her own sense of separateness from the image. $(2002,13)$

A similar letting go of the separateness of the image might come into play when closeness is acted out as a digital touching beyond the screen in ASMR videos. Even when the pictures are not grainy (even though, in ASMR, they often are), a giving up of control is involved in the video clip enabled by the remediation of haircutting. This is happening on various levels: The viewpoint of the video watcher is steered in different, sometimes potentially undesirable directions (e.g. the ceiling), while the performer withdraws from the range of vision. In addition, the viewer has to fill in the gaps in the image, not only because Heather Feather is only seen in close-up sections, but also because her visual presence is at times replaced by her voice and multiple auditory stimuli. As Marks concludes:

haptic images have the effect of overwhelming vision and spilling into other sense perceptions. This is in part because they do not provide enough visual information on their own to allow the viewer to apprehend the object, thus making the viewer more dependent on sound and other sense perceptions. This shift of sensory focus may be physical, or, as in film, through associating the available audiovisual information with other, remembered sense experiences. Film cannot stimulate the precise memories associated with a smell: only the presence of the smell itself can call them up. Yet a haptic image asks memory to draw on other associations by refusing the visual plenitude of the optical image. In addition, because haptic images locate vi- 
sion in the body, they make vision behave more like a contact sense, such as touch or smell (Marks 2002, 133).

ASMRtist Whispers Red - "Sleep Whisperer, Whisperer - Content Creator on YouTube" 110 proposes in an interview that ASMR videos featuring roleplays of intimate treatments are popular with users because they re-enact situations in which viewers experienced 'the tingles' or other comforting sensations in non-digital ('real life') situations, perhaps during childhood. In this sense, the haptic images and sounds become an amalgamation of overspilling sense perceptions that draw on memories. As Marks explains, a haptic image forces the memory to recall other associations when the visual pleasure of the image is neglected. In the case of ASMR roleplays, it seems that these are coming together: both physical and remembered sense experiences.

I also understand the images of Heather Feather's video clip to be haptic images, in the sense that there is a distorted picture of her. The video features fragmented close-ups, until she removes herself from the screen completely but stays audible, so that the viewer cannot apprehend the object but has to surrender to it. This haptic image "asks memory to draw on other associations" (Marks 2002, 133); in this case, the experience of being in a hairdresser's chair and getting a haircut.

Another aspect of haptic images is that they invite shared embodiment which blocks mastery over the other and instead requires a "loss of self in the presence of the other" and a "giving-over to the other" (2002, 20). This element, according to Marks, introduces an erotic element into haptic visuality:

What is erotic about haptic visuality, then, may be described as respect for otherness, and concomitant loss of self in the presence of the other. [...] A visual erotics that offers its object to the viewer but only on the condition that its unknowability remain intact, and that the viewer, in coming closer, give up his or her own mastery (Marks

${ }^{110} \mathrm{http} / /$ whispersredasmr.tumblr.com/, last accessed 10/12/2015. 
2002, 20).

What Marks describes as the erotics of haptic visuality might very well be the reason why many ASMR fans talk of 'braingasms': the erotic moment lies in the zooming in on and oscillating between different sensory perceptions and the accompanying loss of a monolithic perception of self.

To conclude, what I am suggesting here is that ASMR as a provider of visual pleasure (from brain tingles to feelings of relaxation or other intimate reactions) is best understood within the framework of haptic visuality. Intimacy conferred through ASMR videos, and the tingles experienced by some of their users, can be seen as resulting from the fact that haptic images "invite a multisensory, intimate, and embodied perception, even when the perceptions to which they appeal are vision and hearing alone" (Marks 2002, 133).

In this sense, ASMR is a digitally intimate genre that does digital media differently. Its haptic images, in combination with intimate storytelling and filmmaking techniques, extend from more conventional depictions of intimacy as 'romantic intimacy' or 'intimate treatments' to rather unconventional ones, such as 'repetitive relaxation' (the watching of/listening to monotonous activities or sounds). Following the framework of haptic visibility, this combination leads to upwelling memories, overspilling sense perceptions, and experiences of shared embodiment. In this way, digitally intimate viewing practices such as the ASMR trend differ significantly from normal viewing conventions or, as Wibke Straube argued in a different context, the viewer becomes an "entrant" into the scenery (Straube 2014, 63). In this way, the viewing experience can be seen as different in the sense that it is a more ethical, compassionate form of 'viewing' than, for example, the employment of an objectifying 'gaze', since here, as Marks argued, "the viewer, in coming closer, give up his or her own mastery" (Marks 2002, 20).

Having said this, I am also aware that ASMR women are nevertheless often portrayed as solely objectified. In the following section, therefore, I analyze how this objectifying perspective is explained and 
perpetuated, and how some ASMRtists break with this categorization, which has been imposed on them from the outside.

\subsection{Twisting Representations in ASMR}

In news reportage, ASMRtists are often represented and discussed in a sexualized and gendered manner. For instance, in a brief interview with the broadcasting station ABC15 Arizona, ASMR video starlet Jennifer, aka AppreciateASMR, is asked bluntly whether the fascination with her videos is not predominantly a sexual one (Hockridge 2013). This occurs against the background that one of Jennifer's videos has been flagged as inappropriate for an audience younger than 18 years by YouTube. ${ }^{111}$ "It's not intended to elicit a sensual or sexual response. That's not the purpose of these videos," Jennifer states firmly (ibid.).

The video in question is called _ $\sim$ Close-Up ASMR Caring for Sleep Mini $1 \sim \sim^{112}$. In the very short clip, Jennifer can be seen lying, presumably in her bed, saying that she is ready for sleep and that the viewer should relax and become sleepy as well. The camera perspective shows the upper part of her body, leaning towards the camera. More precisely, it shows her face, long blonde hair, the beginning of a cleavage. She is coming steadily closer with her lips while talking, reaching out to the camera's microphone - to create a binaural sound effect. The lighting in this clip is dimmed, with the result that the images are very grainy. This creates a low-quality image effect, which leaves Jennifer a little bit 'in the shadow'. It is not difficult to see why one would consider this video to be following particular conventions of sexual imagery.

In news reportage, ASMR women are sometimes compared to mystical or mythological figures like 'sirens', or 'unicorn girlfriends' (as in 'too good to be true', Graff 2013). In this way, cultural imaginaries are evoked that put them in particular boxes and promote traditional

${ }^{111}$ Accessed through my European internet connection, there is no sign of such a flagging.

112http://www.youtube.com/watch?v=6asPFYyzOHU\&list=UU4qFW_9Yn5 kzLdzD1Gi1b9Q, last accessed 10/03/2014. 
representations of femininity as tender, caring, motherly, sensuous, sweet, lustful, and so on. These perceptions are often encouraged by the performers because being tender, calm, pleasing, and so on, are crucial parts of the ASMR phenomenon, since many of these aspects trigger tickles in the users.

This is also reflected in the choice of adjectives and subjects that the performers use when choosing a descriptive performer name. Ethereal and feminine words like whisper, feather, gentle, rose, lilium, unicorn, ocean, breeze, and water are typical here. Nonetheless, calling them sirens is foregrounding the sonic dimensions of the video clips (the seductive whispering, affective voices, and sounds are stressed) and connecting them to some kind of subliminal threat at the same time. In Greek mythology, sirens would distract sailors until they lost control of their steering or were wrecked upon the coastline (as, for example, in Homer's Odysseus or the German folklore myth around the Lorelei). ${ }^{113}$ The viewer or listener is lured into danger or even to their death. Relating ASMR videos to sirens associates them with a threat, a threat that involves 'letting oneself go' and losing oneself in the hands of someone of whom it is not clear whether she means well. There is an anticipation about whether and when she will give one the tingles, or lure one into sleep. In the context of sirens, the voice becomes a sexualized weapon.

${ }_{113}$ In Heinrich Heine's famous poem based on the folklore, heartbroken Lorelei is sitting on the eponymous rock, singing and combing her hair: "She holds a golden comb/Singing along as well/An enthralling/And spellbinding melody" (translation from http://german.about.com/od/literature/a/Heinrich-Heine.htm, last accessed 1/12/2015). Interestingly, hair combing has its own sub-category of popular ASMR videos because many viewers experience the feeling of having their hair combed by another person when they watch these clips, which they find pleasurable. In this sense, Heine combined two mesmerizing elements in The Lorelei, the seductive voice and hair combing, which in the myth distract the seaman to such a degree that he forgets about the dangerous rocks and is devoured by the waves. In a way, a mystical female figure was being blamed for unfavorable seafaring conditions (woman as part of 'nature') or poor sailing skills, i.e. the sailor's failure and, in a similar way, the ASMR videos may be being judged when the performers are called sirens. 
I argue that the reportage describing ASMRtists as sirens, unicorns, and so on attests to an outsider perspective that sees these ASMR videos merely within a psychosexual context. It does not take into account the diversity of the performers or viewers, an accusation that was also made against feminist film theory surrounding the gaze (Smelik 1995). It also does not take into account how women take control of the gaze/camera and reflect it back onto the viewer, how they look at the viewer, or how they let the viewer see through the camera while it is under their control (rather than women being only looked at by the camera).

The point is, as I have mentioned earlier, that in Mulvey's conceptualization, the gaze is actually a triple gaze of camera, narrative, and editing that turns the woman into a spectacle (Mulvey 1989). In this sense, the perception of ASMR performers as sirens turns them into spectacles and mystifies their agency in terms of camera work, narrative, and editing. A similar point has been made about the historical predecessor of the whispering women, i.e. the camgirl. In the following, I will make a short comparison.

\section{ASMRcamgirls}

Similarly to the sexualization of ASMR videos, there has been the sexualization of camgirls, who in some ways can be understood as forerunners of the women in ASMR videos. In her analysis, Michele White argues that

[w]ebcams certainly have the potential to reinforce gender norms and provide erotic views. However, webcams also offer women a heightened level of control over their representations. This argument is different than the kind of feminist analysis that presumes that visual media technologies are always part of an objectifying process that permits viewers to look upon and possess women's bodies. (White 2003, 9) 
White does not deny the dimension of camgirls as a submissive, pornographic, or semi-pornographic genre, which is highlighted in the name camgirls:

The cam girl label suggests to spectators that women will respond to monetary rewards with personal favors, always be visibly available on the webcam, able to chat and respond to email on demand, recognize individuals by waving or holding up their name to the camera, smile and look happy while they are depicted, offer nude pictures or other salacious material in their archives, and flash their breasts or reveal other parts of their bodies on the webcam. (White 2003, 15)

Similarly to Marks' findings (but without the notion of haptic images), White argues that camgirls are "too close to see" $(2003,7)$ and thus render the notion of only being looked at more complicated. Also, they ultimately decide what there is to see, as I have also suggested in in the case of Heather Feather's hairdressing video.

Additionally, the notion of the camgirl as someone solely to be looked at becomes even more complicated when the 'original' camgirl, credited as Jennifer Ringley, is considered. Although today it almost seems mundane, 20 years ago it almost caused a moral panic when she, as a 19 year old, decided to connect a camera to her computer, which would send a picture from her student room out into the digital world every 15 minutes. JenniCam was born and became the ultimate expression of digitally broadcast mass-mediated intimacy. For seven years and eight months, four times an hour (or every three minutes for subscribers), she sent a picture of whatever she was doing in her student dorm room out into the cyberspace 'void' - including pictures of her staring dully into space, holding up handwritten messages like 'I feel so lonely', and masturbating.

Ringley stated that she did not want to impose a filter, but to show her life in a straightforward and immediate way. Therefore, with just a few exceptions at the beginning, the camera was never turned off. Sexuality happened, but it was not central. At the peak of her popularity, 
JenniCam had three to four million daily viewers, was featured on the front pages of magazines, and was welcomed on famous talk shows such as David Letterman's.

In this way, Ringley invented life casting, which had not previously been a genre of the digital era, and in contrast to what today is often understood as camgirl (the porn dimension), JenniCam is considered by many to be conceptual art, and by some feminist writers to be a cyborg subjectivity. Krissi M. Jimroglou, for example, has argued that JenniCam

exists as a cyborg, disrupting boundaries and resisting monolithic definitions of the subject simply by the fact that Jenni has integrated herself with technology. Just like Haraway's cyborg, JenniCAM embraces aggregation rather than unification. In confusing dichotomous boundaries such as body/machine, private/public, and real/fiction, JenniCAM forces a rethinking of the female subject in relation to each binary opposition. $(1999,442)$

In line with my own argument about the agency of female ASMR performers, White and Jimrogulu transgress the notion of the gaze in relation to camgirls and add a degree of cyborg subjectivity that confuses dichotomous boundaries. In the case of ASMR, I would like to add the transgression of the digital/non-digital boundary since the performers regularly try to reach through the screen and give the viewer the feeling of being touched, which in turn is often experienced by the viewers as a tingling sensation. This is also achieved through the DIY film-making techniques used by the ASMRtists.

In these film-making techniques, the same woman is both in front of the camera and behind it, she is both composer and composed. I would suggest that ASMR videos, with their DIY approaches to creating intimate digital places and relations, can be seen as forerunners of a 
new genre that is also redefining intimacy as remediated. ${ }^{114}$ Some ASMRtists are taking their experience with immersive, intimate digital experiences into a more professional realm and have started virtualreality production companies (Abbruzzese 2015, n.p.).

Alongside these technological ways to twist the male gaze, I will show in the following section how the normative gaze is also subverted through strategies of irony within some performances.

\section{Intimate Complicity}

In Heather Feather's performance of being 'science girl', which lasts for more than an hour, I see her sitting across from me at her lab desk. She is wearing transparent goggles and her lab coat; behind her I see the outlines of a white sink module. Otherwise, the background seems clean and neutral, and there are not many details upon which to linger. After introducing herself, CalmingBreeze slowly and quietly checks my brain for parasitic worms (with what looks like a flashlight). I pass this test, but there are more to come: She puts "little sticky sensors" onto my chest and connects me to a machine that is out of my sight. She is calm and careful, asking me if I feel comfortable, and checks my heartbeat with the help of her headset (my heartbeat is nice and steady, she says). I now learn that I am her new intern and this is why she is running these tests on me. This is also why she is testing my love of science in the next step (by putting a love thermometer into my hand, a glass vessel with colored alcohol in it, that 'boils' quickly at body temperature). She demonstrates it herself, stating "I love science. I am not afraid to prove it." For the next hour or so, CalmingBreeze focuses on her work and does what scientists/chemists/lab workers might do. She scribbles notes and makes calculations with the help of

114 Interestingly, Laura Marks argues that "much of the video work that first experimented with haptic qualities was made by women, often by feminist or lesbian makers interested in exploring a different way to represent desire" (Marks 2002, 16). 
her calculator. Her early attempts at small talk ("You've had lunch already?”) get less and less, because she is so focused on her work. Often it seems as though she is talking more to herself than to me, her intern (“5 ...7...”). She seems like a very determined researcher (taking notes with a serious facial expression and complaining about the amount of paperwork she has to do, sighing when one of her chemical tests does not produce the right results). I see her mixing diverse chemicals in Erlenmeyer flasks and test tubes, heating them over a Bunsen burner, all while creating many repetitive and tender sounds: The scribbling on paper, the clinking of Erlenmeyer flasks against glass funnels. So far, this clip is following the unofficial script for an ASMR video with many likes: A pretty girl, soft whispering, repetitive and comforting sounds, and close personal attention.

But towards the very end of the video, very surprisingly and unexpectedly, there is a turn of events that possibly most viewers miss because they have already fallen asleep. She takes out a little sachet filled with diamonds, and out of the blue asks me to smuggle them to Harvey Dent Plaza. Without losing her softness and calm, she casually explains to me that the liquid she has created is actually toxic; that she will put the liquid and the diamonds in a perfume bottle to disguise them; and that I will get one of the smaller diamonds as a reward later on, if I succeed in my task.

The clue to what is happening here lies in her casual mentioning of Harvey Dent. Harvey Dent is the name of a comic-book character in the Batman series before he turns into super-villain Twoface. ${ }^{115}$ CalmingBreeze is thus evoking a Gotham City-style outside world. Her whole performance of 'science girl' creates a hyperreal world, where she is creating images of a scientist/lab worker/chemist/diamond smuggler by using and blending different accessories and conventions into a wild mix.

115 In the Batman saga, Harvey Dent was a loyal and cherished politician of Gotham City. But after half of his face is disfigured after an acid attack, he becomes the insane villain Twoface. According to writer Bob Kane, the character was based on Robert Louis Stevenson's Strange Case of Dr Jekyll and Mr Hyde and shows signs of schizophrenia. 
As Jean Baudrillard famously argued, a cultural shift has occurred in which the relationship between reality and image has changed from one of image/reflection to one of image/simulation (Baudrillard 1994). While the simulacrum is a false copy of something, in his take on media effects and culture in the postmodern world of TV and modern media, Baudrillard saw the idea of the true or false copy destroyed: all that is left is simulations of reality, which are no more or less real than the reality they simulate. 'The third order of simulacra' marks our present age, which is dominated by simulations, things that have no original or prototype. For Baudrillard, it is cybernetic machines, computers, and virtual reality $(1994,84)$ that belong to this new world order, but also popular news and DNA. The image, in this current time, bears no relation to reality at all but appears 'hyperreal' - that is more real than the real - a sign or representation without an original referent.

In the same way, there is no original to CalmingBreeze's actions. I understand her mentioning of Harvey Dent as an acknowledgment and as playing with that insight: she is pointing out the hyperreality of her performance and having some fun with it. This is why she introduces this plot twist at the very end of the clip, when most of her viewers are probably asleep - as though it was a little joke she performed mainly for herself.

At the same time, this ironic twist breaks with the nice girl image. Even though CalmingBreeze stays in character throughout the whole clip, she is also showing that she is twofaced: She is not only a nice girl, but a precise chemist and smart criminal as well. She is introducing notions of complicity and hierarchy into her performance. I am her intern, and at the same time I am becoming complicit in her criminal activities. In this way, elements of losing oneself by coming closer and giving up one's mastery are invited by the narrative as well. In other words, I suggest that, in addition to haptic images and particular directorial tricks (such as steering the gaze with camera angles), humorous subversion is another tool that makes the ASMR experience an example of doing new media differently, here in the sense that it can break with viewing conventions and expectations of female representation. 


\subsection{ASMR and the Science of Sleep}

In this section, I am 'zooming out' my perspective on ASMR to focus on a broader level of discussion instead of analyzing particular video clips. The layer of the ASMR phenomenon that I am interested in here is that the videos are considered by many to have a therapeutic effect. Comments such as:

I'm not sure if ASMR works on me or not, but I enjoy this video because you perfectly understand those who are stressed out and anxious. I always feel better after watching this for some reason. I always find myself coming back to this when I am depressed and it helps me. Thank you so much for this video : $)^{116}$

Or

your video just calmed me down big time thank you so much ! I was having trouble sleeping due to acid reflix that i have been dealing with asmr puts me to sleep during my worst times. :) thanks so much. ${ }^{117}$

are common in the comments sections of the corresponding ASMR videos. While the claim that ASMR can cure sleep problems and a range of ills such as depression, concentration disorders, anxiety, or migraines is strongly promoted within the ASMR community (Abbruzzese 2015), there is little clinical proof of the healing effect of these videos.

While digital cultures around ASMR used to be a petri dish for theories of how ASMR works on the brain and how it can be beneficial for one's well-being, a recent quantitative psychological study by Emma L.

$116 \mathrm{https}$ // www.youtube.com/watch?v=wo8YaVspigQ, last accessed 17/09/2016.

117 https://www.youtube.com/watch?v=vMcqVGrQxZs, last accessed 17/09/2016. 
Barratt and Nick J. Davis (2015) tentatively confirms some of the theories: That there is an overlap with synesthesia ${ }^{118}$ for relatively many people; that the well-being induced through ASMR videos can indeed reduce symptoms of depression and chronic pain for some; and that ASMR can induce a "a flow-like mental state" similar to what Mihaly Csikszentmihalyi has called flow in his popular scientific publication Flow: The Psychology of Optimal Experience (2008). Flow in his account means a state of immersive bliss through (creatively) satisfying work or activity. The idea put forward in Barratt and Davis' study is that this state or flow might also be induced by watching other people perform activities with the help of mirror neurons. ${ }^{119}$

Even though ASMR has now also been investigated from neurological perspectives, it has been (and often still is) considered a phenomenon that is not to be taken seriously and is rather a quirky socialmedia trend. This implies that people come across the videos on YouTube or other social networks, find them interesting or fashionable, and want to become part of the sensations and the ASMR community,

${ }^{118}$ Someone with synesthesia might be able to experience such things as a number or letter via additional cognitive or sensory pathways, perhaps by experiencing digits or sounds in particular colors, a condition that is rare and whose existence was contested for a long time.

119 Mirror neurons are a group of neurons located in the frontal lobes of the brain. They are a subset of command neurons, which react when we perform different commands or actions. What is special about the mirror neurons is that they also react when we observe someone else performing the same action. Mirror neurons were discovered in the early 1990s (Rizzolatti and Fabbri-Destro 2010), and as a relatively young discovery their function is subject to much debate. Some researchers argue that they are important for imitation and the emulation of other people's actions, and have played an important role in learning and passing on skills. Other mirror neurons respond to touch. In other words, a subset of sensory neurons fire in the brain when people watch other people being touched (cf. Ramachandran and Altschuler 2009, 1703). For some people, mirror touch can give a tactile sensation on their own body, as if they were being touched themselves. This is referred to as mirror touch synesthesia (linked to "heightened empathic ability", Banissy and Ward 2007 and Ward 2007). 
thereby creating ASMRers along the way, instead of experiencing an actual physiological response (Novella 2012).120

Lisa Blackman has pointed out that a reorganizing of the ontology of subjectivity and corporality is currently taking place within cultural theory, which focuses on "processes, practices, sensations and affects that move through bodies in ways that are difficult to see, understand and investigate", a focus which touches on the idea of the solidity and insularity of the subject (Blackman 2012, IX). Therefore, she observes, concepts such as speed, mobility, immateriality, fluidity, multiplicity, and flows are (re-)examined to understand how intimate attachments or affective relations emerge.

I would like to suggest that the resistance to taking ASMR seriously could be based, firstly, on the fact that ASMR and its multitude of different symptoms, affects, and flows which move through the body are difficult to see, understand, and investigate, as Blackman explained in relation to affective bodies. At the same time, the fact that the body becomes an affective body touches on the idea of the solidity and insularity of the subject, which might be an unpopular vantage point.

Secondly, it is a fact that ASMR is a phenomenon that became visible through digital media and networked online communication, and this undermines its plausibility for some. As I have quoted from Meßmer, Schmidbaur, and Villa earlier, there is a tendency to want to see intimacy as something unmediated, which can be called a romantic but digitally dualist assumption.

Through the mediation and remediation of ASMR, people are able to express unique, personal experiences on a large scale, finding like-

120 This might also be the case because one narrative of the origin of mediarelated ASMR is the story of many people simply googling 'that weird feeling' and in this way finding entrance into the online community. Karissa Ann Burgess of asmr-research.org reports: "I have a vivid memory of being in a packed elementary school gymnasium, and a teacher was reading 'The Cat in the Hat' aloud to all of us. I remember this euphoric feeling in my head that I never wanted to end. I experienced this wonderful feeling, which I actually referred to as 'THE FEELING' at the time, frequently when being read to as a child. [...] I have been fascinated with this group of individuals ever since I decided to Google [sic] 'the weird feeling' one fateful day [...]" (Available online at http://kburgess.asmr-research.org/, checked on 3/8/2014). 
minded people, connecting with each other, bringing the topic into public awareness, and making it a research interest. Still, the question of the chicken and the egg remains: What was there first, a 'hardwired' receptivity to ASMR stimuli in some people or the 'contagious' awareness of such a thing via social media?

In his book on current mediatization processes and their impact on society, 24/7: Late Capitalism and the Ends of Sleep, Jonathan Crary draws a dark picture of how the era of what he calls late capitalism is attacking the last arena not completely taken up by money, work, and consumption: sleep. Drawing on examples as different as the search for performance-enhancing and sleep-reducing drugs in military medicine and the design of benches in public places such as train stations and airports that prevent people from ever finding a sleeping position (2014, 26), he investigates how temporal frameworks have shifted to adjust human routines to the demands of the market: "Time for human rest and regeneration is now simply too expensive to be structurally possible" $(2014,14)$.

He argues that this becomes especially visible in the context of digital media. He does not buy into the hype that such media could lead to drastic social transformations (as was often suggested, for example, in the case of the Arab Spring), but rather sees the fact that it is possible to surf, go online, watch, read, shop, and communicate $24 / 7$ as another attempt of consumer society to commodify human time. Sleep, in this context, becomes a good that needs to be defended and protected by society "as the most private, most vulnerable state common to all" $(2014,25)$.

From this perspective, it is interesting that ASMR videos are considered by some to be a kind of self-help for sleep problems and other psychological issues. This logic follows neoliberal ideas of self-improvement and responsibility; it is implied that one only needs to turn on the computer to be cured. While Berlant talked about the mass spreading of intimacy into talk shows and self-help literature (1998), Garde-Hansen and Gorton argue that "online culture is characterized as both a therapeutic DIY toolbox and a folk devil - the cause and cure for all social ills in one indistinguishable stroke" (Garde-Hansen and 
Gorton 2013, 49). Similarly, it could be said that ideas of intimacy and the intimacy of sleep are sold as cures in "a therapeutic DIY toolbox", which makes sensations and intimacy calculable, accountable, and consumable. This puts ASMRtists into the position of affective laborers. As Michael Hard explains:

Health services, for example, rely centrally on caring and affective labor, and the entertainment industry and the various culture industries are likewise focused on the creation and manipulation of affects. (Hardt 1999, 96)

From a position of seeing digital media as an attempt to commodify time, I would like to suggest that the work of ASMRtists also has to be considered as affective labor, while the watching of the videos as a kind of self-cure can be seen as an attempt to substitute for missing care work, whether it be private or medical. Following Crary's perception of sleep as a last bastion of the personal and the private, ASMR as a therapeutic sleeping aid extends the range of intimacy online: another intimate, private realm potentially becomes remediated into a digital commodity.

\subsection{Discussion: A new Genre of Intimacy?}

ASMR is an idiosyncratic example of current everyday internet practices that strikingly illustrates the diverse forms that digital intimacy can take today. However, there is a danger of putting the women of ASMR into a particular box, as happened earlier to the camgirl. This happens, for example, through the comparison with mystical creatures like the siren, who can use her voice or hair combing as a means to electrify men and bring misfortune to them. Seeing female ASMRtists (or cam girls) through a psychosexual lens alongside the vocabulary of, for example, fetishization and voyeurism (cf. Hudelson 2012)is only one side of the coin. This way of representing them ignores the fact that the performers are not only in front of the camera, but also behind it, and treats the viewers as one homogeneous group. 
However, there are several angles from which watching/experiencing ASMR videos means to do digital media 'differently'. For example, as Laura Marks has explained, haptic images (for instance, grainy close-ups that are 'too close to see' from an objectivizing outside) invite an intimate, multisensory, and embodied perception that complicates the logic of the gaze. What also challenges this logic is the fact that the whispering women are defining and developing a new genre of intimate, affective, and somatic videos by controlling the narrative, direction, and editing of the clips.

Yet another way of twisting representation happens when the performers work with ironic twists and hyperbole, or create hyperreal worlds. The example of 'science girl' discussed above, for example, does so by mocking the conventions of science and turning cold, clinical lab practice into an intimate, sensual experience.

Conversely, it has to be noted that from a more critical perspective, ASMR as part of modern digital media remains within the logic of neoliberalism. Just as Crary has argued that previously untouched domains such as sleep are becoming commodified, it could be said that ASMRtists' performances of intimacy become affective labor, or that they are taking care of a digitalized society with changing intimate needs, thereby creating a more affirmative digital culture for some. 

CONCLUSION:

\section{COUNTERING}

DIGITAL INIMICALITIES 



\section{Conclusion: Countering Digital Inimicalities}

To rethink intimacy is to appraise how we have been and how we live and how we might imagine lives that make more sense than the ones so many are living. (Berlant 2000, 6)

In my theory chapter (chapter two), I discussed the fraternal kiss between Honecker and Brezhnev to illustrate the complexity and transformability of the term intimacy. My intention was to illustrate the possible dimensions of how a single instance of intimacy can travel and transform, how this kiss was remediated, remixed, materialized, commercialized, politicized, and thus ultimately became more than the sum of its parts. The point of departure for this dissertation was that intimate affectspheres are being transformed at accelerating speed, and in many diversifying forms, in the context of digitalization. Following this line of thought, my main research questions were concerned with how digitally intimate initiatives and approaches may confront and counteract inimical media cultures, and how digital intimacy can be conceptualized as a tool for feminist internet politics.

Although kissing is not necessarily a crucial element of ASMR videos, in this particular subculture it is easy to see how intimacy is transformed in a digital context, to the extent that the boundaries between digital and non-digital intimacy start to blur (e.g. when sensory pathways cross and people feel as though they have been physically touched). The inimicality in the case of ASMR, on the other hand, was a question of representation and objectification, which in this particular setting means acquired viewing habits which suggest that the 'whispering women' are mainly objects for a 2.0 version of the (straight, male) gaze. However, I have suggested being critical of this understanding. I drew a comparison with camgirls, and more specifically the pioneering figure Jennifer Ringley, and I argued that in such cases 
women sometimes gain a heightened level of control over their representation, a fact that challenges the idea that visual media technologies solely objectify women.

I argue that this happens, firstly, because viewing in this context has to be seen in Laura Marks' framework of "haptic visuality", in which close, haptic images "invite a multisensory, intimate, and embodied perception" (Marks 2002, 133) of the material, which ultimately leads to a letting go of visual control, and of one's sense of separateness from the image $(2002,13)$. Secondly, this heightened level of control is also achieved through the narrative strategies of some ASMRtists, such as their steering of the gaze, ironic subversion, and cinematic tricks (which I also describe as creating an 'intimate complicity' towards the end of chapter six). Altogether, these strategies affectively confuse dichotomous attributions such as online/offline, real/fiction, private/public, body/machine, and close/far, and disrupt the sovereignty of seeing as a superior sense, therefore inviting a rethinking of the subject in relation to these dichotomies.

As a novel phenomenon of producing and using intimate stimuli on such a large scale (considering the millions of clicks and viewers), ASMR is doing digital media differently because it breaks with established patterns of visual pleasure, and thereby creates intimate affectspheres that can re-define intimacy beyond more conventional understandings, such as necessarily related to kinship, sexuality, or physical proximity. This reconfiguration of intimacy could thus be considered a form of counteracting established forms of representation, and as such providing a generative element: new ways of looking and engaging with the other lead to an experience of shared embodiment. This is an important counter position, a potentially more ethical way of doing digital media and its visual cultures, because it finds alternatives to solely 'gazing at' and 'being looked at', and its accompanying power dimensions.

If ASMR is provoking new ways of interacting with digital visual cultures, women*-centered coding networks like HackerWomen are seeking to change another digital culture and its sociotechnical networks, insofar as they address the situation of too few women working 
in coding and computing, a situation that historically has not always been the case. As I have shown, their strategy is to try to change a cultural parameter, a parameter that has discursively connected notions of white, straight masculinity with those of detachment and rationality, and those in turn with successful computing (Ensmenger 2010; Kendall 2011), by encouraging more women into tech.

What I have criticized here is their appellation of women as such. The re-branding of coding as a social, fun, and passionate activity is certainly working on one level to do digital media differently, in the sense that it counteracts the cultural idea of coding as lonely and isolated. However, there is also the pitfall that it could reinforce the idea that women primarily bring 'soft skills' to the table. This might position them in opposition to the stereotype of a more rational, nerdy, and detached male programmer, and thereby potentially cement the gender binaries that these organizations originally wanted to erode.

I do find the coding initiatives to be a welcome and necessary approach to helping individuals and communities who want to learn coding. Nevertheless, the ability to counteract inimicalities (like persistent cultural restrictions in terms of unfriendly work climates and hostile conditions when it comes to women in computing), must be seen as compromised here from a feminist standpoint.

The emphasis on supposedly feminine traits and soft skills indirectly supports the cultural parameters as described above, which ultimately perpetuates a binary thinking: that the male nerd can rationally detach (his mind) from his surroundings, while women need emotional access in order to connect with the machine. In conclusion, initiatives such as these open up some space for female programming identities, but do little to challenge the existing gender binary. The question of how much the creation of a 'female programming' of this kind ultimately can counter online inimicalites as I have described them, and if an increase in female programmers would automatically do so, is uncertain. Digital media scholar Malin Sveningsson (2012), for example, has pointed out that in many tech domains, an increase in female par- 
ticipants provides for less hostile climates, but that the women as facilitators for this type of change often pay a high price (e.g. that they are reduced to this caring, facilitating role).

In this sense, my second empirical case study about women's coding initiatives is noticeably different from the other two, because it seems to be more of a reactive than a generative initiative. It is reacting to an existing problem but does not, from my point of view, generate convincingly novel approaches that take into account the fact that gender and technology co-shape each other (Wajcman 2010).

I therefore suggested that, instead of using emotionally charged notions of sociality, passion etc. (which are focused on human-to-human interaction and predetermined, normative emotions), it could be more useful strategically to engage a more open perception of intimacy, as digital intimacy. Digital intimacy, here, becomes an affective way to grasp other bodies (whether they be, in Deleuze's sense, digital, social, visceral, or otherwise, 1988), and to rethink intimacy with the digital in terms of more mobile attachments (Berlant 1998, 284), as an "affective moving back and forth between the analog and the digital" (Sundén 2015a, 140).

In this suggested take, the counteracting and generative component could be a shift in perspective in which intimacy with the digital is not related to particular emotionally charged assets, but would open up paths to understanding coding and its environments as embodied, physical, affective encounters without running the risk of essentializing particular positions within it. Put simply, I am imagining coding initiatives that allow the technology to shape bodies and bodies to shape technologies, instead of frameworks within which both positions are rather static, even though it might be unclear how such an initiative might look like in detail.

Lastly, the anti-trolling platform, hatr, offers a novel way to understand and work with a severe digital inimicality, which has been much discussed recently, namely hate speech and online harassment. With its strategy of grabbing and displacing hateful comments, hatr manages to make visible something as difficult to capture as hate, testifies to its affectivity, and protects the addressee. 
I have demonstrated how this strategy of detaching hate from the addressee disrupts an affective economy of hate (Ahmed 2004) and creates a digital testimony. In this way, the severity of such online hate is documented and becomes comprehensible for the empathic reader of hatr.org. The hater, the sender of the hurtful message, on the other hand, is displaced and made "open to an unknown future" (Butler 1997, 4).

The counteracting element here is the most straightforward one, in comparison to the previous cases: It is a novel attempt to place something in opposition to the epidemic of hateful and hurtful online communications that particularly female, feminist, or LGBTQI activists have increasingly reported over the last few years. The generative aspect is thus the creation of a digital, affective testimony that concretely protects the activist blogger, confuses the hater, and interrupts the hateful economy. Significantly, it retains its affective charge and in this way becomes a testimonial with mobile attachments (in the sense that it becomes much more unpredictable which of the readers of hatr.org will be affected, and in what ways).

In my search for novel and creative ways to counteract inimical digital communication cultures, in this thesis I have presented and analyzed three different initiatives that do so, albeit in very different ways and with varying degrees of success. However, I am not interested in success as such, ${ }^{121}$ but in the ways in which these initiatives and their strategies can be conceptualized as tools for a feminist internet politics. I will lay out these ways in the following section.

\footnotetext{
${ }^{121}$ I am following Jack Halberstam's assessment that success as a notion tends to uphold particular societal standards, which often carry (hetero-)normative undertones. In his Queer Art of Failure (2011), conversely, failure is re-evaluated as holding queer, creative potentialities that counteract neoliberal conformity and individualism. In this sense, the 'success' of ASMR, hatr, or the women's coding initiatives should not be measured in terms of revenue, marketability, or recruiting numbers, but rather as analytical opportunities for societal change in the arena of digital communications.
} 


\section{Remixing Digital Intimacies for a Feminist Internet Politics}

The tools that can be abstracted from my three strategic examples are thus centered around creative play with language and its affective economies; around the developing of forms of agency in burgeoning new digital genres that invite different forms of interaction and shared embodiment; and around the opening up of an understanding of computing as an affective oscillation between the analog and the digital.

Central to all of these discussions is a way of conceptualizing digital intimacy as a remediated form of intimacy. Digital intimacy within my three examples is, in my understanding, juxtaposed with more normative ideas of intimacy, which in turn feed into some aspects of digital cultures and resemble the digital dualist assumption that what is happening online or in the realm of the digital is somehow not 'real' or does not 'matter'. For example, the idea that intimate affronts (e.g. hate speech) are not 'real', and as such do not have an actual effect on bodies when they occur online, mirrors the fallacy that intimacy can only be 'real' when happening in conventional forms (i.e. non-digitally and/or face-to-face). Cultural parameters around coding, furthermore, are built on the false assumption that there are 'right' and 'wrong' forms of attachment or detachment vis-à-vis the digital. And in ASMR, the 'whispering women' at times challenge viewership as an objectifying distance within digital visual cultures and thus the idea of intimacy as non-mediated.

In other words, my conceptualization of digital intimacy is opposed to these perceptions. In this thesis, I consider hate speech to be highly affective and as such embodied, I argue for attachments to the digital to be seen as a much more mobile processes, and I seek to disentangle ASMR from the idea that it is something to merely look at, but instead argue that it transforms viewership affectively. 
Thus, digital intimacy as I have discussed it is not about tender loving care (TLC ${ }^{122}$ ). Digital intimacy does not boil down to being 'sweet' in an ASMR video, or providing a 'social' component in a women*-centered coding course, or treating hateful words as if they 'don't matter'. Rather, it is about moving along with affective flows, paying close attention to the co-shaping of novel technologies with oneself, the body, and subject positions, and critically interrogating the assumptions and norms on which many of the contemporary engagements with the digital are built.

\section{Remediating Older Forms of Feminist Activism}

One additional finding of my research that I had not anticipated is the extent to which older, established strategies and tools of feminist thought and activism have been remediated and reintroduced into digital contexts. Many of the initiatives that I have presented aim to create safe spaces for women and other marginalized groups, and work with the notion of consciousness raising, and/or catalogue sexism and other forms of discrimination.

Hatr.org, for example, remediates all of these activist modes: Its strategy of displacing hateful comments creates safe spaces for bloggers and writers, protects discussions within the comments section from crass interruptions, and a trigger warning makes sure that users know what to expect upon entering the site. Consciousness raising and cataloguing sexism remain crucial forms of activism in a digital context, because they make patterns and structures of subordination visible (Ahmed 2004, 172).

Similarly, coding initiatives like HackerWomen strive to create safe spaces by promoting and encouraging social, collaborative, and fear-free coding setups. Just as feminist safe-space initiatives, they also

${ }^{122}$ This term is usually used to describe jobs that require a heightened level of empathy, such as nursing or raising children. 
often mention anti-harassment policies in their code of conduct (for example in Girl Develop It, Railsbridge, Railsgirls, Codebar). ${ }^{123}$

It could perhaps even be argued that ASMR follows a safe-space logic, albeit not in a political fashion. This is visible not only through the fact that the worlds created in some of the clips try to create happy places and a retreat from everyday life, but also through the fact that many ASMR enthusiasts, both on the producing as well as on the receiving end, see themselves as part of a community that supports each other and cares for each other: the ASMRtists through providing digitalized intimacy and the receivers through their fandom and gratitude expressed in comments, and the mutual exchange in different digital forums.

\section{Digital Intimacy and the Neoliberal Context - Money Can't Buy Me Love}

Another finding of this research is the degree to which the rules of the free market are influencing all my empirical material. This is not surprising as such, but I had not anticipated that a neoliberal market logic would become so dominantly visible in my material. As Wendy Brown has established, neoliberal settings have an impact on the organization of subjectivity, meaning that subjects become 'entrepreneurs': They have to work on themselves, are responsible for their own optimization, and have to make themselves attractive to an always-demanding (job)

\footnotetext{
${ }^{123}$ For example, on the Girls Develop It homepage it says: "Harassment includes offensive verbal comments related to gender, gender identity and expression, sexual orientation, disability, physical appearance, body size, race, or religion; sexual images in public spaces; deliberate intimidation; stalking; following; harassing photography or recording; sustained disruption of talks or other events; inappropriate physical contact; and unwelcome sexual attention. Adults who identify as men are welcome at our events, and it is expected that all participants be treated with equal respect. We do not tolerate harassment of Girl Develop It participants in any form. All communication should be appropriate for a professional audience. Participants asked to stop any harassing behavior are expected to comply immediately."

(https://www.girldevelopit.com/code-of-conduct, last accessed 13/10/2016).
} 
market (Brown 2003). This is something that affects women more often and more strategically than men (Scharff 2014, n.p.).

This neoliberal logic in my strategic examples comes in various shapes and forms: as a particular kind of individualistic 'Facebook feminism', in the sense that some of the coding initiatives can be seen as means to improve one's own position, rather than collectively fight for social change (Faludi 2013); as an emotional drain and loss of working hours through handling forms of online harassment (see Hess 2014, and also in the context of hatr); as affective forms of labor in ASMR (cf. Hardt 1999; Gregg 2011); and even as attempts to 'monetize the hate'.

In their framework of Awkward Politics (2014), Carrie Smith-Prei and Maria Stehle analyze different forms of contemporary feminist activism and art projects in a transnational context. ${ }^{124}$ Awkwardness has been re-appropriated by these scholars in order to talk about such initiatives and forms of activism while considering their sometimes "[j]oyful silliness and awkward inappropriateness" and insisting on political relevance at the same time, despite "their entanglement in a neoliberal culture industry that relies on cruelly optimistic relations" (Smith-Prei 2014, 83). Awkwardness for them thus becomes a nonnormative aesthetic and affective state, a theoretical device to account for uncertainties in twenty-first-century feminist activisms that is highly influenced by DIY aesthetics, digital and social media, and that do not mind, as they quote Haraway's famous dictum, "staying with the trouble" (quoted in: Smith-Prei 2014, 83). In this vein, it could also be argued that my three strategic examples, which are intimately entangled with neoliberal culture industries and businesses

are awkward not only because a neoliberal digital economy enables them but also because these activisms, which doggedly persist in their political actions in spite of this enabling, simultaneously reveal the false promises of neoliberal capitalism. They do not or cannot detach from the

124 Such as the work of performance artists Lady Bitch Ray, novelist Helene Hegemann, or diverse Pussy Riot (solidarity) actions. 
economy that enables them; rather, they stick to that economy in an uncomfortable, awkward manner. (Smith-Prei and Stehle 2016, 11)

This 'sticking awkwardly to an economy' becomes visible in my material, even though it is not labeled as feminist activism (apart from hatr.org, which communicates a queerfeminist situatedness). This awkwardness becomes visible, for example, in ASMR when the performances are considered from the perspective of affective work (Hardt 1999) and when the use of videos is framed as a form of self-help or care; that is, when health care becomes a personal issue that individuals have to manage for themselves.

In a similar way, the coding courses are centered around different forms of affective work. Not only do the organizers offer an additonal 'service with a smile' by trying to make the workshop or course experience more encouraging, passionate, social, and so on, but the participants' lives are also affected by the changing demands of their workspaces, which creep into their work/life balance and intimate lives (Gregg 2011).

A similar argument can be made about hatr.org. Digital activist work is affective work and draws on physical and emotional resources. In this way, it also becomes affective labor and is usually also free (unpaid) labor. In any case, it makes living and working online more exhausting, less profitable, and more time-consuming (Hess 2014).

Of the discussed initiatives, hatr is the only one to "simultaneously reveal the false promises of neoliberal capitalism" (Smith-Prei and Stehle 2016, 11). In a further tongue-in-cheek twist, the hate is transformed or translated into hard cash (this happens indirectly through the sale of advertising space on hatr.org). This is an indication of how hate, even in the abstract, is not immaterial and, just like digital money, it has an impact on (affective) economies. 


\section{Embracing Digital Intimacy}

Donovan O. Schaefer, author of Religious Affects (2015), describes a common goal of affect theory as exploring "the possibility of sliding together analytical tools used to pick apart both highly individuated and highly social contact zones - bodies and histories - as incarnated realities" (Schaefer 2015, n.p.). In this fashion, this doctoral dissertation has investigated such incarnated realities in the sometimes elusive sphere of the everyday cultures of digital media. Therefore, I have, as Schaefer describes it, combined (or remixed) highly social and highly individual affective contact zones, in order to generate situated snapshots of what it means to be digitally intimate today.

Furthermore, from the perspective of feminist cultural studies of digital media, I have focused on the frictions and stumbling blocks that mark these digital contact zones today, and discussed them as digital inimicalities. Lastly, in the spirit of remix - which aims to take existing elements and combine them in a way that lets them each appear in a new light while also creating an entirely new object - I have extracted particular tools for a feminist internet politics from my three strategic examples.

The theoretical and methodological contribution of this thesis is thus my attempt to utilize highly theoretical concepts of affect theory to develop a more practically applicable feminist internet politics. In other words, I have analyzed cases in which affectivity plays a major role, and extracted tools from them that I see as relevant for feminist digital politics and activism.

All in all, what this thesis suggests is that digital intimacy, even though it is already omnipresent in everyday digital cultures, can be adopted, applied, and used strategically after careful analytical consideration. I also argue that for this purpose it is crucial to use an expanded, non-normative understanding of intimacy. I argue that the benefit of such an approach is the breaching of particular boundaries, such as digital/non-digital, public/private, embodied/disembodied, or attached/detached, categories whose dissolution can open up new 
paths for subversive digital subjectivities, or at least less stereotypical subject positions.

Taking a situated snapshot means having a limited location and partial perspective (Haraway 1991, 190), which means acknowledging that the snapshot of this cultural assemblage of digital activist approaches (hatr), the momentum of women*-centered coding initiatives and the (potentially short-lived) internet hype centered around intimate intensities can only depict a frozen moment in time. Yet, evoking Haraway's appeal for a theory and practice of objectivity that privileges "contestation, deconstruction, passionate construction, webbed connections, and hope for transformation of systems of knowledge and ways of seeing" $(1991,191)$, I have argued for the importance of understanding digital inimicalities and their webbed connections in order to find ways of doing new media differently.

Finally, turning a critical eye on the concept of digital intimacy, it has to be pointed out that such intimacy, as I have laid it out in the course of this work, is a lot about boundary confusion: boundaries between public and private, digital and non-digital, human and machine, and so on. What can be criticized is the implicated idea that boundary confusion is always subversive or somehow necessarily a 'better' state of things. In this context, Donna Haraway makes "an argument for pleasure in the confusion of boundaries and for responsibility in their construction" (Haraway 1991, 150, emphasis in original). In other words, this thesis takes the position that boundary confusion or erosion of borders is mostly a 'good' thing, particularly for an intimate digital feminist politics, insofar as it points out that assumed separations were never hermetically sealed in the first place but always were in a state of movement. New media genres, for example, do not emerge from a vacuum but rely on previous genre conventions. In the same way, conventions of gender, without having any kind of original status, iteratively find new forms in different settings (Butler 1990). That means that whilst media genres and genders rely on preexisting ways of doing them, they still add some kind of difference and entail a forms of breakage within new layers of sense-making. 
However, this blurring of boundaries has to be discussed critically and responsibly with respect to my strategic examples. For instance, while there is little doubt that the opening up of intimacy, that is, disentangling it from its institutionalization in such things as heteronormative conceptions of marriage and kinship, has done a lot for women's emancipatory projects (Giddens 1992), my empirical material shows that this is not always the case in contemporary contexts of digital intimacies.

The boundary confusions between work and life, for example, give rise here to forms of affective labor which confuse intimate life in ways that can be harmful or detrimental to the search for novel subject positioning and embodiments within digital arenas. And my material shows that this is happening alongside gendered norms; for example, when the initiatives are perceived as or actually resemble additional, unpaid, and under-acknowledged 'services with a smile', underscoring a traditional discursive connections of women with care, sociability, softness, and so on.

\section{Future Research}

It is difficult, indeed almost impossible, to predict how digital media will continue to shape our everyday lives. They are transforming at accelerating speed and, consequently, according to Jonathan Crary, there is only one thing that is certain in the context of digitalization: the "intensifying integration of one's time and activity into the parameters of electronic exchange" (Crary 2014, 40). From my point of view, this means that digital media will inevitably creep into more and more aspects of intimacy, and that we will have to learn to deal with it. As Joanne Garde-Hansen and Kristyn Gorton put it: "It is the ability to be 'emotionally competent', however detached and alienated this is, that remains a sign of one's ability to thrive in a capitalist economy. One has to be competent in emotions in new and digitally literate ways" (GardeHansen and Gorton 2013, 50). From a more decidedly affective perspective, this means not only getting a grip on emotional management 
(which is a strategy of 'detachment', as Garde-Hansen and Gorton suggest), but also finding ways of understanding and acknowledging affective attachments to digital technologies that do not necessarily fall into the realm of emotions, may it be affects of desire, memory, anger, political passion, or investments of labor and time (Hillis, Paasonen, and Petit 2015, 1).

Particularly in relation to digital inimicalities, as I have discussed them in this thesis, novel strategies are needed. We are only beginning to approach this subject matter, and I believe that it will remain an important topic for years to come. One discussion of particular importance concerns the use of digital affective tools in political ways (cf.Smith-Prei and Stehle 2016; Karatzogianni and Kuntsman 2012; Ahmed 2004), and I see my work as an attempt to contribute in this respect.

For example, while the hatr.org homepage currently seems to be dormant or infrequently updated (the most current entry is dated 11/01/2016), ${ }^{125}$ other projects designed to counteract hate speech and harassment, or to help persons affected by online hate, have surfaced recently.

One example is heartmob.com ${ }^{126}$, which, under the slogan "Join the movement to end online harassment", connects the receivers of hate messages or other forms of harassment with volunteers from all over the world who give them advice, help to document and report abuse, or simply offer consolation. ${ }^{127}$ Other initiatives that work in a similar vein, but with differing strategies, are the Hate Speech Watch of the European Council's No Hate Speech Movement ${ }^{128}$ and the rather

$125 \mathrm{http} / / /$ hatr.org/, last accessed 27-10-2016.

$126 \mathrm{https}$ ://iheartmob.org/, last accessed 27-10-2016.

127 This initiative was brought to life by the Hollaback! movement in 2013. Anybody can become a volunteer after a background check (in my understanding, of their social media accounts). An example of the kind of cases one might be confronted with as a volunteer is given on the homepage, where a tweet is displayed that reads: "An unauthorized nude image of me has been tweeted by a known harasser and Twitter has refused to act."

$128 \mathrm{http}: / /$ www.nohatespeechmovement.org/, last accessed 27-10-2016. On the homepage, it says: "Hate Speech Watch is a user-generated repository to trace, share and discuss online hate speech content. Instances of hate speech 
humorous Zerotrollerance project, which scans Twitter for offensive, sexist language and feeds the tweets to a team of voluntary 'coaches' who encourage the senders to enroll onto a six-step program that leads them "through an intensive process of self excavation", providing "practical tools to overcome their inner hurdles" in order to eventually become 'reformed'. ${ }^{129}$ From the vantage point of this thesis, it would be interesting to analyze and compare more digital initiatives like these and extract even more digital strategies for political actions against inimicality in the form of hate speech and online harassment from them.

Similarly, it would be valuable to evaluate the various coding courses' strategies and their applicability; for example, to the Swedish or European context (most of the initiatives discussed are located in the USA). As I have established, the drive to encourage women into tech can be seen as a broad societal trend that currently has significant momentum, and in recent years innumerable DIY coding groups, meetups, and networks have been initiated. In other words, today many such initiatives exist, which have more of a 'grassroots' appeal to them, and which I have not taken into account in this study. For my purpose, it is also interesting that many of them have queerfeminist orientations (Toupin 2015). Thus, a thought-provoking question for further research might be how these initiatives differ from the ones discussed here, how they negotiate digital intimacy, and the subject positions available vis-à-vis the digital.

Lastly, to date, ASMR has been an under-researched phenomenon altogether but is gradually being picked up as a subject of academic inquiry, particularly from a neuroscientific perspective, mainly in order to find out what the autonomous sensory meridian response 'is' in the first place, particularly the physical 'tickle' response (cf. Barratt and Davis 2015). However, from my vantage point of studying the cultural

signaled here are not reported to judicial authorities, regulatory bodies or internet providers. Please, also report systematically all hate speech you encounter online to the relevant national authorities and Internet companies." http://www.nohatespeechmovement.org/hate-speech-watch, last accessed 27-10-2016.

129 http://zerotrollerance.guru/index.php, last accessed 27-10-2016. 
meanings and subject positions of self-identified female performers in ASMR, another question for further research could be concerned with their long-term impact on this emerging digital genre. I am thinking in particular about the novel, partially DIY cinematic tricks or the ways in which they play with perspectives and out-of-the-ordinary narratives.

As this thesis has focused on the starlets of the scene, it could also be worth pursuing an investigation of the (few) queer ASMR performances or those that reproduce images of masculinity, separately or in comparison, also as so little is known about this subcultural phenomenon at large. Are their performances composed differently, and if so how? What elements of ASMR, as I have discussed them, remain? Which ones are transformed, if the performance of straight femininity is no longer central, or when it becomes queered? And what would this do to the logic of the male gaze (Mulvey 1989)?

In any case, the dimensions of digital intimacies, their impact on contemporary everyday cultures, and how the digital, the intimate, and people's everyday lives intermingle, leave a lot to explore. If rethinking intimacy means rethinking "how we might imagine lives that make more sense than the ones so many are living" (Berlant 2000, 6) there is no reason why this should not also include the realm of the digital. 


\section{References}

Abbate, Janet. 2010. "The Pleasure Paradox: Bridging the Gap Between Popular Images of Computing and Women's Historical Experiences." In Gender Codes: Why Women are Leaving Computing, edited by Thomas J. Misa, 213-27. Hoboken, New Jersey: Wiley; IEEE Computer Society.

Abbruzzese, Jason. 2015. "All the Feels: How a Bunch of YouTubers Discovered a Tingling Sensation Nobody Knew Existed.” Accessed December 02, 2015. http://mashable.com/2015/01/26/asmryoutube/\#exNHFo.tOkqt.

Abel, Marco. 2007. Violent Affect: Literature, Cinema, and Critique after Representation. Lincoln: University of Nebraska Press.

-—-. 2008. "Intensifying Affect." Accessed October 24, 2015. http://www.electronicbookreview.com/thread/fictionspresent/immersed.

Adam, Alison. 2002. "The Ethical Dimension of Cyberfeminism." In Reload: Rethinking women + cyberculture, edited by Mary Flanagan and Austin Booth, 158-74. Cambridge, MA: MIT Press.

Ahmed, Sara. 2004. The Cultural Politics of Emotion. 2nd edition. New York: Routledge.

-—-. 2014. "Problems with Names." Accessed April 11, 2016. https://feministkilljoys.com/2014/o4/25/problems-with-names/.

- - . 2015. "Introduction: Sexism - A Problem with a Name." New Formations 86: 5-13.

Ahmed, Sara, and Jackie Stacey, eds. 2001. Thinking Through the Skin. London: Routledge. 
Angelone, Lauren. 2012. "A Critical Discourse Analysis of Representations of Female Doctoral Student Bloggers and Implications for Education." In Cyberfeminism 2.o, edited by Radhika Gajjala and Yeon J. Oh, 61-85. Digital formations. New York: Peter Lang.

Angerer, Marie-Luise. 2015. Desire After Affect. London: Rowman \& Littlefield International.

Austin, J. L. 1975. How to Do Things with Words. 2nd edition. Cambridge, MA: Harvard University Press.

Baer, Hester. 2014. "Redoing Feminism: Digital Activism, Body Politics, and Neoliberalism." Feminism in the Digital Age: Transnational Activism in Germany and Beyond, University of Maryland, College Park, USA, November 10. Accessed November 21, 2014. https://www.youtube.com/watch?v=-3QnMNAd6_U.

Balsamo, Anne. 1991. "Feminism and Cultural Studies." The Journal of the Midwest Modern Language Association 24 (1): 50-73.

Banissy, Michael J., and Jamie Ward. 2007. "Mirror-Touch Synesthesia is Linked with Empathy." Nature Neuroscience 10 (7): 815-16.

Barratt, Emma L., and Nick J. Davis. 2015. “Autonomous Sensory Meridian Response (ASMR): a Flow-Like Mental State.” PeerJ 3 (e851): n.p.

Baudrillard, Jean. 1994. Simulacra and Simulation. Ann Arbor: University of Michigan Press.

Baudry, Jean-Louis. 1975. "Ideological Effects of the Basic Cinematographic Apparatus." Film Quarterly 28 (2): 39-47.

Benjamin, Walter. [1936] 2010. The Work of Art in the Age of Mechanical Reproduction 41. Lexington, KY: Prism Key Press.

Bensky, Tova, and Eran Fisher. 2014. Internet and Emotions 22. New York: Routledge.

Berlant, Lauren. 1998. "Intimacy: A Special Issue.” Critical Inquiry 24 (2): 281-88. 
- - 2000. Intimacy. Chicago: University of Chicago Press.

--_. 2011. Cruel Optimism. Durham: Duke University Press.

Beyer, Jessica L. 2012. "Women's (Dis)embodied Engagements with Male-Dominated Online Communities.” In Gajjala and Oh, Cyberfeminism 2.o, 153-71.

Blackman, Lisa. 2012. Immaterial Bodies: Affect, Embodiment, $M e$ diation. Theory, culture \& society. Thousand Oaks, CA: Sage.

Blue, Violet. 2015a. The Smart Girl's Guide to Privacy: Practical tips for staying safe online. San Francisco, CA: No Starch Press.

- - . 2015b. "Women, LGBT Least Safe on Facebook, Despite 'Real Name' Policy.” Accessed October 31, 2016. https://www.engadget.com/2015/o6/25/women-lgbt-safety-facebook-policy/.

Bohlin, Rebecka. 2016. Tackla Hatet: Om Näthat och Hot - och Hur du Skyddar Dig. Stockholm: Ordfront.

Bolter, Jay D., and Richard Grusin. 1999. Remediation: Understanding New Media. Cambridge, MA: MIT Press.

boyd, danah. 2009. "A Response to Christine Hine: Question 1: Defining Project Boundaries.” In Internet Inquiry: Conversations about Method, edited by Annette N. Markham and Nancy K. Baym, 2632. Los Angeles: Sage Publications.

Braidotti, Rosi. 2002. "Identity, Subjectivity and Difference: A Critical Genealogy." In Thinking differently: A reader in European women's studies, edited by Gabriele Griffin and Rosi Braidotti, 158-80. London, New York: Zed Books.

-- . 2003. "Cyberfeminism with a Difference." In Futures of critical theory: Dreams of difference, edited by Michael Peters, Mark Olssen, and Colin Lankshear, 239-6o. Lanham: Rowman \& Littlefield.

Brown, Wendy. 2003. "Neo-liberalism and the End of Liberal Democracy." Theory \& Event 7 (1). 
Butler, Judith. 1990. Gender trouble: Feminism and the Subversion of Identity. 2nd edition. Thinking gender. New York: Routledge.

-- - 1993. Bodies that Matter: On the Discursive Limits of 'Sex'. New York: Routledge.

--—. 1997. Excitable speech: A Politics of the Performative. New York: Routledge.

Bücker, Teresa. 2014. "Gestärkte Bewegungen, Geschwächte Aktivistinnen? Das Dilemma des Onlineaktivismus." Feministische Studien 32 (1): 116-27.

Carlucci Aiello, Luigia. 2016. "The Multifaceted Impact of Ada Lovelace in the Digital Age.” Artificial Intelligence 235: 58-62.

Carstensen, Tanja. 2013. "Verhandlungen von Geschlecht und Feminismus im Web 2.o.” In Geschlechterverhältnisse und neue Öffentlichkeiten: Feministische Perspektiven, edited by Birgit Riegraf, Hanna Hacker, Heike Kahlert, Brigitte Liebig, Martina Peitz, and Rosa Reitsamer, 112-27. Münster: Westfälisches Dampfboot.

Castells, Manuel. 2010. The Rise of the Network Society. 2nd edition. The information age. Malden, MA: Blackwell.

Cheadle, Harry. 2013. "ASMR, the Good Feeling no one can Explain." Accessed November 23, 2013. http://www.vice.com/read/asmrthe-good-feeling-no-one-can-explain.

Chilcott, Lesley. 2015. CodeGirl: Filmbuff.

Clough, Patricia T. 2010. "The Affective Turn: Political Economy, Biomedia, and Bodies." In The affect theory reader, edited by Melissa Gregg and Gregory J. Seigworth, 206-25. Durham: Duke University Press.

Coleman, Felicity J. 2010. "Affect." In The Deleuze dictionary, edited by Adrian Parr, 11-13. Edinburgh: Edinburgh University Press. 
Corneliussen, Hilde G. 2016. "Making the Invisible Become Visible: Recognizing Women's Relationship with Technology.” International Journal of Gender, Science and Technology 6 (2): 210-22.

Couldry, Nick. 2008. "Mediatization or Mediation? Alternative Understandings of the Emergent Space of Digital Storytelling." New Media \& Society 10 (3): 373-91.

Crary, Jonathan. 2014. 24/7: Late Capitalism and the Ends of Sleep. London: Verso Books.

Csikszentmihalyi, Mihaly. 2008. Flow: The Psychology of Optimal Experience. Harper Perennial Modern Classics. New York: Harper and Row.

Dahl, Ulrika. 2010. "Femme on Femme: Reflections on Collaborative Methods and Queer Femme-inist Ethnography." In Queer methods and methodologies: Intersecting queer theories and social science research, edited by Kath Browne and Catherine J. Nash, 143-66. London: Ashgate Publishing, Ltd.

de Lauretis, Teresa. 1987. Technologies of Gender: Essays on Theory, Film, and Fiction. Theories of representation and difference. Bloomington: Indiana University Press.

Deleuze, Gilles. 1988. Spinoza, Practical Philosophy. San Francisco: City Lights Books.

Deleuze, Gilles, and Félix Guattari. 1988. A Thousand Plateaus: Capitalism and Schizophrenia. Minneapolis: University of Minnesota Press.

Downey, Genesis. 2012. "Guilding, Gaming, and Girls." In Gajjala and Oh, Cyberfeminism 2.o, 171-87.

Drüeke, Ricarda, and Elke Zobl, eds. 2012. Feminist Media: Participatory Spaces, Networks and Cultural Citizenship. Critical media studies. Bielefeld: transcript Verlag. 
Duggan, Maeve. 2014. "Online Harrassment. Summary of Findings.” Accessed February 04, 2016. http://www.pewinternet.org/2014/10/22/online-harassment/.

Ensmenger, Nathan. 2010. The Computer Boys Take Over: Computers, Programmers, and the Politics of Technical Expertise. Cambridge, MA: MIT Press.

Faludi, Susan. 2013. "Facebook Feminism, Like It or Not." The Baffler, 2013. Accessed October o8, 2016. http://thebaffler.com/salvos/facebook-feminism-like-it-or-not.

Fernandez, Maria, and Faith Wilding. 2002. "Situating Cyberfeminisms." In Domain errors! Cyberfeminist practices, edited by Maria Fernandez, Faith Wilding, and Michelle Wright, 17-28. Brooklyn, NY: Autonomedia.

Franklin, Sarah, Celia Lury, and Jackie Stacey, eds. 2000. Global Nature, Global Culture. Gender, theory and culture. London: Sage.

Freeman, Elizabeth, Brian Herrera, Nat Hurley, Homay King, Dana Luciano, Dana Seitler, and Patricia White. 2014. "Trigger Warnings Are Flawed.” Accessed October 30, 2016. https://www.insidehighered.com/views/2014/05/29/essay-faculty-members-.

Friedan, Betty. [1963] 2001. The Feminine Mystique. New York: Norton.

Gajjala, Radhika, and Yeon J. Oh, eds. 2012. Cyberfeminism 2.o. Digital formations. New York: Peter Lang.

Gallagher, Rob. An Archive of Tingles, 2015. Podcast. Accessed December 11, 2015 .

Garde-Hansen, Joanne, and Kristyn Gorton, eds. 2013. Emotion Online: Theorizing Affect on the Internet. New York: Palgrave Macmillan.

Gardiner, Becky, Mahana Mansfield, Ian Anderson, Josh Holder, Daan Louter, and Monica Ulmanu. 2016. “The Dark Side of Guard- 
ian Comments.” Accessed April 27, 2016. https://www.theguardian.com/technology/2016/apr/12/the-dark-side-of-guardian-comments.

Geertz, Clifford. 1973. The Interpretation of Culture. New York: Basic Books.

Giddens, Anthony. 1992. The Transformation of Intimacy: Sexuality, Love, and Eroticism in Modern Societies. Stanford, CA: Stanford University Press.

Gillis, Stacy. 2004. "Neither Cyborg Nor Goddess: The (Im)Possibilities of Cyberfeminism." In Third wave feminism: A critical exploration, edited by Stacy Gillis, 185-96. Basingstoke: Palgrave Macmillan.

Glott, Ruediger, and Rishab Ghosh. 2010. "Analysis of Wikipedia Survey Data. Topic: Age and Gender differences.” Accessed May 02, 2011. http://www.wikipediasurvey.org/docs/Wikipedia_Age_Gender_30March\%202010-FINAL-3.pdf.

Goldberg, Greg. 2012. "Negotiating Affect in Media/Cultural Studies." WSQ: Women's Studies Quarterly 40 (1-2): 242-50.

Gondry, Michel. 2004. Eternal sunshine of the spotless mind. 1st ed. A Newmarket shooting script series book: Focus Features.

Graff, Bernd. 2013. "Der Gesang Flüsternder Sirenen.” Süddeutsche Zeitung, June 15. Accessed December 12, 2015.

http://www.sueddeutsche.de/kultur/video-trend-asmr-der-gesang-fluesternder-sirenen-1.1683306.

Gregg, Melissa. 2006. Cultural Studies' Affective Voices. Basingstoke, UK: Palgrave Macmillan.

---. 2011. Work's Intimacy. Cambridge, UK, Malden, MA: Polity.

Gross, Doug. 2013. "Under Pressure, Facebook Targets Sexist Hate Speech.” Accessed October 16, 2016. http://edition.cnn.com/2013/05/29/tech/social-media/facebook-hatespeech-women/index.html. 
Halberstam, Judith. 2011. The Queer art of Failure. Durham: Duke University Press.

Halpin, Harry. 2013. "Becoming Digital: Reconciling Theories of Digital Representation and Embodiment." In Philosophy and theory of artificial intelligence, edited by Vincent C. Müller, 199-215 5. Heidelberg, New York: Springer.

Hansen, Mark. 2004. New Philosophy for New Media. Cambridge, Mass: MIT Press.

Haraway, Donna J. 1990. "A Manifesto for Cyborgs: Science, Technology, and Socialist Feminism in the 1980s." In Feminism, edited by Linda J. Nicholson, 190-223. Thinking gender. New York: Routledge.

-- . 1991. Simians, Cyborgs, and Women: The Reinvention of Nature. New York: Routledge.

-—-. 1997. "The Virtual Speculum." Feminist Review 55 (1): 22-72.

Harding, Sandra G. 1986. The Science Question in Feminism. Ithaca: Cornell University Press.

---, ed. 2004. The Feminist Standpoint Theory Reader: Intellectual and Political Controversies. New York: Routledge.

Hardt, Michael. 1999. “Affective Labor.” boundary 26 (2): 89-100.

Hardt, Michael, and Antonio Negri. 2005. Multitude: War and Democracy in the Age of Empire. New York: Penguin Books.

Hartley, John, Martin Montgomery, Ellie Rennie, and Marc Brennan, eds. 2002. Communication, Cultural and Media Studies: The key concepts. 3rd ed. Routledge key guides. London: Routledge.

Hayles, N. K. 1999. How We Became Posthuman: Virtual Bodies in Cybernetics, Literature, and Informatics. Chicago, IL: University of Chicago Press.

Hebdige, Dick. 1979. Subculture, the Meaning of Style. New accents. London: Methuen. 
Herbold, Astrid. 2011. "Sag doch auch mal was." Accessed April 14, 2015. http://www.zeit.de/digital/internet/2011-02/internetfrauen-maenner.

Herring, Susan C. 1996a. "Posting in a Different Voice: Gender and Ethics in CMC.” In Philosophical perspectives on computer-mediated communication, edited by Charles Ess, 115-45. SUNY series in computer-mediated communication. Albany: State University of New York Press.

---, ed. 1996b. Computer-Mediated Communication: Linguistic, Social, and Cross-Cultural Perspectives. Amsterdam, Philadelphia: John Benjamins.

Hess, Amanda. 2014. "Why Women Aren't Welcome on the Internet." The Pacific Standard, June 1. Accessed April 26, 2016. https://psmag.com/why-women-aren-t-welcome-on-the-internetaa21fdbc8d6\#.590j1zydo.

Hillis, Ken, Susanna Paasonen, and Michael Petit, eds. 2015. Networked Affect. Cambridge, MA: MIT Press.

Hine, Christine. 2000. Virtual Ethnography. London, Thousand Oaks, CA: Sage.

Hjorth, Larissa, and Sun S. Lim. 2012. "Mobile Intimacy in an Age of Affective Mobile Media.” Feminist Media Studies 12 (4): 477-84.

Hockridge, Stephanie. 2013. "The Whisper Movement: Calming or Creepy?” ABC15 Arizona. May 16. Accessed March 10, 2014. http://www.abc15.com/news/local-news/water-cooler/asmr-whisper-therapy-does-it-work-relaxing-healing-with-sounds-and-awhisper.

hooks, bell. 2013. Writing beyond Race: Living Theory and Practice. 1st ed. New York, London: Routledge.

Hudelson, Joshua. 2012. "Listening to Whisperers: Perfomance, ASMR Community and Fetish on YouTube.” Accessed December 
10, 2015. http://soundstudiesblog.com/2012/12/10/whisper-community/.

Irigaray, Luce. 2004. Luce Irigaray: Key writings. London, New York: Continuum.

Isaacson, Walter. 2014. "The Women of ENIAC." Fortune 170: 16065.

Jansson, André. 2013. "Mediatization and Social Space: Reconstructing Mediatization for the Transmedia Age." Communication Theory (Communication Theory) 23 (3): 279-96.

Jay, Martin. 1993. Downcast Eyes: The Denigration of Vision in Twentieth-Century French Thought. Berkeley: University of California Press.

Jimroglou, Krissi M. 1999. "A Camera with a View: JenniCAM, Visual Representation, and Cyborg Subjectivity." Information, Communication \& Society 2 (4): 439-53.

Johnston, Angus. 2014. "Why I'll Add a Trigger Warning." Accessed October 30, 2016. https://www.insidehighered.com/views/2014/05/29/essay-why-professor-adding-triggerwarning-his-syllabus\#sthash.3AiFHg3f.dpbs.

Jurgenson, Nathan. 2011. "Digital Dualism versus Augmented Reality.” Accessed March 06, 2014. http://thesocietypages.org/cyborgology/2011/o2/24/digital-dualism-versus-augmented-reality/.

Kaplan, Andreas M., and Michael Haenlein. 2011. "The early bird catches the news: Nine things you should know about micro-blogging." Business Horizons 54 (2): 105-13.

Kaplan, E. A. 1987. Rocking around the Clock: Music Television, Postmodernism, and Consumer Culture. New York: Methuen.

Karatzogianni, Athina, and Adi Kuntsman, eds. 2012. Digital Cultures and the Politics of Emotion: Feelings, Affect and Technological Change. Basingstoke: Palgrave Macmillan. 
Kaun, Anne, and Karin Fast, eds. 2014. Mediatization of Culture and Everyday Life. Karlstad University Studies 13. Huddinge: Södertörns högskola.

Kember, Sarah. 2003. Cyberfeminism and Artificial Life. London: Routledge.

Kember, Sarah, and Joanna Zylinska. 2015. Life after new Media: Mediation as a Vital Process. Cambridge: MIT Press.

Kendall, Lori. 2002. Hanging out in the Virtual Pub: Masculinities and Relationships Online. Berkeley: University of California Press.

---. 2011. "'White and Nerdy': Computers, Race, and the Nerd Stereotype." Journal of Popular Culture 44 (3): 505-24.

Kenney, Moira. 2001. Mapping Gay L.A: The Intersection of Place and Politics. American subjects. Philadelphia: Temple University Press.

Kincheloe, J. L. 2001. "Describing the Bricolage: Conceptualizing a New Rigor in Qualitative Research.” Qualitative Inquiry 7 (6): 679-92.

Knappe, H., and S. Lang. 2014. "Between Whisper and Voice: Online Women's Movement Outreach in the UK and Germany." European Journal of Women's Studies 21 (4): 361-81.

Kubik, Erica. 2012. "Masteres of Technology: Defining and Theorizing the Hardcore/Casual Dichotomy in Video Game Culture." In Gajjala and Oh, Cyberfeminism 2.o, 135-53.

Kyrölä, Katariina. 2015. "Toward a Contextual Pedagogy of Pain: Trigger Warnings and the Value of Sometimes Feeling Really, Really Bad." lambda nordica 1: 126-44.

Lagesen, Vivian A. 2008. "A Cyberfeminist Utopia? Perceptions of Gender and Computer Science among Malaysian Women Computer Science Students and Faculty.” Science, Technology \& Human Values 33 (1): 5-27. 
Lasén, Amparo, and Elena Casado. 2012. "Mobile Telephony and the Remediation of Couple Intimacy." Feminist Media Studies 12 (4): 550-59.

Levina, Marina. 2012. "Our Data, Ourselves: Feminist Narratives of Empowerment in Health 2.o Discourse.” In Gajjala and Oh, Cyberfeminism 2.0, 13-29.

Lewinsky, Monica. 2015. "The Price of Shame.” TED talks. March 2015. Accessed April 30, 2016. http://www.ted.com/talks/monica_lewinsky_the_price_of_shame.

Lister, Martin, Jon Dovey, Seth Giddings, Iain Grant, and Kieran Kelly, eds. 2003. New Media: A Critical Introduction. London, New York: Routledge.

Lloyd, Genevieve. 1996. Routledge Philosophy Guidebook to Spinoza and the Ethics. London, New York: Routledge.

Lochte, Bryson C. 2013. "Touched Through a Screen: Putative Neural Correlates of Autonomous Sensory Meridian Response.” Senior honors thesis, Dartmouth College.

Luhmann, Niklas. 1986. Love as Passion: The Codification of Intimacy. Cambridge, MA: Harvard University Press.

Lykke, Nina. 2010. Feminist Studies: A Guide to Intersectional Theory, Methodology and Writing 1. London: Routledge.

Markham, Annette N. 2013. "Remix Cultures, Remix Methods: Reframing Qualitative Inquiry for Social Media Contents." In Global dimensions of qualitative inquiry, edited by Norman K. Denzin and Michael D. Giardina, 63-81. International Congress of Qualitative Inquiry 8.

Markham, Annette N., and Nancy K. Baym, eds. 2009. Internet Inquiry: Conversations about Method. Los Angeles: Sage Publications.

Marks, Laura U. 2002. Touch: Sensuous Theory and Multisensory Media. Minneapolis: University of Minnesota Press. 
McLuhan, Marshall. [1964] 2001. Understanding Media: The Extensions of Man. Routledge classics. London: Routledge.

Merica, Dan. 2014. "Lewinsky Makes Emotional Plea to end Cyberbullying." Accessed April 26, 2016. http://edition.cnn.com/2014/10/20/politics/lewinsky-cyber-bullying/index.html.

Meßmer, Anna-Katharina, Marieanne Schmidbaur, and Paula-Irene Villa. 2014. "Intimitäten - Wie Politisch ist das Vertraute?" Feministische Studien 1: 3-8. Accessed October 07, 2015.

Misa, Thomas J. 2015. "Charles Babbage, Ada Lovelace and the Bernoulli Numbers.” In Ada's legacy, edited by Robin Hammerman and Andrew L. Russell. 1st edition, 11-32.

-- , ed. 2010. Gender Codes: Why Women are Leaving Computing. Hoboken, New Jersey: Wiley; IEEE Computer Society.

Mulvey, Laura. 1989. Visual and other Pleasures. 2nd ed. Language, discourse, society. Houndmills, Basingstoke, Hampshire: Macmillan.

Nakamura, Lisa. 2002. Cybertypes: Race, ethnicity, and identity on the Internet. New York: Routledge.

National Network to End Domestic Violence. 2014. A Glimpse From the Field: How Abusers Are Misusing Technology. Washington. Accessed June 17, 2016.

Neubauer, Antonia. 2012. "Programming for Women: The Rails Girls.” Sister Magazine. Journal for the Digital Lady, October 2012.

Norris, Pippa. 2001. Digital Divide: Civic Engagement, Information Poverty, and the Internet Worldwide. Communication, society, and politics. New York: Cambridge University Press.

Novella, Steven. 2012. “ASMR.” Accessed February 03, 2016. http://theness.com/neurologicablog/index.php/asmr/. 
Nussbaum, Emily. 2011. "The Rebirth of the Feminist Manifesto: Come for the Lady Gaga, Stay for the Empowerment.” Accessed April 17, 2015. http://nymag.com/news/features/feminist-blogs2011-11/.

Obreja, Monica. 2012. Technology and Sexual Difference. Linköping: Department of Thematic Studies, Linköping University. Phd diss.

Paasonen, Susanna. 2011a. Carnal Resonance: Affect and Online Pornography. Cambridge, Mass: MIT Press.

--—. 2011b. "Revisiting Cyberfeminism." Communications 36 (3).

Padua, Sydney. 2015. The Thrilling Adventures of Lovelace and Babbage. New York: Pantheon Books.

Parikka, Jussi. 2015. "Ethologies of Software Art and Affect: What Can a Digital Body of Code do?” In Hillis, Paasonen, and Petit, Networked Affect, 103-17.

Pilcher, Jane, and Imelda Whelehan, eds. 2004. Fifty Key Concepts in Gender Studies. SAGE key concepts. London, Thousand Oaks, CA: Sage.

Plant, Sadie. 1997. Zeroes + Ones: Digital Women + the New Technoculture. 1st edition. New York: Doubleday.

Prince, Rosa. 2014. "Jennifer Lawrence: Stolen Nude Photographs were 'Sex Crime'.” The Telegraph, October 7. Accessed October 27, 2016. http://www.telegraph.co.uk/news/worldnews/northamerica/usa/11146476/Jennifer-Lawrence-stolen-nude-photographswere-sex-crime.html.

Ramachandran, V. S., and E. L. Altschuler. 2009. “The Use of Visual Feedback, in particular Mirror Visual Feedback, in Restoring Brain Function.” Brain 132 (7): 1693-1710.

Reinsberg, Alexandra. 2013. "Initiative gegen den Digitalen Mob.” Accessed April 19, 2015. http://www.fr-online.de/digital/hatr-orgsammelt-kommentare-von-trolls-initiative-gegen-den-digitalenmob,1472406,21542020.html. 
Reynolds, Robin. 2015. Cqde - Debugging the Gender Gap: Finish Line Features.

Riesewieck, Moritz. 2016. "Commercial Content Moderation - Die Müllabfuhr im Internet!”, Berlin, February 6. Accessed November o6, 2016. https://re-publica.de/16/session/commercial-contentmoderation-mullabfuhr-im-internet.

Rizzolatti, G., and M. Fabbri-Destro. 2010. "Mirror Neurons: From Discovery to Autism." Experimental Brain Research, 223-37.

Ronson, Jon. 2016. "Monica Lewinsky: 'The shame Sticks to you like Tar'.” The Guardian, April 22. Accessed October 31, 2016.

Sadowski, Helga. 2015. "From \#Aufschrei to Hatr.org: Digital-Material Entanglements in the Context of German Digital Feminist Activisms." Feminist Media Studies, 55-69.

Sandberg, Sheryl. 2015. Lean in: Women, Work, and the Will to Lead. New York: Alfred A. Knopf.

Sanjek, Roger. 1990. “A Vocabulary for Fieldnotes.” In Fieldnotes: The Makings of Anthropology, edited by Roger Sanjek. Ithaca: Cornell University Press.

Sassen, Saskia. 1998. Globalization and its Discontents: Essays on the New Mobility of People and Money. New York: New Press.

- - . 2002. "Towards a Sociology of Information Technology." Current Sociology 50 (3): 365-88.

Schaefer, Donovan. 2013. "The Promise of Affect: The Politics of the Event in Ahmed's The Promise of Happiness and Berlant's Cruel Optimism." Theory \& Event 16 (2).

Schaefer, Donovan O. 2015. Religious Affects: Animality, Evolution, and Power.

Scharff, Christina. 2014. "Christina Scharff on Gender and Neoliberalism.” Accessed August 01, 2016. http://www.theoryculturesociety.org/christina-scharff-on-gender-and-neoliberalism/. 
Schimmel, Claudia. 1998. "Der Sozialistische Bruderkuss.” Berliner Osteuropa Info 11: 81-84.

Sedgwick, Eve K., and Adam Frank. 2003. Touching Feeling: Affect, Pedagogy, Performativity. Durham: Duke University Press.

Shariatmadari, David. 2015. "Swarms, Floods and Marauders: the Toxic Metaphors of the Migration Debate." The Guardian, October 8. Accessed May 20, 2016. http://www.theguardian.com/commentisfree/2015/aug/10/migration-debate-metaphors-swarmsfloods-marauders-migrants.

Shephard, Nicole. 2013. "What was/is Cyberfeminism? Part 1 of 2." Accessed December 31, 2014. http://blogs.lse.ac.uk/gender/2013/o6/o3/what-wasis-cyberfeminism-part-1-of-2/.

Shetterly, Margot L. 2016. Hidden Figures: The American Dream and the Untold Story of the Black Women Mathematicians Who Helped Win the Space Race. New York: William Morrow.

Shildrick, Margrit. 1997. Leaky Bodies and Boundaries: Feminism, Postmodernism and (Bio)Ethics. London, New York: Routledge.

Sihvonen, Tanja, and Milla Tiainen. 2014. "I Am Gonna Stay Tonight with You' Maria's GentleWhispering: Voice, Affect and Gendered Digital Work." In Digital Labor, Sex Work, \& Maria's GentleWhispering. Conference Digital Labor: Sweatshops, Picket Lines, Barricades. November 14-16, 2014. New York.

Smart, Carol. 2006. "Intimacy." In The Cambridge Dictionary of Sociology, edited by Bryan S. Turner, 308. Cambridge England, New York: Cambridge University Press.

Smelik, Anneke. 1995. "What Meets the Eye: Feminist Film Studies." In Women's studies and culture: A feminist introduction to the humanities, edited by Rosemarie Buikema and Anneke Smelik, 6681. Atlantic Highlands, N.J. Zed Books.

Smith-Prei, Carrie. 2014. "Awkward Activism: Aesthetics and Ethics." Feminism in the Digital Age: Transnational Activism in Germany 
and Beyond, University of Alberta, November 21. Accessed November 25, 2014. https://www.youtube.com/watch?v=LcaDafyRxv4.

Smith-Prei, Carrie, and Maria Stehle. 2016. Awkward Politics: Technologies of Popfeminist Activism. Montreal and Kingston: McGillQueen's University Press.

Sobchack, Vivian C. 1992. The Address of the Eye: A Phenomenology of Film Experience. Princeton, N.J. Princeton University Press.

Spinoza, Benedictus de, Stuart Hampshire, and E. M. Curley. [1677] 1996. Ethics. London, New York: Penguin Books.

Spivak, Gayatri C. 1990. The Post-Colonial Critic: Interviews, Strategies, Dialogues. New York: Routledge.

Stafford, Tom. 2013. "The 'Unnamed Feeling' Named ASMR.” Accessed February 04, 2016. http://mindhacks.com/2013/05/13/theunnamed-feeling-named-asmr/.

Stehle, Maria. 2014. “Awkward Activism: A Methodological Starting Point." Feminism in the Digital Age: Transnational Activism in Germany and Beyond, University of Tennessee, Knoxville, USA, November 17. Accessed November 24, 2014. https://www.youtube.com/watch?v=AAQXRuqeNXQ.

Stempfhuber, Martin. 2014. "Limited Intimacy? Die Mediale Herstellung von Intimität am Beispiel von Grindr.” Feministische Studien 1: 49-62.

Storey, John. 1996. What is Cultural Studies? A Reader. London, New York: Arnold.

Straube, Wibke. 2014. Trans Cinema and its Exit Scapes: A transfeminist reading of utopian sensibility and gender dissidence in contemporary film. Linköping: Linköping University, Department of Thematic Studies. PhD Diss.

Sundén, Jenny. 2014. "Steampunk Practices: Time, Tactility, and a Racial Politics of Touch.” Ada (5). 
- - . 2015a. "Technologies of Feeling." In Hillis, Paasonen, and Petit, Networked Affect.

- - . 2015b. "Temporalities of Transition: Trans- temporal Femininity in a Human Musical Automaton.” Somatechnics 5 (2): 197-216.

Sundén, Jenny, and Malin Sveningsson. 2012. Gender and Sexuality in Online Game Cultures: Passionate Play. Routledge advances in feminist studies and intersectionality 8. New York: Routledge.

Terranova, Tiziana. 2000. "Free Labor: Producing Culture for the Digital Economy.” Social Text 18 (2): 33-58.

Theweleit, Klaus. 1987. Male Fantasies. Minneapolis: University of Minnesota Press.

Thomson, Clive. 2008. “Brave New World of Digital Intimacy.” New York Times, September 7.

Thornham, Sue. 2000. Feminist Theory and Cultural Studies: Stories of Unsettled Relations. London: Arnold.

Thornton, Sarah. 1995. Club Cultures: Music, Media and Subcultural Capital. Cambridge, UK: Polity Press.

Toupin, Sophie. 2015. "Feminist Hackerspaces as Safer Spaces?”.dpi Feminist Journal of Art and Digital Culture, n.p. Accessed August 03, 2016.

Tung, Liam. 2016. "Twitter Beefs up Troll Blocking - but its Chairman Says it must Remain Forum for 'All Voices'.” Accessed October 16, 2016. http://www.zdnet.com/article/twitter-beefs-up-troll-blocking-but-its-chairman-says-it-must-remain-forum-for-all-voices/.

Turkle, Sherry. [1984] 2005. The Second Self. 20th. anniversary ed. Cambridge (Mass.), London: MIT Press.

-- . 1988. "Computational Reticence: Why Women Fear the Intimate Machine.” In Technology and women's voices: Keeping in touch, edited by Cheris Kramarae, 33-49. New York, London: Routledge \& Kegan Paul. 
---. 2011. Alone Together: Why We Expect more from Technology and Less from Each Other. New York: Basic Books.

Turner, Bryan S., ed. 2006. The Cambridge Dictionary of Sociology. Cambridge England, New York: Cambridge University Press.

Vagianos, Alanna. 2014. “This Is What It's Like To Be A Woman Online.” Accessed November 05, 2016. http://www.huffingtonpost.com/2014/o9/19/what-its-like-to-be-a-woman-onlinebuzzfeed_n_5849052.html.

Wajcman, Judy. 2001. "Gender and Technology." In International Encyclopedia of the Social \& Behavioral Sciences, edited by Neil J. Smelser and Paul B. Baltes. 1st ed, 5976-79. Amsterdam, New York: Elsevier.

-- . 2010. "Feminist Theories of Technology." Cambridge Journal of Economics 34 (1): 143-52.

van den Boomen, Marianne, Sybille Lammens, Ann-Sophie Lehmann, Joost Raessens, and Mirko T. Schäfer, eds. 2009. Digital Material: Tracing New Media in Everyday Life and Technology. MediaMatters. Amsterdam: Amsterdam University Press.

White, Michele. 2003. "Too Close to See: Men, Women and Webcams." New Media \& Society 5 (1): 7-28.

Wilson, Ara. 2016. “The Infrastructure of Intimacy." Signs: Journal of Women in Culture and Society 41 (2): 247-80.

Åsberg, Cecilia, and Nina Lykke. 2010. "Feminist Technoscience Studies.” European Journal of Women's Studies 17 (4): 299-305. 